Pacific

Journal of

Mathematics

PARAMETRIZATION OF THE IMAGE OF NORMALIZED INTERTWINING OPERATORS

Chris Jantzen and Henry H. Kim 


\title{
PARAMETRIZATION OF THE IMAGE OF NORMALIZED INTERTWINING OPERATORS
}

\author{
Chris Jantzen and Henry H. Kim
}

\begin{abstract}
In studying residual automorphic representations, we need to parametrize the image of normalized local intertwining operators. This has been done by Moeglin in the case of the residual spectrum attached to the trivial character of the torus for split classical groups. In this paper, we extend her result to non-trivial characters of the torus. To do this, we use Roche's Hecke algebra isomorphisms and Barbasch-Moy's graded algebra isomorphisms to reduce to the case of the trivial character. Along the way, we need to show that Roche's Hecke algebra isomorphisms are compatible with induction in stages, construct a generalized Iwahori-Matsumoto involution, and show that the images of intertwining operators behave well with respect to the Hecke algebra and graded algebra isomorphisms. We note that this also gives a parameterization of the square-integrable and tempered representations supported on the Borel subgroup.
\end{abstract}

\section{Introduction.}

Let $G=G(n)$ be a split classical group $S p(2 n, F), S O(2 n+1, F)$, or $O(2 n, F)$ over a $p$-adic field $F$ with odd residual characteristic (this condition comes from $[\mathbf{R}]$ ) and $T$ be a maximal torus. Throughout this paper, we will drop $F$ in the notation. Let $\chi$ be a unitary character of $T$ and $\mathfrak{p}=\left(a_{1}, b_{1}, \ldots, a_{s}, b_{s}, a_{s+1}\right)$ be a chain $\left(a_{s+1}\right.$ is only for the cases $S p(2 n, F)$ and $S O(2 n+1, F)$; see Definition 3.1). Then $\mathfrak{p}$ gives rise to a character

$$
\begin{aligned}
\lambda_{\mathfrak{p}}=\left(\frac{a_{1}-1}{2}, \frac{a_{1}-3}{2}, \ldots,-\frac{b_{1}-1}{2}\right. & , \ldots, \frac{a_{s}-1}{2}, \frac{a_{s}-3}{2}, \ldots, \\
& \left.-\frac{b_{s}-1}{2}, \frac{a_{s+1}-1}{2}, \frac{a_{s+1}-3}{2}, \ldots, 1\right) .
\end{aligned}
$$

If we ignore the ordering, $\mathfrak{p}$ gives rise to a unipotent orbit $O$ in the dual group $G^{*}=S O(2 n+1, \mathbb{C}), S p(2 n, \mathbb{C})$, or $O(2 n, \mathbb{C})$. To $\mathfrak{p}$, we attach a Weyl group element $w_{\mathfrak{p}}$. In this paper we parametrize the image of the local normalized intertwining operator $R\left(w_{\mathfrak{p}}, \lambda_{\mathfrak{p}}, \chi\right) I\left(\lambda_{\mathfrak{p}}, \chi\right)$. We need this result in 
the calculation of the residual spectrum [Ki3]. In addition to the application to the residual spectrum, our result has independent interest in that it parametrizes the square integrable representations which have support on Borel subgroups, via the generalized Iwahori-Matsumoto involution; actually it parametrizes tempered representations which have support on Borel subgroups (see Remark 3.2.4).

In a remarkable paper [M1], Møglin solved the problem in the case when $\chi$ is trivial and $\mathfrak{p}$ satisfies a certain condition, that is, $\mathfrak{p} \in P(O)$ (see Section 2). She showed that $R\left(w_{\mathfrak{p}}, \lambda_{\mathfrak{p}}\right) I\left(\lambda_{\mathfrak{p}}\right)$ is semi-simple and its summands are parametrized by certain characters $\eta$ of $A(O)$, where $A(O)$ is a finite abelian group generated by the order two elements $\sigma\left(a_{1}\right), \sigma\left(b_{1}\right), \ldots, \sigma\left(a_{s}\right), \sigma\left(b_{s}\right)$, $\sigma\left(a_{s+1}\right)$ (we take only distinct ones). If $O$ is a distinguished unipotent orbit (i.e., $a_{i}$ 's, $b_{j}$ 's are all distinct), then the characters are those which satisfy $\eta\left(\sigma\left(a_{i}\right)\right)=\eta\left(\sigma\left(b_{i}\right)\right), i=1, \ldots, s$ and $\eta\left(\sigma\left(a_{s+1}\right)\right)=1$. We denote by $\bar{A}(\mathfrak{p})$ the set of such characters. Let $\operatorname{Unip}(\mathfrak{p})$ be the set of direct summands of $R\left(w_{\mathfrak{p}}, \lambda_{\mathfrak{p}}\right) I\left(\lambda_{\mathfrak{p}}\right)$. To a unipotent orbit $O$, she considered a certain set of ordered partitions $P(O)$ so that each chain $\mathfrak{p}^{\prime} \in P(O)$ gives rise to a certain character $\lambda_{\mathfrak{p}^{\prime}}$ which is a conjugate of $\lambda_{\mathfrak{p}}$. Let $\operatorname{Unip}(O)$ be the union of all $\operatorname{Unip}(\mathfrak{p})$ as $\mathfrak{p}$ runs through $P(O)$. She showed that $\operatorname{Unip}(O)$ is the set of irreducible constituents of the principal series $I\left(\lambda_{\mathfrak{p}}\right)$ whose Iwahori-Matsumoto involution is tempered. Then $\operatorname{Unip}(O)$ is parametrized by Springer $(O)$, which is the union of $\bar{A}(\mathfrak{p})$ as $\mathfrak{p}$ runs through $P(O)$; recall that the Springer correspondence is an injective map from the characters of $W$, the Weyl group, into the set of $(O, \eta)$, where $O$ is a unipotent orbit of $G^{*}$ and $\eta$ is a character of $A(O)$. Then Springer $(O)$ is the set of characters of $A(O)$ which are in the image of the Springer correspondence. Thus Mœglin showed that $\operatorname{Unip}(O)$ is the set of the local components of the residual spectrum attached to the trivial character of the maximal torus.

The basic approach of this paper is to reduce the problem to the trivial character case. However, there are a number of non-trivial obstacles, which we now describe.

First, the basic mechanism we use to reduce from the ramified case to the unramified case is the Hecke algebra isomorphisms of Roche $[\mathbf{R}]$. The first basic problem we must deal with is that the representations we are interested in are not, in general, induced off the Borel, but rather are degenerate principal series. So, in order to implement our approach, we must establish that these isomorphisms behave well with respect to induction in stages. Since such results may be of broader use, we do this in the generality of $[\mathbf{R}]$, not just for the particular classical groups we deal with in the rest of this paper. In addition, we need to generalize the Iwahori-Matsumoto involution. (While this has been done in general in [Au1], [Au2], [Sc-St], these results are done in the Grothendieck group setting; we need to know 
that composition series are respected as well.) Such a generalized IwahoriMatsumoto involution can easily be defined using Roche's isomorphism. But in order to verify some of the properties we need, we have to establish how it behaves with respect to induction in stages. Again, this comes down to showing that Roche's isomorphisms respect induction in stages. These issues are addressed in Section 1.

Second, we need to deal with non-trivial unramified quadratic characters. Møglin depended on Barbasch-Moy's results [B-Mo1] which use KazhdanLusztig's parametrization of unramified representations and the IwahoriMatsumoto involution. However, the technique cannot be extended to nontrivial unramified quadratic characters. In a subsequent work, BarbaschMoy [B-Mo2] extended their results to non-trivial unramified quadratic characters, using graded Hecke algebras. We use their graded Hecke algebra isomorphisms to reduce to the trivial character case. See Section 3.1. Unfortunately, Barbasch-Moy's results [B-Mo2] are stated for connected groups and we need them for the disconnected group $O(2 n)$. We have stated this as Assumption 3.1.1. We have no doubt that it is true. However, we were not able to verify it. Therefore, our results are complete only for odd orthogonal groups.

Third, we need to remove a restriction on $\mathfrak{p}$ (see Remark 3.2) for an arbitrary chain when $\chi$ is trivial. Note that an arbitrary chain does not belong to $P(O)$ in general. But it always comes from the global consideration when $\chi=\chi(\underbrace{\mu_{1}, \ldots, \mu_{1}}_{r_{1}}, \ldots, \underbrace{\mu_{k}, \ldots, \mu_{k}}_{r_{k}}, \underbrace{1, \ldots, 1}_{r_{0}})$, where $\mu_{i}$ 's are non-trivial quadratic grössencharacters such that $\mu_{i v}=1$. (See Remark 3.3.) Mœglin's argument by induction shows that $R\left(w_{\mathfrak{p}}, \lambda_{\mathfrak{p}}, 1\right) I\left(\lambda_{\mathfrak{p}}\right)$ is still semi-simple in the general case and we denote the set of direct summands still by $\operatorname{Unip}(\mathfrak{p})$. However, Moeglin's argument does not work in this case, since the normalized local intertwining operators could vanish. For this, we use the global method. By considering the iterated residue of the pseudo-Eisenstein series as in Møglin $[\mathbf{M} 1]$, we can show that $\operatorname{Unip}(\mathfrak{p})$ is contained in $\operatorname{Unip}(O)$, where $O$ is the unipotent orbit obtained by ignoring the ordering in $\mathfrak{p}$. Recall that $\operatorname{Unip}(O)$ is the union of $\operatorname{Unip}(\mathfrak{p})$ as $\mathfrak{p}$ runs through $P(O)$ and this shows that by considering arbitrary chains, we do not get a new component.

Fourth, we need to show that Hecke algebra isomorphisms of $[\mathbf{R}]$ and the graded Hecke algebra isomorphisms of [B-Mo2] commute with the intertwining operators. That reduces us to the case of the trivial character. More precisely, let $\mathfrak{p}=\left(a_{1}, b_{1}, \ldots, a_{s}, b_{s}, a_{s+1}\right)$ and $\chi=\chi(\underbrace{\mu_{1}, \ldots, \mu_{1}}_{r_{1}}, \ldots$, $\underbrace{\mu_{k}, \ldots, \mu_{k}}_{r_{k}}, \underbrace{1, \ldots, 1}_{r_{0}}), r_{0}+\cdots+r_{k}=n, r_{1} \geq \cdots \geq r_{k}, \mu_{i}$ 's are distinct quadratic characters. Here $k \leq 3$. (Recall that we are dealing with a $p$-adic field 
with odd residual characteristic and hence there are only three non-trivial distinct quadratic characters.) Set $\mu_{0}=1$. Let $G^{\prime}=G_{1}^{\prime} \times \cdots \times G_{k}^{\prime} \times G_{0}^{\prime}$, where, for $i=1, \ldots, k$,

$$
G_{0}^{\prime}=G\left(r_{0}\right), \quad G_{i}^{\prime}= \begin{cases}O\left(2 r_{i}\right), & \text { if } G=S p(2 n), O(2 n) \\ S O\left(2 r_{i}+1\right), & \text { if } G=S O(2 n+1) .\end{cases}
$$

Here we note that $G^{\prime}$ is an endoscopic group of $G$. Then by the Hecke algebra isomorphisms of $[\mathbf{R}]$ and the graded algebra isomorphisms of [B-Mo2], we get an equivalence of categories

$$
\mathcal{R}\left(G, \tau\left(\lambda_{\mathfrak{p}}, \chi\right)\right) \simeq \mathcal{R}\left(G^{\prime}, \tau^{\prime}\left(\lambda_{\mathfrak{p}}, 1\right)\right)
$$

where $\tau\left(\lambda_{\mathfrak{p}}, \chi\right)$ is the infinitesimal character associated to subquotients of $I\left(\lambda_{\mathfrak{p}}, \chi\right)$ and $\mathcal{R}\left(G, \tau\left(\lambda_{\mathfrak{p}}, \chi\right)\right)$ is the category of smooth finite-length representations of $G$ having infinitesimal character $\tau\left(\lambda_{\mathfrak{p}}, \chi\right)$. We note that $\lambda_{\mathfrak{p}}$ may be viewed as a character of the maximal split torus of $G^{\prime}$ in the obvious way: If we write $\lambda_{\mathfrak{p}}=\lambda_{\mathfrak{p}_{1}} \times \cdots \times \lambda_{\mathfrak{p}_{k}} \times \lambda_{\mathfrak{p}_{0}}$ (corresponding to the decomposition $r_{1}+\cdots+r_{k}+r_{0}=n$ above), then $\lambda_{\mathfrak{p}_{i}}$ may be viewed as a character of the maximal split torus of $G_{i}^{\prime}$. We show in Section 3.2 that under the category equivalence, the image $R\left(w_{\mathfrak{p}}, \lambda_{\mathfrak{p}}, \chi\right) I\left(\lambda_{\mathfrak{p}}, \chi\right)$ corresponds to the image $R\left(w_{\mathfrak{p}_{1}}, \lambda_{\mathfrak{p}_{1}}, 1\right) I\left(\lambda_{\mathfrak{p}_{1}}\right) \otimes \cdots \otimes R\left(w_{\mathfrak{p}_{k}}, \lambda_{\mathfrak{p}_{k}}, 1\right) I\left(\lambda_{\mathfrak{p}_{k}}\right) \otimes R\left(w_{\mathfrak{p}_{0}}, \lambda_{\mathfrak{p}_{0}}, 1\right) I\left(\lambda_{\mathfrak{p}_{0}}\right)$. This reduces us to the case when $\chi=1$. Thus, by matching of the images of intertwining operators, we see that $R\left(w_{\mathfrak{p}}, \lambda_{\mathfrak{p}}, \chi\right) I\left(\lambda_{\mathfrak{p}}, \chi\right)$ is semi-simple. Let $\operatorname{Unip}(\mathfrak{p}, \chi)$ be the set of the direct summands. Then under the category equivalence, $\operatorname{Unip}(\mathfrak{p}, \chi)$ is contained in the set $\operatorname{Unip}\left(O_{1}\right) \times \cdots \times \operatorname{Unip}\left(O_{k}\right) \times$ $\operatorname{Unip}\left(O_{0}\right)$, where $O_{i}$ are certain unipotent orbits obtained by ignoring the ordering in $\mathfrak{p}$. In particular, the generalized Iwahori-Matsumoto involution of the elements in $\operatorname{Unip}(\mathfrak{p}, \chi)$ is tempered.

We let $\chi=1$ and parametrize $\operatorname{Unip}(\mathfrak{p})$. To each $\left(a_{i}, b_{i}\right)$, we can attach a Weyl group element $\sigma_{\left(a_{i}, b_{i}\right)}$. Then $\sigma_{\left(a_{1}, b_{1}\right)}$ defines a normalized operator $R\left(\sigma_{\left(a_{1}, b_{1}\right)}\right)$. It defines a homomorphism from the group $\left\{i d, \sigma_{\left(a_{1}, b_{1}\right)}\right\}$ into the group of the intertwining operators of $R\left(w_{\mathfrak{p}}, \lambda_{\mathfrak{p}}\right) I\left(\lambda_{\mathfrak{p}}\right)$. This means the following: For $X \in \operatorname{Unip}(\mathfrak{p})$, let $R\left(\sigma_{\left(a_{i}, b_{i}\right)}\right) X=\eta_{X}^{\mathfrak{p}}\left(\sigma_{\left(a_{i}, b_{i}\right)}\right) X$. Then $\eta_{X}^{\mathfrak{p}}$ defines a character of $A(O)$ such that $\eta_{X}^{\mathfrak{p}}\left(\sigma\left(a_{i}\right)\right)=\eta_{X}^{\mathfrak{p}}\left(\sigma\left(b_{i}\right)\right)$. Since $\operatorname{Unip}(\mathfrak{p}) \subset \operatorname{Unip}(O), \eta_{X}^{\mathfrak{p}} \in \operatorname{Springer}(O)$. Therefore we have:

Theorem 0.1 (Theorem 3.4.2). Unip $(\mathfrak{p})$ is parametrized by

$$
\begin{aligned}
C(\mathfrak{p})=\{\eta \in \text { Springer }(O): & \eta\left(\sigma\left(a_{i}\right)\right)= \\
& \eta\left(\sigma\left(b_{i}\right)\right), \\
& \left.i=1, \ldots, s, \quad \eta\left(\sigma\left(a_{s+1}\right)\right)=1\right\} .
\end{aligned}
$$

Note that if $\mathfrak{p}$ satisfies the condition (3.1) (that is, in Mœglin's situation), then $C(\mathfrak{p})=\bar{A}(\mathfrak{p})([\mathrm{M} 1$, Proposition 1.3.3]). In order to apply this theorem to the residual spectrum calculation, let $O_{1}, O_{2}$ be two distinguished unipotent orbits in $G_{1}^{*}, G_{2}^{*}$, resp. (If $G=S p(2 n)$, then $G_{1}^{*}=O\left(2 r_{1}, \mathbb{C}\right.$ ) 
and $G_{2}^{*}=S O\left(2 r_{0}+1, \mathbb{C}\right)$. If $G=S O(2 n+1)$, then $G_{1}^{*}=S p\left(2 r_{1}, \mathbb{C}\right)$ and $G_{2}^{*}=S p\left(2 r_{0}, \mathbb{C}\right)$. If $G=O(2 n)$, then $G_{1}^{*}=O\left(2 r_{1}, \mathbb{C}\right)$ and $G_{2}^{*}=O\left(2 r_{0}, \mathbb{C}\right)$.) Then we get a unipotent orbit $O$ in $G^{*}$ by combining $O_{1}$ and $O_{2}$. Further we have canonical embedding $\widehat{A\left(O_{i}\right)} \subset \widehat{A(O)}$. For $\mathfrak{p}_{i} \in P\left(O_{i}\right), i=1,2$, we get a chain $\mathfrak{p}_{1} \times \mathfrak{p}_{2}$ by shuffling the segments in $\mathfrak{p}_{1}$ and $\mathfrak{p}_{2}$ so that it satisfies (3.1), and thus we get $\operatorname{Unip}\left(\mathfrak{p}_{1} \times \mathfrak{p}_{2}\right)$. Let $\operatorname{Unip}\left(O_{1}, O_{2}\right)$ be the union of $\operatorname{Unip}\left(\mathfrak{p}_{1} \times \mathfrak{p}_{2}\right)$ as $\mathfrak{p}_{i}$ runs through $P\left(O_{i}\right)$ for $i=1,2$. It is a subset of $\operatorname{Unip}(O)$. Then we have:

Theorem 0.2 (Theorem 3.4.3). $\operatorname{Unip}\left(O_{1}, O_{2}\right)$ is parametrized by

$$
\begin{aligned}
C\left(O_{1}, O_{2}\right)=\{\eta \in \text { Springer }(O): & \left.\eta\right|_{A\left(O_{1}\right)} \in \text { Springer }\left(O_{1}\right), \\
& \left.\left.\eta\right|_{A\left(O_{2}\right)} \in \text { Springer }\left(O_{2}\right)\right\} .
\end{aligned}
$$

This can be easily generalized to an arbitrary character. Let us state the result on the local components of the residual spectrum attached to an arbitrary character in order to apply it to $[\mathbf{K i 3}]$. Let $\chi=\chi(\underbrace{\mu_{1}, \ldots, \mu_{1}}_{r_{1}}, \ldots$, $\underbrace{\mu_{k}, \ldots, \mu_{k}}_{r_{k}}, \underbrace{1, \ldots, 1}_{r_{0}}), r_{0}+\cdots+r_{k}=n, r_{1} \geq \cdots \geq r_{k} \geq 2, \mu_{i}$ 's are distinct non-trivial quadratic grössencharacters. Let $O_{i}$ be a distinguished unipotent orbit in $G_{i}^{*}$ for $i=0,1, \ldots, k$, where, for $i=1, \ldots, k$,

$$
\begin{aligned}
& G_{i}^{*}= \begin{cases}O\left(2 r_{i}, \mathbb{C}\right), & \text { if } G=S p(2 n), O(2 n) \\
S p\left(2 r_{i}, \mathbb{C}\right), & \text { if } G=S O(2 n+1),\end{cases} \\
& G_{0}^{*}= \begin{cases}S O\left(2 r_{0}+1, \mathbb{C}\right), & \text { if } G=S p(2 n) \\
S p\left(2 r_{0}, \mathbb{C}\right), & \text { if } G=S O(2 n+1) \\
O\left(2 r_{0}, \mathbb{C}\right), & \text { if } G=O(2 n) .\end{cases}
\end{aligned}
$$

Let $\mathfrak{p}_{i} \in P\left(O_{i}\right)$ for $i=0, \ldots, k$ and $\mathfrak{p}=\mathfrak{p}_{1} \times \cdots \times \mathfrak{p}_{k} \times \mathfrak{p}_{0}$. Then we can shuffle the segments in $\mathfrak{p}$ so that it satisfies the condition (3.1). We still call it $\mathfrak{p}$. For a non-archimedean place $v$, let $\operatorname{Unip}\left(O_{1}, \ldots, O_{k}, O_{0}, \chi_{v}\right)$ be the set of union of $\operatorname{Unip}\left(\mathfrak{p}_{1}, \ldots, \mathfrak{p}_{k}, \mathfrak{p}_{0}, \chi_{v}\right)$ as $\mathfrak{p}_{i}$ runs through $P\left(O_{i}\right)$ for $i=0, \ldots, k$.

Theorem 0.3 (Theorem 3.4.10). $\Pi_{r e s_{v}}=\operatorname{Unip}\left(O_{1}, \ldots, O_{k}, O_{0}, \chi_{v}\right)$ is parametrized by

$C\left(O_{1}, \ldots, O_{k}, O_{0}, \chi_{v}\right)=\left[\operatorname{Springer}\left(O_{1}\right) \times \cdots \times\right.$ Springer $\left.\left(O_{k}\right) \times \operatorname{Springer}\left(O_{0}\right)\right]$, where [ ] is defined as follows: If $\mu_{1 v}=\mu_{2 v} \neq \mu_{i v}$ for $i=0,3, \ldots, k$, then we replace Springer $\left(O_{1}\right) \times$ Springer $\left(O_{2}\right)$ by

$C\left(O_{1}, O_{2}, \mu_{1 v}\right)=\left\{\eta \in\right.$ Springer $(O):\left.\eta\right|_{A\left(O_{i}\right)} \in \operatorname{Springer}\left(O_{i}\right)$, for $\left.i=1,2\right\}$, 
where $O$ is the unipotent orbit of $G_{12}^{*}$ obtained by combining $O_{1}, O_{2}$, where

$$
G_{12}^{*}= \begin{cases}O\left(2\left(r_{1}+r_{2}\right), \mathbb{C}\right), & \text { if } G=S p(2 n), O(2 n) \\ S p\left(2\left(r_{1}+r_{2}\right), \mathbb{C}\right), & \text { if } G=S O(2 n+1) .\end{cases}
$$

Acknowledgments. We would like to thank Prof. Shahidi for his guidance and encouragement throughout this paper. The second author would like to thank Prof. Mœglin for patiently answering many of his questions [M6] and for many correspondences. We would also like to thank Alan Roche for many useful conversations on Hecke algebra isomorphisms. Finally, we wish to thank Professors A. Moy, M. Reeder, A. Silberger, M. Tadić, and D. Vogan for many helpful correspondences.

\section{Hecke algebra isomorphisms and the generalized Iwahori-Matsumoto involution.}

In order to reduce from the case of ramified characters to the case of unramified characters, we use the Hecke algebra isomorphisms of Roche $[\mathbf{R}]$. We also use these isomorphisms to construct a generalized Iwahori-Matsumoto involution. (The duality results of Aubert and Schneider-Stuhler are in the Grothendieck group setting; we need to deal with the composition series here.) We begin this section by reviewing the Iwahori-Matsumoto involution and the Hecke algebra isomorphisms of Roche. We then show that these isomorphisms behave well with respect to induction in stages. This will also allow us to verify certain properties of the generalized Iwahori-Matsumoto involution.

For this section, we work in a more general setting. Let $G$ denote the $F$-rational points of a split connected reductive group defined over $F$. In order to apply Roche's results, we also assume the residue characteristic of $F$ satisfies the conditions in $[\mathbf{R}]$. For our applications to $S p(2 n), S O(2 n+$ 1 ), this requires odd residue characteristic. (We note that [Go], or more generally $[\mathbf{M r}]$, gives similar Hecke algebra isomorphisms which could be used to extend the results of this section to cover characters of level 0 without the constraints on the residue characteristic.) Fix a set of positive roots $\Phi^{+}$ and a subset of simple roots $\Pi$. Let

$$
\begin{aligned}
B & =\text { Borel subgroup of } G \\
I & =\text { Iwahori subgroup of } G \\
K & =\mathcal{O}-\text { points in } G \text { (a maximal compact subgroup) } \\
\bar{W} & =\text { Weyl group of } G \\
W & =\text { affine Weyl group of } G \\
\ell(\cdot) & =\text { length function on } W \\
T & =\text { maximal split torus in } B
\end{aligned}
$$




$$
\delta(\cdot)=\text { modular function on } B .
$$

We may view $\bar{W} \subset W$ by identifying $\bar{W}$ with $N(\mathcal{O}) / A(\mathcal{O})$, where $N=$ $\operatorname{Norm}_{G}(T)$. We use $B_{G}, I_{G}$, etc., if there is more than one group around and confusion is possible. Set

$$
\mathcal{H}(G)=C_{c}^{\infty}(G)
$$

It is an algebra under convolution. If $(\pi, G, V)$ is a smooth representation, we define $\pi$ on $\mathcal{H}(G)$ by

$$
\pi(h) v=\int_{G} h(g) \pi(g) v d g
$$

for all $h \in \mathcal{H}(G), v \in V$.

We begin by reviewing the Iwahori-Matsumoto involution. First, let us normalize Haar measure so that $|I|=1$. Set $1_{I}=\operatorname{char}_{I}$. Let

$$
\begin{aligned}
\mathcal{H}\left(G, 1_{I}\right)=1_{I} \cdot \mathcal{H}(G) \cdot 1_{I}=\left\{f \in \mathcal{H}(G) \mid f\left(i_{1} g i_{2}\right)\right. & =f(g) \\
& \text { for all } \left.i_{1}, i_{2} \in I, g \in G\right\} .
\end{aligned}
$$

The Iwahori-Matsumoto involution is an involution of $\mathcal{H}\left(G, 1_{I}\right)$. In order to describe the Iwahori-Matsumoto involution, we must first discuss the structure of $\mathcal{H}\left(G, 1_{I}\right)$. The following description is due to Bernstein-Zelevinsky (cf. [Lu2]); the classical description is due to Iwahori-Matsumoto [I-M]. As a vector space, $\mathcal{H}\left(G, 1_{I}\right)=\mathcal{H}\left(K, 1_{I}\right) \otimes \Theta$. Now, $\mathcal{H}\left(K, 1_{I}\right)$ has $\left\{T_{w}\right\}_{w \in \bar{W}}$ as a basis, where $T_{w}$ denotes the characteristic function of $I w I$. Further, the multiplication is governed by

$$
\begin{gathered}
T_{s}^{2}=(q-1) T_{s}+q \text { for } s \text { simple } \\
T_{w_{1}} T_{w_{2}}=T_{w_{1} w_{2}} \text { if } \ell\left(w_{1}\right)+\ell\left(w_{2}\right)=\ell\left(w_{1} w_{2}\right) .
\end{gathered}
$$

$\Theta$ is an abelian subalgebra of $\mathcal{H}\left(G, 1_{I}\right)$ with basis $\left\{\theta_{t} \mid t \in T / T \cap K\right\}$. For $t \in$ $T$, choose $t_{1}, t_{2} \in T^{-}=\{t \in T|| \alpha(t) \mid \leq 1$ for all simple roots $\alpha\}$ such that $t=t_{1} t_{2}^{-1}$. Then, $\theta_{t}=\delta^{\frac{1}{2}}(t) T_{t_{1}} T_{t_{2}}^{-1}$. The multiplication between $\mathcal{H}\left(K, 1_{I}\right)$ and $\Theta$ is governed by the following: If $s=s_{\alpha}, \alpha$ a simple root,

$$
\theta_{t} T_{s}=T_{s} \theta_{s t s}+(q-1) \frac{\theta_{t}-\theta_{s t s}}{1-\theta_{\check{\alpha}\left(\varpi^{-1}\right)}},
$$

where $\check{\alpha}$ denotes the coroot associated to $\alpha$. The Iwahori-Matsumoto involution $j: \mathcal{H}\left(G, 1_{I}\right) \longrightarrow \mathcal{H}\left(G, 1_{I}\right)$ is defined by

$$
\begin{aligned}
& j: T_{w} \longmapsto(-q)^{\ell(w)}\left(T_{w^{-1}}\right)^{-1} \\
& j: \theta_{t} \longmapsto \theta_{t}^{-1}
\end{aligned}
$$

for $T_{w} \in \mathcal{H}\left(K, 1_{I}\right), \theta_{t} \in \Theta$. 
We now discuss Roche's results $[\mathbf{R}]$. The applications to the representation theory of $G$ will be discussed later; for now we focus on the results dealing with the structure of Hecke algebras. Fix a character $\chi: T \cap K \longrightarrow \mathbb{C}^{\times}$. To the character $\chi$, he associates an open compact subgroup $J$ and a character $\rho: J \longrightarrow \mathbb{C}^{\times}$with $\left.\rho\right|_{T \cap K}=\chi$. The pair $(J, \rho)$ is a type in the sense of $[\mathbf{B u}-\mathbf{K}]$. Let $e_{\rho}$ be defined by

$$
e_{\rho}(g)= \begin{cases}\frac{1}{|J|} \rho^{-1}(g), & \text { if } g \in J \\ 0, & \text { if not. }\end{cases}
$$

Then, $e_{\rho}^{2}=e_{\rho}$. Let

$$
\begin{aligned}
\mathcal{H}(G, \rho) & =e_{\rho} \cdot \mathcal{H}(G) \cdot e_{\rho} \\
& =\left\{f \in \mathcal{H}(G) \mid f\left(j_{1} g j_{2}\right)=\rho^{-1}\left(j_{1} j_{2}\right) f(g) \text { for all } j_{1}, j_{2} \in J, g \in G\right\} .
\end{aligned}
$$

Roche constructs a split connected reductive group $H$ and a finite abelian group $C_{\chi}$, which acts on $H$, such that

$$
\mathcal{H}(G, \rho) \cong \mathcal{H}\left(H, 1_{I}\right) \tilde{\otimes} \mathbb{C}\left[C_{\chi}\right] .
$$

The notation $\tilde{\otimes}$ is used to indicate that multiplication is governed by

$$
\left(T_{w_{1}} \otimes c_{1}\right) \cdot\left(T_{w_{2}} \otimes c_{2}\right)=T_{w_{1}} T_{c_{1}\left(w_{2}\right)} \otimes c_{1} c_{2}
$$

for $w_{1}, w_{2} \in H, c_{1}, c_{2} \in C_{\chi}$ (cf. Section $\left.8[\mathbf{R}]\right)$. He also constructs a disconnected group $\tilde{H}$, with $H$ the connected component of the identity in $\tilde{H}$, which has $\mathcal{H}\left(H, 1_{I}\right) \tilde{\otimes} \mathbb{C}\left[C_{\chi}\right] \cong \mathcal{H}\left(\tilde{H}, 1_{I_{H}}\right)$. The following example will be of interest in $\S 3.2$.

Example 1.1. Suppose $G=S O\left(2\left(r_{0}+r_{1}\right)+1, F\right)$ or $S p\left(2\left(r_{0}+r_{1}\right), F\right), F$ of odd residual characteristic. Let $\mu$ denote a ramified quadratic character of $F^{\times}$and set $\chi=\chi(\underbrace{\mu, \ldots, \mu}_{r_{1}}, \underbrace{1, \ldots, 1}_{r_{0}})$. (More precisely, it is $\left.\chi\right|_{T(\mathcal{O})}$ that is needed in Roche's construction.) Then $J_{\chi}=I$, and we have the following:

(1) If $G=S O\left(2\left(r_{0}+r_{1}\right)+1\right)$, then $\mathcal{H}\left(G, \rho_{\chi}\right) \cong \mathcal{H}(\tilde{H}, 1)$, with $\tilde{H}=$ $S O\left(2 r_{1}+1\right) \times S O\left(2 r_{0}+1\right)=H_{1}^{\prime} \times H_{0}^{\prime}$.

(2) If $G=S p\left(2\left(r_{0}+r_{1}\right)\right)$, then $\mathcal{H}\left(G, \rho_{\chi}\right) \cong \mathcal{H}(\tilde{H}, 1)$, with $\tilde{H}=O\left(2 r_{1}\right) \times$ $S p\left(2 r_{0}\right)=H_{1}^{\prime} \times H_{0}^{\prime}$.

Further, if $\lambda$ is an unramified character of $T$, write $\lambda=\lambda_{1} \times \lambda_{0}$ (with $\lambda_{1}$ the character of $\left(F^{\times}\right)^{r_{1}}$ consisting of the first $r_{1}$ terms of $\lambda$ and $\lambda_{0}$ the character of $\left(F^{\times}\right)^{r_{0}}$ consisting of the last $r_{0}$ terms). Then, under the above Hecke algebra isomorphisms, $\operatorname{Ind}_{B}^{G}(\lambda \chi)$ is identified with $\left(\operatorname{Ind}_{B_{1}}^{H_{1}^{\prime}}\left(\lambda_{1}\right)\right) \otimes\left(\operatorname{Ind}_{B_{0}}^{H_{0}^{\prime}}\left(\lambda_{0}\right)\right)$.

Remark 1.1. Because of the technical difficulties involved in dealing with disconnected groups, we will not pursue $O(2 n)$ in detail in this paper. However, in order that we may at least indicate what is expected in that case, 
we briefly discuss how to extend Roche's isomorphisms to get the analogue for $O(2 n)$ of the example above.

Let us continue to assume $F$ of odd residual characteristic. Let $G=$ $O(2 n), \bar{G}=S O(2 n)$, and $\underbrace{\mu \times \cdots \times \mu}_{r_{1}} \times \underbrace{1 \times \cdots \times 1}_{r_{0}}$, where $r_{0}+r_{1}=n$. Let $\tilde{H}=O\left(2 r_{1}\right) \times O\left(2 r_{0}\right)$ and $\tilde{\bar{H}}=S\left(O\left(2 r_{1}\right) \times O\left(2 r_{0}\right)\right)$. By Roche's results, there is a support-preserving isomorphism of Hecke algebras

$$
\bar{\Psi}: \mathcal{H}(\bar{G}, \rho) \longrightarrow \mathcal{H}(\tilde{\bar{H}}, 1) .
$$

We will use $\bar{\Psi}$ to construct a support-preserving isomorphism of Hecke algebras

$$
\Psi: \mathcal{H}(G, \rho) \longrightarrow \mathcal{H}(\tilde{H}, 1) .
$$

To this end, let $i_{G}: \mathcal{H}(\bar{G}, \rho) \longrightarrow \mathcal{H}(G, \rho)$ and $i_{H}: \mathcal{H}(\tilde{H}) \longrightarrow \mathcal{H}(\tilde{H}, 1)$ denote the obvious embeddings. If we let $c_{n}$ denote the $n$th sign change (an element of $O(2 n) \backslash S O(2 n))$ and $C=\left\langle 1, c_{n}\right\rangle$, then we have $\mathcal{H}(G, \rho) \cong \mathcal{H}(\bar{G}, \rho) \tilde{\otimes} \mathbb{C}[C]$. Similarly, if we let $H=S O\left(2 r_{1}\right) \times S O\left(2 r_{0}\right)$ and $C^{\prime}=\left\langle 1, c_{r_{1}}, c_{n}, c_{r_{1}} c_{n}\right\rangle$, $C^{\prime \prime}=\left\langle 1, c_{r_{1}} c_{n}\right\rangle$, then $\mathcal{H}(\tilde{H}, 1) \cong \mathcal{H}(H, 1) \tilde{\otimes} \mathbb{C}\left[C^{\prime}\right], \mathcal{H}(\tilde{\bar{H}}, 1) \cong \mathcal{H}(H, 1) \tilde{\otimes} \mathbb{C}\left[C^{\prime \prime}\right]$. We may then define $\Psi$ by

$$
\begin{aligned}
\Psi: h \otimes 1 & \longmapsto i_{H} \circ \bar{\Psi} \circ i_{G}^{-1}(h) \otimes 1 \\
h \otimes e_{c_{n}} & \longmapsto i_{H} \circ \bar{\Psi} \circ i_{G}^{-1}(h) \otimes e_{c_{n}}^{\prime} .
\end{aligned}
$$

The definition of $\Psi$ ensures that it is a support-preserving linear isomorphism. We need to check that it also respects multiplication. For this, it is enough to show that $c_{n}\left(i_{H} \circ \bar{\Psi} \circ i_{G}^{-1}\left(T_{w}\right)\right)=i_{H} \circ \bar{\Psi} \circ i_{G}^{-1}\left(T_{c_{n}(w)}\right)$. Since $\bar{\Psi}$ is a support-preserving isomorphism, we may write $\bar{\Psi}\left(T_{w}\right)=a_{w} T_{w}^{\prime}$. Then,

$$
c_{n}\left(i_{H} \circ \bar{\Psi} \circ i_{G}^{-1}\left(T_{w}\right)\right)=a_{w} T_{c_{n}(w)}^{\prime}
$$

and

$$
i_{H} \circ \bar{\Psi} \circ i_{G}^{-1}\left(T_{c_{n}(w)}\right)=a_{c_{n}(w)} T_{c_{n}(w)}^{\prime} .
$$

Therefore, it is enough to show that $a_{w}=a_{c_{n}(w)}$. For this, it is enough to show that $a_{w}=a_{c_{n}(w)}$ for $w \in \bar{W}_{\chi}=\left\{w \in \bar{W}_{\bar{G}} \mid w \cdot \chi=\chi\right\}$ and $w=y \in$ $Y=\operatorname{Hom}\left(G_{m}, T\right)$. Now, since $c_{n} \cdot \Pi=\Pi$ and $c_{n} \cdot \Pi_{\chi}=\Pi_{\chi}$ (cf. $[\mathbf{R}$, p. 393]), we have $\ell\left(c_{n}(w)\right)=\ell(w)$ and $\ell_{\chi}\left(c_{n}(w)\right)=\ell_{\chi}(w)$ for $w \in \bar{W}_{\chi}$, where $\ell_{\chi}=\ell_{H}$ denotes length taken with respect to $\Pi_{\chi}$. Since $\bar{\Psi}: q^{-\frac{1}{2} \ell(w)} T_{w} \longmapsto$ $q^{-\frac{1}{2} \ell_{\chi}(w)} T_{w}^{\prime}$, this tells us that $a_{w}=a_{c_{n}(w)}$ for $w \in \bar{W}_{\chi}$. This also tells us that $\delta \circ c_{n}=\delta$ and $\delta_{H} \circ c_{n}=\delta_{H}$. Therefore, $q^{\ell(y)}=q^{\ell\left(c_{n}(y)\right)}$ for $y \in Y^{+}=$ $\left\{y \in Y \mid\langle y, \alpha\rangle \geq 0\right.$ for all $\left.\alpha \in \Phi^{+}\right\}$(cf. [Ca, Lemma 1.5.1]), and similarly for $\ell_{\chi}=\ell_{H}$. Since $\bar{\Psi}: \delta^{\frac{1}{2}}(y) T_{y} \longmapsto \delta_{H}^{\frac{1}{2}}(y) T_{y}^{\prime}$ (cf. proof of Lemma $9.3[\mathbf{R}]$ ), it follows that $a_{y}=a_{c_{n}(y)}$ for $y \in Y^{+}$, as needed. 
The following results describe what happens when $\chi$ is replaced by $\chi^{-1}$. They will be needed below.

Lemma 1.1. In Roche's construction, if $(J, \rho)$ is the type associated to $\chi$, then the type associated to $\chi^{-1}$ is $\left(J, \rho^{-1}\right)$.

Proof. Write $\left(J_{\chi}, \rho_{\chi}\right),\left(J_{\chi^{-1}}, \rho_{\chi^{-1}}\right)$ for the types associated to $\chi, \chi^{-1}$, respectively. The functions $f_{\chi}$ and $f_{\chi^{-1}}$ from Definition $3.3[\mathbf{R}]$ must be the same. Therefore, $J_{\chi}=J_{f_{\chi}}$ and $J_{\chi^{-1}}=J_{f_{\chi^{-1}}}$ must be the same. It is then immediate from the definitions that $\rho_{\chi^{-1}}=\left(\rho_{\chi}\right)^{-1}$ (cf. $[\mathbf{R}$, Proposition $3.6])$.

Corollary 1.2. $\mathcal{H}(G, \rho) \cong \mathcal{H}\left(G, \rho^{-1}\right)$ via a support-preserving isomorphism. (The isomorphism may also be taken to be *-preserving; cf. Section $6[\mathbf{R}]$.)

Proof. Observe that in the notation of $[\mathbf{R}]$, we have $\Phi_{\chi}=\Phi_{\chi^{-1}}, \Phi_{\chi, a f}=$ $\Phi_{\chi^{-1}, a f}$ (cf. [R, Definition 6.1]) and $W_{\chi}=W_{\chi^{-1}}$ (cf. [R, Theorem 4.14]). Thus, $\mathcal{C}_{\chi}=\mathcal{C}_{\chi^{-1}}$, and therefore $\Pi_{\chi, a f}=\Pi_{\chi^{-1}, a f}$ (cf. [R, Section 6]). It then follows that $S_{\chi}^{0}=S_{\chi^{-1}}^{0}$ and $\Omega_{\chi}=\Omega_{\chi^{-1}}$ (also [R, Section 6]). The corollary is now an immediate consequence of $[\mathbf{R}$, Theorem 6.3].

We now define an involution $j$ on $\mathcal{H}\left(H, 1_{I}\right) \tilde{\otimes} \mathbb{C}\left[C_{\chi}\right]$. For $h \in \mathcal{H}\left(H, 1_{I}\right)$, $j(h)$ is defined as above. For $\mathbb{C}\left[C_{\chi}\right]$, fix a character $\sigma: C_{\chi} \longrightarrow \mathbb{C}^{\times}$with $\sigma^{2}=1$. Then, for $c \in C_{\chi}$, set $j\left(e_{c}\right)=\sigma(c) e_{c}$. This extends to an involution of $\mathbb{C}\left[C_{\chi}\right]$ (trivial if $\sigma=1$ ). We extend $j$ to $\mathcal{H}\left(H, 1_{I}\right) \tilde{\otimes} \mathbb{C}\left[C_{\chi}\right]$ bilinearly.

Proposition 1.3. $j$ is an involution of $\mathcal{H}\left(H, 1_{I}\right) \tilde{\otimes} \mathbb{C}\left[C_{\chi}\right]$.

Proof. We need to check that $j$ respects multiplication. For $h_{1}, h_{2} \in \mathcal{H}\left(H, 1_{I}\right)$ and $c_{1}, c_{2} \in C_{\chi}$, we have

$$
j\left(h_{1} \otimes e_{c_{1}}\right) \cdot j\left(h_{2} \otimes e_{c_{2}}\right)=\sigma\left(c_{1} c_{2}\right)\left[j\left(h_{1}\right) c_{1}\left(j\left(h_{2}\right)\right) \otimes e_{c_{1} c_{2}}\right]
$$

and

$$
j\left(\left(h_{1} \otimes e_{c_{1}}\right) \cdot\left(h_{2} \otimes e_{c_{2}}\right)\right)=\sigma\left(c_{1} c_{2}\right)\left[j\left(h_{1}\right) j\left(c_{1}\left(h_{2}\right)\right) \otimes e_{c_{1} c_{2}}\right] .
$$

Thus, it suffices to show $j(c(h))=c(j(h))$ for $h \in \mathcal{H}\left(H, 1_{I}\right), c \in C_{\chi}$. Since the action of $C_{\chi}$ on $H$ preserves the set of simple roots of $H$, hence $\delta_{H}$ and $\ell_{H}$, we see that

$$
j\left(c\left(T_{w}\right)\right)=(-q)^{\ell_{H}(w)}\left(T_{c\left(w^{-1}\right)}\right)^{-1}=c\left(j\left(T_{w}\right)\right)
$$

for all $w \in \bar{W}_{H}$. Also, since $c\left(\theta_{t}\right)=\theta_{c(t)}$, we get

$$
j\left(c\left(\theta_{t}\right)\right)=\theta_{c(t)}^{-1}=c\left(j\left(\theta_{t}\right)\right),
$$

as needed. 
We now consider how these restrict to Levi subgroups of standard parabolics. First, if $M$ is a standard Levi of $\mathrm{G}$, we have support-preserving isomorphisms

$$
\begin{gathered}
\Psi_{G}: \mathcal{H}(G, \rho) \longrightarrow \mathcal{H}\left(H, 1_{I}\right) \tilde{\otimes} \mathbb{C}\left[C_{\chi}\right]=\mathcal{H}\left(\tilde{H}, 1_{I_{H}}\right) \\
\Psi_{M}: \mathcal{H}\left(M, \rho_{M}\right) \longrightarrow \mathcal{H}\left(L, 1_{I_{L}}\right) \tilde{\otimes} \mathbb{C}\left[D_{\chi}\right]=\mathcal{H}\left(\tilde{L}, 1_{I_{L}}\right)
\end{gathered}
$$

constructed by Roche. In general, these are not unique. Similarly, let $j_{H}$, resp. $j_{L}$, denote the Iwahori-Matsumoto involutions for $\mathcal{H}\left(H, 1_{I}\right) \tilde{\otimes} \mathbb{C}\left[C_{\chi}\right]$, resp. $\mathcal{H}\left(L, 1_{I}\right) \tilde{\otimes} \mathbb{C}\left[D_{\chi}\right]$ constructed above. We note the following:

Lemma 1.4. We have $D_{\chi} \subset C_{\chi}$ and may take $L$ to be a standard Levi subgroup of $H$.

Proof. First, we check that $D_{\chi} \subset C_{\chi}$. In fact, we claim that $D_{\chi}=C_{\chi} \cap \bar{W}_{M}$. Recall that

$$
D_{\chi}=\left\{w \in \bar{W}_{M, \chi} \mid w \cdot \Phi_{M, \chi}^{+}=\Phi_{M, \chi}^{+}\right\} .
$$

Now, it is immediate from the definitions that $\bar{W}_{M, \chi}=\bar{W}_{M} \cap \bar{W}_{\chi}$ and $\Phi_{M, \chi}^{+}=\Phi_{M} \cap \Phi_{\chi}^{+}$. Therefore, if $w \in C_{\chi} \cap \bar{W}_{M}$, we have $w \in \bar{W}_{M, \chi}$. Further, $w \cdot \Phi_{\chi}^{+}=\Phi_{\chi}^{+}\left(\right.$since $\left.w \in C_{\chi}\right)$ and $w \cdot \Phi_{M}=\Phi_{M}\left(\right.$ since $\left.w \in \bar{W}_{M}\right)$, implying $w \cdot \Phi_{M, \chi}^{+}=\Phi_{M, \chi}^{+}$. Thus, $C_{\chi} \cap \bar{W}_{M} \subset D_{\chi}$. On the other hand, suppose $d \in D_{\chi}$. It is automatic that $d \in \bar{W}_{M}$. To show $d \in C_{\chi} \cap \bar{W}_{M}$, it remains to check that $d \cdot \Phi_{\chi}^{+}=\Phi_{\chi}^{+}$. From the definition of $D_{\chi}$, we have $d \cdot \Phi_{M, \chi}^{+}=\Phi_{M, \chi}^{+}$. Suppose $\alpha \in \Phi_{\chi}^{+} \backslash \Phi_{M, \chi}^{+}$. Since $\Phi_{M, \chi}^{+}=\Phi_{M} \cap \Phi_{\chi}^{+}$, this forces $\alpha \in \Phi^{+} \backslash \Phi_{M}^{+}$. As $\bar{W}_{M} \cdot\left(\Phi^{+} \backslash \Phi_{M}^{+}\right)=\Phi^{+} \backslash \Phi_{M}^{+}$, we have $d \cdot \alpha \in \Phi^{+} \backslash \Phi_{M}^{+}$. Thus, $d \cdot \alpha \in\left(\Phi^{+} \backslash \Phi_{M}^{+}\right) \cap \Phi_{\chi} \subset \Phi_{\chi}^{+}$, as needed. This tells us $D_{\chi} \subset C_{\chi} \cap \bar{W}_{M}$. Thus, $D_{\chi}=C_{\chi} \cap \bar{W}_{M}$, as claimed.

Now, we identify $L$ as a standard Levi subgroup of $H$. First, we claim that if $\Pi_{M, \chi}$ denotes the simple roots in $\Phi_{M, \chi}$, then $\Pi_{M, \chi}=\Pi_{\chi} \cap \Phi_{M, \chi}$. Certainly, if $\alpha \in \Pi_{\chi} \cap \Phi_{M, \chi}$, then $\alpha \in \Pi_{M, \chi}$ (minimal with respect to $\Phi_{\chi}^{+}$implies minimal with respect to $\Phi_{M, \chi}^{+}=\Phi_{M} \cap \Phi_{\chi}^{+}$). In the other direction, suppose $\alpha \in \Pi_{M, \chi}$ but $\alpha \notin \Pi_{\chi} \cap \Phi_{M, \chi}$. Since $\Pi_{M, \chi} \subset \Phi_{M, \chi}$, this requires $\alpha \notin \Pi_{\chi}$. Therefore, we can write $\alpha=\beta+\gamma$ with $\beta, \gamma \in \Phi_{\chi}^{+}$. Write $\alpha=\sum a_{i} \alpha_{i}$, $\beta=\sum b_{i} \alpha_{i}, \gamma=\sum c_{i} \alpha_{i}$, where $\alpha_{i}$ runs over the simple roots $\Pi$ of $G$. Then, $a_{i}=b_{i}+c_{i}$ for all $i$. Further, $a_{i}, b_{i}, c_{i} \geq 0$ (since $\left.\alpha, \beta, \gamma \in \Phi_{\chi}^{+} \subset \Phi^{+}\right)$. Now, $\alpha \in \Phi_{M}$ implies $a_{i}=0$ for $\alpha_{i} \notin \Pi_{M}$. Therefore, $b_{i}=c_{i}=0$ for $\alpha_{i} \notin \Pi_{M}$. Thus, $\beta, \gamma \in \Phi_{M}$. In particular, $\beta, \gamma \in \Phi_{\chi}^{+} \cap \Phi_{M}=\Phi_{M, \chi}^{+}$. However, the decomposition $\alpha=\beta+\gamma$ with $\beta, \gamma \in \Phi_{M, \chi}^{+}$contradicts the simplicity of $\alpha$ in $\Phi_{M, \chi}^{+}$. Thus, $\Pi_{M, \chi}=\Pi_{\chi} \cap \Phi_{M, \chi}$, as claimed. We now let $L$ be the Levi factor of the standard parabolic subgroup of $H$ associated to the subset of simple roots $\Pi_{M, \chi} \subset \Pi_{\chi}=\Pi_{H}$. Then, $L$ has the right root data to appear in the isomorphism $\mathcal{H}\left(M, \rho_{M}\right) \cong \mathcal{H}\left(L, 1_{I_{L}}\right) \tilde{\otimes} \mathbb{C}\left[D_{\chi}\right]$. 
The next step is to identify $\mathcal{H}\left(M, \rho_{M}\right)$ as a subalgebra of $\mathcal{H}(G, \rho)$. Let $P=M U$ be the standard parabolic subgroup with Levi factor $M$; $\bar{U}$ the unipotent radical opposite $U$. Set

$$
J_{\ell}=J \cap \bar{U}, \quad J_{M}=J \cap M, \quad J_{u}=J \cap U,
$$

which gives $J=J_{\ell} J_{M} J_{u}$. Let

$$
\mathcal{I}_{M}^{+}=\left\{w \in W_{M} \mid w J_{u} w^{-1} \subset J_{u}, w^{-1} J_{\ell} w \subset J_{\ell}\right\} .
$$

We set

$$
\mathcal{I}_{M, \chi}^{+}=\mathcal{I}_{M}^{+} \cap W_{M, \chi}
$$

If we let $\mathcal{H}^{+}\left(M, \rho_{M}\right)$ denote the space of functions in $\mathcal{H}\left(M, \rho_{M}\right)$ with support contained in $J_{M} \mathcal{I}_{M, \chi}^{+} J_{M}$, then there is a support-preserving embedding (of $\mathbb{C}$-algebras with 1 )

$$
\mathcal{H}^{+}\left(M, \rho_{M}\right) \hookrightarrow \mathcal{H}(G, \rho)
$$

([Bu-K, Corollary 6.12]). This may be extended uniquely to an embedding

$$
\mathcal{H}\left(M, \rho_{M}\right) \hookrightarrow \mathcal{H}(G, \rho)
$$

([Bu-K, Theorem 7.2], noting that the existence of $\zeta$ as in the hypotheses of Theorem $7.2[\mathbf{B u}-\mathbf{K}]$ follows immediately from the support-preserving isomorphism of Roche and the fact that elements of $\mathcal{H}\left(\tilde{H}, 1_{I_{H}}\right)$ supported on a single double-coset are invertible). We fix such embeddings $\mathcal{H}\left(M, \rho_{M}\right) \hookrightarrow$ $\mathcal{H}(G, \rho)$ and $\mathcal{H}\left(\tilde{L}, 1_{I_{L}}\right) \hookrightarrow \mathcal{H}\left(\tilde{H}, 1_{I_{H}}\right)$.

Now, we note that $W_{M, \chi}=\bar{W}_{M, \chi} \cdot Y$, where $Y=\operatorname{Hom}\left(G_{m}, T\right)$ (which may be viewed as a set of representatives for $T / T(\mathcal{O})$ ). If we let

$$
Y_{M, \chi}^{+}=\mathcal{I}_{M, \chi}^{+} \cap Y,
$$

we have the following:

\section{Lemma 1.5 .}

$$
\mathcal{I}_{M, \chi}^{+}=\bar{W}_{M, \chi} \cdot Y_{M, \chi}^{+}
$$

Proof. First, we check that $\bar{W}_{M, \chi} \cdot Y_{M, \chi}^{+} \subset \mathcal{I}_{M, \chi}^{+}$. By definition, $Y_{M, \chi}^{+} \subset \mathcal{I}_{M, \chi}^{+}$. Thus, we need only check that $\bar{W}_{M, \chi} \subset \mathcal{I}_{M, \chi}^{+}$. Recall that Roche defines

$$
f_{\chi}(\alpha)=\left\{\begin{array}{ll}
{\left[c_{\alpha} / 2\right],} & \text { for } \alpha \in \Phi^{+}, \\
{\left[\left(c_{\alpha}+1\right) / 2\right],} & \text { for } \alpha \in \Phi^{-},
\end{array},\right.
$$

where $c_{\alpha}=\operatorname{cond}(\chi \circ \check{\alpha})$ (where $\operatorname{cond}(\lambda)$ is defined to be the lowest positive integer such that $\left.1+\mathfrak{p}^{n} \subset \operatorname{ker}(\lambda)\right)$. Then,

$$
J_{\ell}=\prod_{\alpha \in \Phi^{-} \backslash \Phi_{M}^{-}} U_{\alpha, f_{\chi}(\alpha)}, \quad J_{u}=\prod_{\alpha \in \Phi^{+} \backslash \Phi_{M}^{+}} U_{\alpha, f_{\chi}(\alpha)},
$$




$$
J_{M}=\left(\prod_{\alpha \in \Phi_{M}^{-}} U_{\alpha, f_{\chi}(\alpha)}\right) \cdot A(\mathcal{O}) \cdot\left(\prod_{\alpha \in \Phi_{M}^{+}} U_{\alpha, f_{\chi}(\alpha)}\right)
$$

Here, if $\alpha \in \Phi^{+}$,

$$
U_{\alpha, k}=\phi_{\alpha}\left(\begin{array}{cc}
1 & \mathfrak{p}^{k} \\
0 & 1
\end{array}\right), \quad U_{-\alpha, k}=\phi_{\alpha}\left(\begin{array}{cc}
1 & 0 \\
\mathfrak{p}^{k} & 1
\end{array}\right),
$$

with $\phi_{\alpha}: S L_{2} \longrightarrow G$ as usual. Now, suppose $w \in \bar{W}_{M}$ and $\alpha \in \Phi^{+}$. Then,

$$
w \cdot U_{\alpha, f_{\chi}(\alpha)}=\phi_{w \cdot \alpha}\left(\begin{array}{cc}
1 & \mathfrak{p}^{f_{\chi}(\alpha)} \\
0 & 1
\end{array}\right)
$$

suitably interpreted if $w \cdot \alpha<0$. To show that $w J_{u} w^{-1} \subset J_{u}$ for $w \in \bar{W}_{M, \chi}$, it is (more than) enough to show that if $\alpha \in \Phi^{+} \backslash \Phi_{M}^{+}$, then (1) $w \cdot \alpha \in$ $\Phi^{+}$and $(2) f_{\chi}(w \cdot \alpha)=f_{\chi}(\alpha)$. Of course, (1) follows immediately from $w \cdot\left(\Phi^{+} \backslash \Phi_{M}^{+}\right)=\Phi^{+} \backslash \Phi_{M}^{+}$(which holds for any $w \in \bar{W}_{M}$ ). (2) follows from $w \cdot \chi=\chi:\left(w^{-1} \cdot \chi\right) \circ \check{\alpha}=\chi \circ \check{\alpha}$ implies $c_{w \cdot \alpha}=c_{\alpha}$, so that $f_{\chi}(w \cdot \alpha)=f_{\chi}(\alpha)$, as needed. The same argument may be used to show $w^{-1} J_{\ell} w \subset J_{\ell}$. Thus, we get $\bar{W}_{M, \chi} \subset \mathcal{I}_{M, \chi}^{+}$, as needed. In fact, this shows more: for $w \in \bar{W}_{M, \chi}$, we have $w J_{u} w^{-1}=J_{u}$ and $w^{-1} J_{\ell} w=J_{\ell}$.

The containment $\mathcal{I}_{M, \chi}^{+} \subset \bar{W}_{M, \chi} \cdot Y_{M, \chi}^{+}$is now easy. Take

$$
x \in \mathcal{I}_{M, \chi}^{+} \subset W_{M, \chi}=\bar{W}_{M, \chi} \cdot Y
$$

and write $x=w y, w \in \bar{W}_{M, \chi}, y \in Y$. Then, it suffices to show that $y \in Y_{M, \chi}^{+}$. Calculate:

$$
(w y) J_{u}(w y)^{-1} \subset J_{u} \Longrightarrow y J_{u} y^{-1} \subset w^{-1} J_{u} w=J_{u}
$$

since $w \in \bar{W}_{M, \chi} \subset \mathcal{I}_{M, \chi}^{+}$. Similarly, $y^{-1} J_{\ell} y \subset J_{\ell}$. Thus, $y \in Y_{M, \chi}^{+}$, as needed.

Now, we can prove the following:

Proposition 1.6. (1) We may take $\Psi_{M}=\left.\Psi_{G}\right|_{\mathcal{H}\left(M, \rho_{M}\right)}$ to get a supportpreserving isomorphism

$$
\Psi_{M}: \mathcal{H}\left(M, \rho_{M}\right) \longrightarrow \mathcal{H}\left(L, 1_{I_{L}}\right) \tilde{\otimes} \mathbb{C}\left[D_{\chi}\right] .
$$

(2) If we let $j_{H}$, resp. $j_{L}$, denote the Iwahori-Matsumoto involutions for $\mathcal{H}\left(H, 1_{I}\right) \tilde{\otimes} \mathbb{C}\left[C_{\chi}\right]$, resp. $\mathcal{H}\left(L, 1_{I_{L}}\right) \tilde{\otimes} \mathbb{C}\left[D_{\chi}\right]$, then

$$
j_{L}=\left.j_{H}\right|_{\mathcal{H}\left(L, 1_{I_{L}}\right) \tilde{\otimes} \mathbb{C}\left[D_{\chi}\right]} .
$$

Proof. We first say a few words about what it means for $\Psi_{G}$ to be supportpreserving. By construction, $Y$ is associated to both $G$ and $H$; we use $Y_{G}, Y_{H}$ when we wish to distinguish the context. Note that $W_{\chi}=Y \rtimes \bar{W}_{\chi}$. Also, since $\Phi_{H}=\Phi_{\chi}$, we can identify $\bar{W}_{H}^{0}$ and $\bar{W}_{\chi}^{0}$. This gives rise to 
an identification of $\bar{W}_{\chi}=\bar{W}_{\chi}^{0} \rtimes C_{\chi}$ with $\bar{W}_{H}^{0} \rtimes C_{\chi}$. Thus, we have an identification

$$
\psi_{G}: W_{\chi}=Y \rtimes \bar{W}_{\chi} \longleftrightarrow Y \rtimes\left(\bar{W}_{H}^{0} \rtimes C_{\chi}\right)=W_{H} \rtimes C_{\chi} .
$$

Then, $\Psi_{G}: \mathcal{H}(G, \rho) \longrightarrow \mathcal{H}\left(H, 1_{I}\right) \tilde{\otimes} \mathbb{C}\left[C_{\chi}\right]$ is support-preserving means that if $T \in \mathcal{H}(G, \rho)$ is supported on $J w J$, then $\Psi_{G}(T)$ is supported on $I_{H} \psi_{G}(w) I_{H}$.

Now, to make matters precise, let $t_{M}: \mathcal{H}\left(M, \rho_{M}\right) \hookrightarrow \mathcal{H}(G, \rho)$ and $t_{\tilde{L}}$ : $\mathcal{H}\left(\tilde{L}, 1_{I_{L}}\right) \hookrightarrow \mathcal{H}\left(\tilde{H}, 1_{I_{H}}\right)$ denote the fixed embeddings. To verify (1), we need to show that

$$
\Psi_{G}\left(t_{M}\left(\mathcal{H}\left(M, \rho_{M}\right)\right)\right)=t_{\tilde{L}}\left(\mathcal{H}\left(\tilde{L}, 1_{I_{L}}\right)\right) .
$$

To this end, we first show that

$$
\Psi_{G}\left(t_{M}\left(\mathcal{H}^{+}\left(M, \rho_{M}\right)\right)\right) \subset t_{\tilde{L}}\left(\mathcal{H}^{+}\left(\tilde{L}, 1_{I_{L}}\right)\right) .
$$

Since the restriction of $t_{M}$ to $\mathcal{H}^{+}\left(M, \rho_{M}\right)$ is support-preserving, and similarly for $t_{\tilde{L}}$, it is enough to show that

$$
\psi_{G}\left(\mathcal{I}_{M, \chi}^{+}\right) \subset \mathcal{I}_{\tilde{L}}^{+}=\bar{W}_{L} \cdot D_{\chi} \cdot Y_{L}^{+} .
$$

By Lemma 1.5 and the fact that $\psi_{G}\left(\bar{W}_{M, \chi}\right)=\bar{W}_{L} \cdot D_{\chi}$, we are reduced to checking that $\psi_{G}\left(Y_{M, \chi}^{+}\right) \subset Y_{L}^{+}$. Now, if $y \in Y$ and $\alpha \in \Phi$, then $y U_{\alpha, k} y^{-1}=$ $U_{\alpha, k+\alpha(y)}$. Therefore, $y \in Y_{M, \chi}^{+}$if and only if $\alpha(y) \geq 0$ for all $\alpha \in \Phi^{+} \backslash \Phi_{M}^{+}$ (noting that the corresponding condition for negative roots also reduces to this). On the other hand, $y \in Y_{L}^{+}$if and only if $\alpha(y) \geq 0$ for all $\alpha \in$ $\Phi_{H}^{+} \backslash \Phi_{L}^{+}=\Phi_{\chi}^{+} \backslash \Phi_{M, \chi}^{+} \subset \Phi^{+} \backslash \Phi_{M}^{+}$, so we see that $\psi_{G}\left(Y_{M, \chi}^{+}\right) \subset Y_{L}^{+}$, as needed.

We now proceed to show that

$$
\Psi_{G}\left(t_{M}\left(\mathcal{H}\left(M, \rho_{M}\right)\right)\right)=t_{\tilde{L}}\left(\mathcal{H}\left(\tilde{L}, 1_{I_{L}}\right)\right) .
$$

First, choose $\zeta$ and $\phi_{m}$ as in Proposition $7.1[\mathbf{B u}-\mathbf{K}]$. Let $y_{0} \in Y_{M, \chi}^{+}$ be a representative for $\zeta$. Then, $\left(\phi_{m}\right)^{-1}=\phi_{-m} \in \mathcal{H}\left(M, \rho_{M}\right)$. We note that $\mathcal{H}^{+}\left(M, \rho_{M}\right)$ and $\phi_{-1}$ are sufficient to generate $\mathcal{H}\left(M, \rho_{M}\right)$. We already have $\Psi_{G}\left(t_{M}\left(\mathcal{H}^{+}\left(M, \rho_{M}\right)\right)\right) \subset t_{\tilde{L}}\left(\mathcal{H}^{+}\left(\tilde{L}, 1_{I_{L}}\right)\right)$. Since $y_{0} \in \mathcal{I}_{M, \chi}^{+}$, we have $\Psi_{G}\left(t_{M}\left(\phi_{1}\right)\right) \in t_{\tilde{L}}\left(\mathcal{H}^{+}\left(\tilde{L}, 1_{I_{L}}\right)\right)$. Further, since $\left.t_{M}\right|_{\mathcal{H}^{+}\left(M, \rho_{M}\right)}$ and $\Psi_{G}$ are support-preserving, $\Psi_{G}\left(t_{M}\left(\phi_{1}\right)\right)$ is supported on the double-coset $I_{H} y_{0} I_{H}$. Therefore, $\Psi_{G}\left(t_{M}\left(\phi_{1}\right)\right)=t_{\tilde{L}}\left(\phi_{1}^{\prime}\right)$, where $\phi_{1}^{\prime} \in \mathcal{H}\left(\tilde{L}, 1_{I_{L}}\right)$ is supported on the double-coset $I_{L} y_{0} I_{L}$. As a consequence, $\phi_{1}^{\prime}$ is invertible and $\Psi_{G}\left(t_{M}\left(\phi_{1}^{-1}\right)\right)=$ $t_{\tilde{L}}\left(\phi_{1}^{\prime-1}\right)$. Since $\mathcal{H}^{+}\left(M, \rho_{M}\right)$ and $\phi_{1}^{-1}$ are enough to generate $\mathcal{H}\left(M, \rho_{M}\right)$, we have $\Psi_{G}\left(t_{M}\left(\mathcal{H}\left(M, \rho_{M}\right)\right)\right) \subset t_{\tilde{L}}\left(\mathcal{H}\left(\tilde{L}, 1_{I_{L}}\right)\right)$. On the other hand, we know that $\Psi_{G}\left(t_{M}\left(\mathcal{H}\left(M, \rho_{M}\right)\right)\right)$ contains the functions $\left\{t_{\tilde{L}}\left(T_{w}\right) \mid w \in \psi_{G}\left(\bar{W}_{M, \chi}\right)=\right.$ $\left.\bar{W}_{L} D_{\chi}\right\},\left\{t_{\tilde{L}}\left(T_{y}\right) \mid y \in Y_{M, \chi}^{+}\right\}$, and $t_{\tilde{L}}\left(T_{y_{0}}^{-1}\right)$. As these are enough to generate $t_{\tilde{L}}\left(\mathcal{H}\left(\tilde{L}, 1_{I_{L}}\right)\right)$, we see that

$$
\Psi_{G}\left(t_{M}\left(\mathcal{H}\left(M, \rho_{M}\right)\right)\right)=t_{\tilde{L}}\left(\mathcal{H}\left(\tilde{L}, 1_{I_{L}}\right)\right),
$$


as needed.

We now know that $t_{\tilde{L}}^{-1} \circ \Psi_{G} \circ t_{M}$ is an isomorphism from $\mathcal{H}\left(M, \rho_{M}\right)$ to $\mathcal{H}\left(\tilde{L}, 1_{I_{L}}\right)$. We must show that it is support-preserving. To this end, observe that since $\zeta \in Z(M)$, we have $\alpha\left(y_{0}\right)=0$ for all $\alpha \in \Phi_{M}$. Therefore, viewing $y_{0}$ as an element of $Y_{H}$, we have $\alpha\left(y_{0}\right)=0$ for all $\alpha \in \Phi_{L}=\Phi_{M, \chi}$. In particular, $y_{0} U_{\alpha, k} y_{0}^{-1}=U_{\alpha, k+\alpha\left(y_{0}\right)}=U_{\alpha, k}$ for all $\alpha \in \Phi_{L}$ allows us to conclude that $I_{L} y_{0}=y_{0} I_{L}$. Therefore, in $\mathcal{H}\left(\tilde{L}, 1_{I_{L}}\right)$, we have that $T_{y_{0}} T_{w}$ is supported on $I_{L} y_{0} w I_{L}$ for any $w \in W_{L} D_{\chi}$. In particular, $T_{y_{0}}^{-1}$ is supported on $I_{L} y_{0}^{-1} I_{L}$. Thus, we have that $t_{\tilde{L}}^{-1} \circ \Psi_{G} \circ t_{M}$ is support-preserving on $\mathcal{H}^{+}\left(M, \rho_{M}\right)$ and $\phi_{1}^{-1}=\phi_{-1}$. By writing $x \in W_{M, \chi}$ as $y_{0}^{-m} w$, with $w \in$ $W_{M, \chi}^{+}\left([\mathbf{B u}-\mathbf{K}\right.$, Lemma 6.14] $)$, one can then check that $t_{\tilde{L}}^{-1} \circ \Psi_{G} \circ t_{M}\left(T_{x}\right)$ is supported on $I_{L} y_{0}^{-m} \psi_{G}(w) I_{L}$, as needed.

It is now straightforward to check the second claim. This follows immediately from the following observations:

(1) Since $L$ is a standard Levi of $H$, the Iwahori-Matsumoto involution on $\mathcal{H}\left(H, 1_{I}\right)$ restricts to give the Iwahori-Matsumoto involution on $\mathcal{H}\left(L, 1_{I_{L}}\right)$.

(2) Since $D_{\chi} \subset C_{\chi}$, we may choose $\left.\sigma\right|_{D_{\chi}}$ for the character of order $\leq 2$ of $D_{\chi}$.

This finishes the proof of the proposition.

Suppose $\mathcal{H}_{1}, \mathcal{H}_{2}$ are Hecke algebras and $\phi: \mathcal{H}_{1} \longrightarrow \mathcal{H}_{2}$ is an isomorphism. Suppose $\pi$ is a representation of $\mathcal{H}_{1}$ with space $V$. Let $\phi(\pi)$ denote the corresponding representation of $\mathcal{H}_{2}$ (i.e., $\mathcal{H}_{2}$ acting on $V$ by $\pi \circ \phi^{-1}$ ). We define induction as in $[\mathbf{B u}-\mathbf{K}]$ : If $\mathcal{L}_{1} \subset \mathcal{H}_{1}$ is a subalgebra and $\tau$ is a representation of $\mathcal{L}_{1}$, then $\operatorname{Ind}_{\mathcal{L}_{1}}^{\mathcal{H}_{1}} \tau$ has space $\operatorname{Hom}_{\mathcal{L}_{1}}\left(\mathcal{H}_{1}, V_{\tau}\right)$ (where $\mathcal{H}_{1}$ is an $\mathcal{L}_{1}$-module by left multiplication). The action is right translation. We claim that $\phi$ respects induction. In particular, if $\mathcal{L}_{2}=\phi\left(\mathcal{L}_{1}\right)$, we have the following:

\section{Lemma 1.7.}

$$
\phi\left(\operatorname{Ind}_{\mathcal{L}_{1}}^{\mathcal{H}_{1}} \tau\right) \cong \operatorname{Ind}_{\mathcal{L}_{2}}^{\mathcal{H}_{2}} \phi(\tau) .
$$

Proof. Let $X \in \operatorname{Hom}_{\mathcal{L}_{1}}\left(\mathcal{H}_{1}, V_{\tau}\right)$. Then, it is straightforward to check that $X \circ \phi^{-1} \in \operatorname{Hom}_{\mathcal{L}_{2}}\left(\mathcal{H}_{2}, V_{\phi(\tau)}\right)$ (noting that as a vector space, $V_{\phi(\tau)}=V_{\tau}$; it is written as $V_{\phi(\tau)}$ to indicate the representation). In particular, $\mathcal{E}: X \longrightarrow$ $X \circ \phi^{-1}$ is a bijective linear map from $\operatorname{Hom}_{\mathcal{L}_{1}}\left(\mathcal{H}_{1}, V_{\tau}\right)$ to $\operatorname{Hom}_{\mathcal{L}_{2}}\left(\mathcal{H}_{2}, V_{\phi(\tau)}\right)$.

We claim that $\mathcal{E}: \operatorname{Hom}_{\mathcal{L}_{1}}\left(\mathcal{H}_{1}, V_{\tau}\right) \longrightarrow \operatorname{Hom}_{\mathcal{L}_{2}}\left(\mathcal{H}_{2}, V_{\phi(\tau)}\right)$ gives the desired equivalence. In particular, if $R_{1}$ (resp. $R_{2}$ ) denotes right translation on $\operatorname{Hom}_{\mathcal{L}_{1}}\left(\mathcal{H}_{1}, V_{\tau}\right)\left(\operatorname{resp} . \operatorname{Hom}_{\mathcal{L}_{2}}\left(\mathcal{H}_{2}, V_{\phi(\tau)}\right)\right)$, this requires

$$
\mathcal{E} R_{1}\left(\phi^{-1}\left(h_{2}\right)\right) X=R_{2}\left(h_{2}\right) \mathcal{E} X
$$


for all $X \in \operatorname{Hom}_{\mathcal{L}_{1}}\left(\mathcal{H}_{1}, V_{\tau}\right), h_{2} \in \mathcal{H}_{2}$. Now, it is easy to check that

$$
\left[\mathcal{E} R_{1}\left(\phi^{-1}\left(h_{2}\right)\right) X\right]\left(h_{2}^{\prime}\right)=X\left(\phi^{-1}\left(h_{2}^{\prime} h_{2}\right)\right)=\left[R_{2}\left(h_{2}\right) \mathcal{E} X\right]\left(h_{2}^{\prime}\right) .
$$

The desired equivalence follows.

We now discuss the implications for the representation theory of $G$. First, if $(\pi, G, V)$ is a representation of $G$, let $\left(\pi, \mathcal{H}(G, \rho), V^{\rho}\right)$ denote the corresponding Hecke algebra representation, where $V^{\rho}=\pi\left(e_{\rho}\right) V$ (the action is inherited from $\mathcal{H}(G)$ acting on $V)$. Now, fix a character $\tilde{\chi}$ extending $\chi$ to $T$. Let $\mathcal{R}_{\chi}(G)$ denote the category of smooth representations having the property that every irreducible subquotient of $\pi$ has supercuspidal support contained in $\{\lambda(w \cdot \tilde{\chi}) \mid w \in W, \lambda$ an unramified character of $T\}$. As mentioned earlier, $(J, \chi)$ is a type. This means, among other things, that the map $(\pi, G, V) \longmapsto\left(\pi, \mathcal{H}(G, \rho), V^{\rho}\right)$ gives an equivalence of categories between $\mathcal{R}_{\chi}(G)$ and the category of $\mathcal{H}(G, \rho)$ modules. This extends the results of Borel [Bo] and Casselman [Ca] on unramified principal series.

We now discuss the Iwahori-Matsumoto involution on representations. Let

$$
\begin{gathered}
\Psi_{G, \chi}: \mathcal{H}(G, \rho) \longrightarrow \mathcal{H}\left(H, 1_{I}\right) \tilde{\otimes} \mathbb{C}\left[C_{\chi}\right] \\
\Psi_{G, \chi^{-1}}: \mathcal{H}\left(G, \rho^{-1}\right) \longrightarrow \mathcal{H}\left(H, 1_{I}\right) \tilde{\otimes} \mathbb{C}\left[C_{\chi^{-1}}\right]
\end{gathered}
$$

denote the isomorphisms above. Since $C_{\chi}=C_{\chi^{-1}}$ (which follows easily from the definition; cf. [R, Section 8]), the Hecke algebras on the right-hand side are identical. Therefore, we have an isomorphism

$$
\phi_{j}: \Psi_{G, \chi^{-1}}^{-1} \circ j \circ \Psi_{G, \chi}: \mathcal{H}(G, \rho) \longrightarrow \mathcal{H}\left(G, \rho^{-1}\right) .
$$

If $\left(\gamma, V_{\gamma}\right)$ is a representation of $\mathcal{H}(G, \rho)$, we let $\phi_{j}(\gamma)$ denote the representation of $\mathcal{H}\left(G, \rho^{-1}\right)$ associated to $\gamma$ by the Hecke algebra isomorphism above. Now, if $M$ is a standard Levi of $G$, let

$$
\begin{gathered}
E_{G, \chi}: R_{\chi}(G) \longrightarrow \mathcal{H}(G, \rho)-\bmod \\
E_{M, \chi}: R_{\chi}(M) \longrightarrow \mathcal{H}\left(M, \rho_{M}\right)-\bmod
\end{gathered}
$$

denote the functors giving the equivalence of categories. If $\pi \in \mathcal{R}_{\chi}(G)$, we define the Iwahori-Matsumoto involution of $\pi$ by

$$
j(\pi)=E_{G, \chi^{-1}}^{-1} \circ \phi_{j} \circ E_{G, \chi}(\pi) .
$$

Similarly, if $\tau \in \mathcal{R}_{\chi}(M)$, then $j(\tau)=E_{M, \chi^{-1}}^{-1} \circ \phi_{j} \circ E_{M, \chi}(\tau)$ (noting that $\phi_{j_{M}}=\left.\phi_{j}\right|_{\mathcal{H}\left(L, 1_{L}\right) \tilde{\otimes} \mathbb{C}\left[D_{\chi}\right]}$ from above).

\section{Theorem 1.8.}

$$
j\left(\operatorname{Ind}_{P}^{G} \tau\right) \cong \operatorname{Ind}_{P}^{G}(j(\tau))
$$


Proof. First, by Corollary 8.4 of $[\mathrm{Bu}-\mathbf{K}]$,

$$
E_{G, \chi}\left(\operatorname{Ind}_{P}^{G} \tau\right) \cong \operatorname{Ind}_{\mathcal{H}\left(M, \rho_{M}\right)}^{\mathcal{H}(G, \rho)}\left(E_{M, \chi}(\tau)\right)
$$

Therefore,

$$
\begin{aligned}
j\left(\operatorname{Ind}_{P}^{G} \tau\right) & \cong E_{G, \chi^{-1}}^{-1} \circ \phi_{j} \circ E_{G, \chi}\left(\operatorname{Ind}_{P}^{G} \tau\right) \\
& \cong E_{G, \chi^{-1}}^{-1} \circ \phi_{j}\left(\operatorname{Ind}_{\mathcal{H}\left(M, \rho_{M}\right)}^{\mathcal{H}(G, \rho)}\left(E_{M, \chi}(\tau)\right)\right) \\
& \cong E_{G, \chi^{-1}}^{-1}\left(\operatorname{Ind}_{\mathcal{H}\left(M, \rho_{M}^{-1}\right)}^{\mathcal{H}\left(G, \rho^{-1}\right)} \phi_{j}\left(E_{M, \chi}(\tau)\right)\right),
\end{aligned}
$$

by Lemma 1.7 above. By definition, $j(\tau)=E_{M, \chi^{-1}}^{-1} \circ \phi_{j} \circ E_{M, \chi}(\tau)$, or $E_{M, \chi^{-1}}(j(\tau))=\phi_{j}\left(E_{M, \chi}(\tau)\right)$. Thus,

$$
\begin{aligned}
j\left(\operatorname{Ind}_{P}^{G} \tau\right) & \cong E_{G, \chi^{-1}}\left(\operatorname{Ind}_{\mathcal{H}\left(M, \rho_{M}^{-1}\right)}^{\mathcal{H}\left(G, \rho^{-1}\right)} E_{M, \chi^{-1}} j(\tau)\right) \\
& \cong \operatorname{Ind}_{P}^{G}(j(\tau)),
\end{aligned}
$$

again, by Corollary 8.4 of $[\mathbf{B u}-\mathbf{K}]$.

We close by discussing a special case. First, let $G=G L(k)$ and $\mu$ a unitary character of $F^{\times}$. Consider the character

$$
\tau=|\cdot|^{\alpha+\frac{-k+1}{2}} \mu \otimes|\cdot|^{\alpha+\frac{-k+1}{2}+1} \mu \otimes \cdots \otimes|\cdot|^{\alpha+\frac{k-1}{2}} \mu
$$

of $T$. By definition,

$$
j(\tau)=|\cdot|^{-\alpha+\frac{k-1}{2}} \mu^{-1} \otimes|\cdot|^{-\alpha+\frac{k-1}{2}-1} \mu^{-1} \otimes \cdots \otimes|\cdot|^{-\alpha+\frac{-k+1}{2}} \mu^{-1} .
$$

Since $\left(\mu \circ \operatorname{det}_{k}\right)\left|\operatorname{det}_{k}\right|^{\alpha}$ is the unique irreducible subrepresentation of $\operatorname{Ind}_{B}^{G} \tau$, we see that $j\left(\left(\mu \circ \operatorname{det}_{k}\right)\left|\operatorname{det}_{k}\right|^{\alpha}\right)$ must be the unique irreducible subrepresentation of $\operatorname{Ind}_{B}^{G}(j(\tau))$, i.e.,

$$
j\left(\left(\mu \circ \operatorname{det}_{k}\right)\left|\operatorname{det}_{k}\right|^{\alpha}\right)=\left(\mu^{-1} \circ \operatorname{det}_{k}\right)\left|\operatorname{det}_{k}\right|^{-\alpha} \otimes S t_{k} .
$$

With this in hand, we may easily obtain the following:

Corollary 1.9. Let $G=S O(2 n+1)$ (resp., $G=S p(2 n)), P=M U a$ standard parabolic subgroup with $M \cong G L(k) \times S O(2(n-k)+1)($ resp., $M \cong$ $G L(k) \times S p(2(n-k))), \mu$ a unitary character of $F^{\times}$, and $\pi$ a representation of $S O(2(n-k)+1)$ (resp., $S p(2(n-k)))$. Then,

$$
j\left(\operatorname{Ind}_{P}^{G}\left(\left(\mu \circ \operatorname{det}_{k}\right)\left|\operatorname{det}_{k}\right|^{\alpha} \otimes \pi\right)\right) \cong \operatorname{Ind}_{P}^{G}\left(\left(\mu^{-1} \circ \operatorname{det}_{k}\right)\left|\operatorname{det}_{k}\right|^{-1} S t_{k} \otimes j(\pi)\right) .
$$




\section{The trivial character case; summary of Møglin's result.}

We recall Mœglin's results [M1]. All theorems in this section are due to Mœglin. Let $G(n)=S p(2 n), S O(2 n+1), O(2 n)$. Then the dual group is $G^{*}(n)=O(2 n+1, \mathbb{C}), S p(2 n, \mathbb{C}), O(2 n, \mathbb{C})$. Given a unipotent orbit $O \in$ $O(2 n+1, \mathbb{C}), S p(2 n, \mathbb{C})$, or $O(2 n, \mathbb{C})$, Mœglin formed a set $P(O)$ of ordered partitions as follows:

$\mathfrak{p}=\left(p_{1}, \ldots, p_{r} ; q_{1}, \ldots, q_{s}\right) \in P(O)$ if and only if:

(1) $\left(p_{1}, p_{1}, \ldots, p_{r}, p_{r}, q_{1}, \ldots, q_{s}\right)$ is $O$ if we ignore the ordering.

(2) $q_{i}$ are distinct and odd integers in the case of $O_{2 n+1}(\mathbb{C})$ and $O_{2 n}(\mathbb{C})$, even integers in the case of $S p_{2 n}(\mathbb{C})$.

(3) For all $1 \leq j \leq\left[\frac{s+1}{2}\right], q_{2 j-1}>q_{2 j}$ and there does not exist $1 \leq k \leq$ $\left[\frac{s+1}{2}\right]$ such that $q_{2 j-1}>q_{2 k-1}>q_{2 j}>q_{2 k}$.

(4) If there exists a $1 \leq k \leq s$ such that $q_{2 j-1}>q_{k}>q_{2 j}$, then $k<2 j-1$. We set $q_{s+1}=0$ if $s$ is odd. We can put an equivalence relation on $P(O)$ as follows: For $\mathfrak{p}=\left(p_{1}, \ldots, p_{r} ; q_{1}, \ldots, q_{s}\right), \mathfrak{p}^{\prime}=\left(p_{1}^{\prime}, \ldots, p_{r}^{\prime} ; q_{1}^{\prime}, \ldots, q_{s}^{\prime}\right) \in P(O)$, $\mathfrak{p} \simeq \mathfrak{p}^{\prime}$ if and only if for all $1 \leq i \leq\left[\frac{s+1}{2}\right]$, there exists $1 \leq j \leq\left[\frac{s+1}{2}\right]$ such that $q_{2 i-1}=q_{2 j-1}^{\prime}, q_{2 i}=q_{2 j}^{\prime}$. We note that $\left\{p_{1}, \ldots, p_{r}\right\}=\left\{p_{1}^{\prime}, \ldots, p_{r}^{\prime}\right\}$ as sets.

Remark 2.1. For a distinguished unipotent orbit, we have $r=0$. In that case, we write $\mathfrak{p}=\left(; q_{1}, \ldots, q_{s}\right)$.

Example 2.1. For a unipotent orbit of the form $(5,3,1)$ in $O_{9}(\mathbb{C})$, there are two non-equivalent elements in $P(O)$, namely, $(; 5,3,1)$ and $(; 3,1,5)$.

Remark 2.2. The conditions (3), (4) in $P(O)$ are such that the condition in [M1, Cor 0.10.2] is satisfied, and hence implies non-vanishing of normalized intertwining operators. We use this in Proposition 2.5. In that case, $x=$ $\frac{q_{1}-1}{2}, \frac{q_{1}-3}{2}, \ldots, \frac{q_{2}+1}{2}$ and $X$ is a sub-module of

$$
\operatorname{Ind}_{M} 1 \times||^{\frac{q_{3}-q_{4}}{4}} \times \cdots \times|| \frac{q_{2\left[\frac{s+1}{2}\right]-1}-q_{2\left[\frac{s+1}{2}\right]}}{4},
$$

where $M=G L\left(q_{2}\right) \times G L\left(\frac{q_{3}+q_{4}}{2}\right) \times \cdots \times G L\left(\frac{q_{2\left[\frac{s+1}{2}\right]-1}+q_{2\left[\frac{s+1}{2}\right]}}{2}\right)$.

For $\mathfrak{p}=\left(p_{1}, \ldots, p_{r} ; q_{1}, \ldots, q_{s}\right)$, we set, for $2 \leq i \leq r, p_{i}^{\prime}=p_{1}+\cdots+p_{i-1}$ and $p_{1}^{\prime}=0$ and for $1 \leq i \leq\left[\frac{s+1}{2}\right]$,

$$
\begin{aligned}
& T_{i}^{d}=\sum_{j=1}^{r} p_{j}+\sum_{1 \leq l<i} \frac{q_{2 l-1}+q_{2 l}}{2}, \\
& T_{i}^{f}=\sum_{j=1}^{r} p_{j}+\sum_{1 \leq l \leq i} \frac{q_{2 l-1}+q_{2 l}}{2} .
\end{aligned}
$$


We recall the definition of $\lambda_{\mathfrak{p}}$ and $w_{\mathfrak{p}}: \lambda_{\mathfrak{p}}=\left(\lambda_{\mathfrak{p}, 1}, \ldots, \lambda_{\mathfrak{p}, n}\right)$, where

$$
\begin{aligned}
\lambda_{\mathfrak{p}, p_{i}^{\prime}+t} & =\frac{p_{i}+1}{2}-t, \text { for } 1 \leq i \leq r \text { and } 1 \leq t \leq p_{i}, \\
\lambda_{\mathfrak{p}, T_{k}^{d}+t} & =\frac{q_{2 k-1}+1}{2}-t, \text { for } 1 \leq k \leq\left[\frac{s+1}{2}\right] \text { and } 1 \leq t \leq \frac{q_{2 k_{1}}+q_{2 k}}{2} .
\end{aligned}
$$

$w_{\mathfrak{p}}$ is an element of the Weyl group given by:

$$
\begin{aligned}
& w_{\mathfrak{p}}\left(p_{i}^{\prime}+t\right)=p_{i+1}^{\prime}-t+1, \text { for } 1 \leq i \leq r \text { and } 1 \leq t \leq p_{i} \\
& w_{\mathfrak{p}}(t)=-t, \text { for } 1 \leq k \leq\left[\frac{s+1}{2}\right] \text { and } T_{k}^{d}<t \leq T_{k}^{d}+\frac{q_{2 k_{1}}-q_{2 k}}{2}, \\
& w_{\mathfrak{p}}\left(T_{k}^{d}+\frac{q_{2 k-1}-q_{2 k}}{2}+t\right)=T_{k}^{f}-t+1, \\
& \text { for } 1 \leq k \leq\left[\frac{s+1}{2}\right] \text { and } 1 \leq t \leq q_{2 k} .
\end{aligned}
$$

Remark 2.3. All $\lambda_{\mathfrak{p}}$ are conjugates and $w_{\mathfrak{p}}^{2}=1$. Let $\lambda_{O}$ be the conjugate of $\lambda_{\mathfrak{p}}$ which is in the closure of the positive Weyl chamber.

We also define $\sigma_{p_{i}}$ for $1 \leq i \leq r$ and $\sigma_{k}$ for $1 \leq k \leq\left[\frac{s}{2}\right]$ and let $\operatorname{Stab}\left(\lambda_{\mathfrak{p}}, \uparrow\right.$ $\mathfrak{p})$ be the subgroup of $\operatorname{Stab}\left(\lambda_{\mathfrak{p}}\right)$ generated by these elements:

$$
\begin{aligned}
\sigma_{p_{i}}(j) & =j, \text { if } j \notin\left[p_{i}^{\prime}+1, p_{i+1}^{\prime}\right], \\
\sigma_{p_{i}}\left(p_{i}^{\prime}+t\right) & =-\left(p_{i+1}^{\prime}-t+1\right), \text { if } t \in\left[1, p_{i}\right], \\
\sigma_{k}(j) & =j, \text { if } j \notin\left[T_{k}^{d}+\frac{q_{2 k-1}-q_{2 k}}{2}+1, T_{k}^{f}\right], \\
\sigma_{k}\left(T_{k}^{d}+\frac{q_{2 k-1}-q_{2 k}}{2}+t\right) & =-\left(T_{k}^{f}-t+1\right), \text { if } t \in\left[1, q_{2 k}\right] .
\end{aligned}
$$

Let $A(O)$ be a finite abelian group generated by the order two elements $\sigma\left(p_{1}\right), \ldots, \sigma\left(p_{r}\right), \sigma\left(q_{1}\right), \ldots, \sigma\left(q_{s}\right)$. (We take only the distinct ones.) Let $\bar{A}(\mathfrak{p})=A(O) / K_{\mathfrak{p}}$, where $K_{\mathfrak{p}}$ is generated by $\sigma\left(q_{2 i-1}\right) \sigma\left(q_{2 i}\right)^{-1}$ for all $1 \leq i \leq$ $\left[\frac{s+1}{2}\right]$. We set $\sigma\left(q_{s+1}\right)=1$ if $s$ is odd.

Lemma 2.1. (1) $|\bar{A}(\mathfrak{p})|=2^{\left[\frac{s}{2}\right]}$.

(2) $\bar{A}(\mathfrak{p})$ is isomorphic to the quotient of $\operatorname{Stab}\left(\lambda_{\mathfrak{p}}, \uparrow \mathfrak{p}\right)$ by the subgroup generated by $\sigma\left(p_{i}\right) \sigma\left(p_{j}\right)^{-1}$ for $p_{i}=p_{j}$ and $\sigma\left(p_{i}\right) \sigma_{k}^{-1}$ for $p_{i}=q_{2 k-1}$ or $p_{i}=q_{2 k}$. The homomorphism $\operatorname{Stab}\left(\lambda_{\mathfrak{p}}, \uparrow \mathfrak{p}\right) \longmapsto \bar{A}(\mathfrak{p})$ is given by $\sigma\left(p_{i}\right) \longmapsto \sigma\left(p_{i}\right) K_{\mathfrak{p}}$ for $i=1, \ldots, r$ and $\sigma_{k} \longmapsto \sigma\left(q_{2 k-1}\right) K_{\mathfrak{p}}$.

Let Springer $(O)$ is the set of characters of $A(O)$ which is in the image of the Springer correspondence. We recall that the Springer correspondence is a one to one map from the set of characters of $W$, the Weyl group of $G^{*}$ into the set of pairs $(O, \eta)$, where $O$ is a unipotent orbit in $G^{*}$ and $\eta$ is a character of $A(O)$. Then: 
Theorem 2.2. Springer $(O) \simeq \cup_{\mathfrak{p} \in P(O)} \widehat{\overline{A(\mathfrak{p}})}$, where $\widehat{\overline{A(\mathfrak{p})}}$ is the character group of $\bar{A}(\mathfrak{p})$.

2.1. Local theory. Let $I\left(\lambda_{\mathfrak{p}}\right)=\operatorname{Ind}_{B}^{G} \exp \left(\left\langle\lambda_{\mathfrak{p}}, H_{B}()\right\rangle\right)$ (normalized induction). The normalized intertwining operator $R\left(w_{\mathfrak{p}}, \lambda\right)$ is not holomorphic at $\lambda_{\mathfrak{p}}$ in general. Mœglin defined $R\left(w_{\mathfrak{p}}, \lambda_{\mathfrak{p}}\right)$ as composition of several operators. (See $[\mathbf{M} 1]$ or Section 3) Then we have:

Theorem 2.3. (1) $R\left(w_{\mathfrak{p}}, \lambda_{\mathfrak{p}}\right) I\left(\lambda_{\mathfrak{p}}\right)$ is a direct sum of $|\bar{A}(\mathfrak{p})|$ irreducible representations with multiplicity 1 . Let $\operatorname{Unip}(\mathfrak{p})$ be the set of the irreducible direct summands and $\operatorname{Unip}(O)=\cup_{\mathfrak{p} \in P(O)} \operatorname{Unip}(\mathfrak{p})$. Then the Iwahori-Matsumoto involution of elements in $\operatorname{Unip}(O)$ is tempered.

(2) $\operatorname{Unip}(O)$ is exactly the set of irreducible representations of $G(n)$ whose infinitesimal character is $\lambda_{O}$ and whose Iwahori-Matsumoto involution is tempered.

(3) If $r=0$, i.e., $O$ is a distinguished unipotent orbit, then the IwahoriMatsumoto involution of elements in $\operatorname{Unip}(O)$ is square integrable.

Mœglin proves Theorem 2.3 by induction: Let $\mathfrak{p}=\left(p_{1}, \ldots, p_{r} ; q_{1}, \ldots, q_{s}\right)$. Suppose $r \neq 0$. Set $\mathfrak{p}^{\prime}=\left(p_{2}, \ldots, p_{r} ; q_{1}, \ldots, q_{s}\right)$. We have

$$
R\left(w_{\mathfrak{p}}, \lambda_{\mathfrak{p}}\right) I\left(\lambda_{\mathfrak{p}}\right)=\operatorname{Ind}_{G L\left(p_{1}\right) \times G\left(n-p_{1}\right)} 1 \times R\left(w_{\mathfrak{p}^{\prime}}, \lambda_{\mathfrak{p}^{\prime}}\right) I\left(\lambda_{\mathfrak{p}^{\prime}}\right) .
$$

We denote by $j$ the Iwahori-Matsumoto involution. Then

$$
j R\left(w_{\mathfrak{p}}, \lambda_{\mathfrak{p}}\right) I\left(\lambda_{\mathfrak{p}}\right)=\operatorname{Ind}_{G L\left(p_{1}\right) \times G\left(n-p_{1}\right)} S t\left(p_{1}\right) \times j R\left(w_{\mathfrak{p}^{\prime}}, \lambda_{\mathfrak{p}^{\prime}}\right) I\left(\lambda_{\mathfrak{p}^{\prime}}\right),
$$

where $S t\left(p_{1}\right)$ is the Steinberg representation of $G L\left(p_{1}\right)$. By induction, $j R\left(w_{\mathfrak{p}^{\prime}}, \lambda_{\mathfrak{p}^{\prime}}\right) I\left(\lambda_{\mathfrak{p}^{\prime}}\right)$ is a semi-simple tempered representation of length $\left|\bar{A}\left(\mathfrak{p}^{\prime}\right)\right|$. Let $X^{\prime} \in \operatorname{Unip}\left(\mathfrak{p}^{\prime}\right)$.

Proposition 2.4. The induced representation

$$
\operatorname{Ind}_{G L\left(p_{1}\right) \times G\left(n-p_{1}\right)} S t\left(p_{1}\right) \times j X^{\prime},
$$

i.e., $\operatorname{Ind}_{G L\left(p_{1}\right) \times G\left(n-p_{1}\right)} 1 \times X^{\prime}$ is irreducible if and only if $p_{1}=p_{j}$ or $q_{k}$ for some $j=2, \ldots, r$ or $k=1, \ldots, s$. If it is reducible, then it is a sum of two irreducible representations.

For $x$ small, the normalized intertwining operator associated to $\sigma_{p_{1}}$ is

$$
\begin{aligned}
& \operatorname{Ind}_{G L(1) \times \cdots \times G L(1) \times G\left(n-p_{1}\right)}||^{\frac{p_{1}-1}{2}+x} \times \cdots \times||^{-\frac{p_{1}-1}{2}+x} \times X^{\prime} \\
& \longrightarrow \operatorname{Ind}_{G L(1) \times \cdots \times G L(1) \times G\left(n-p_{1}\right)}||^{-\frac{p_{1}-1}{2}-x} \times \cdots \times||^{\frac{p_{1}-1}{2}-x} \times X^{\prime} .
\end{aligned}
$$

This operator is a product of the operator

$$
\begin{aligned}
\operatorname{Ind}_{G L(1) \times \cdots \times G L(1) \times G\left(n-p_{1}\right)}||^{\frac{p_{1}-1}{2}+x} & \times \cdots \times||^{-\frac{p_{1}-1}{2}+x} \times X^{\prime} \\
& \longrightarrow \operatorname{Ind}_{G L\left(p_{1}\right) \times G\left(n-p_{1}\right)}|\operatorname{det}|^{x} \times X^{\prime},
\end{aligned}
$$


and the operator, $R_{X^{\prime}}\left(\sigma_{p_{1}}, x\right)$ :

$$
\operatorname{Ind}_{G L\left(p_{1}\right) \times G\left(n-p_{1}\right)}|\operatorname{det}|^{x} \times X^{\prime} \longmapsto \operatorname{Ind}_{G L\left(p_{1}\right) \times G\left(n-p_{1}\right)}|\operatorname{det}|^{-x} \times X^{\prime} .
$$

Proposition 2.5. $R_{X^{\prime}}\left(\sigma_{p_{1}}, x\right)$ is holomorphic at $x=0$. Let $R_{X^{\prime}}\left(\sigma_{p_{1}}, 0\right)=$ $R_{X^{\prime}}\left(\sigma_{p_{1}}\right)$. Then $R_{X^{\prime}}\left(\sigma_{p_{1}}\right)^{2}=\mathrm{id}$ and $R_{X^{\prime}}\left(\sigma_{p_{1}}\right)$ is the identity if and only if $\operatorname{Ind}_{G L\left(p_{1}\right) \times G\left(n-p_{1}\right)} 1 \times X^{\prime}$ is irreducible. Let $R\left(\sigma_{p_{1}}\right)$ be the sum of $R_{X^{\prime}}\left(\sigma_{p_{1}}\right)$ as $X^{\prime}$ runs through $\operatorname{Unip}\left(\mathfrak{p}^{\prime}\right)$. Then it defines an intertwining operator for $R\left(w_{\mathfrak{p}}, \lambda_{\mathfrak{p}}\right) I\left(\lambda_{\mathfrak{p}}\right)$.

In a similar way, we can define $R\left(\sigma_{p_{i}}\right)$.

Suppose $r=0$. From [M1, 676] or Section 3, we know that

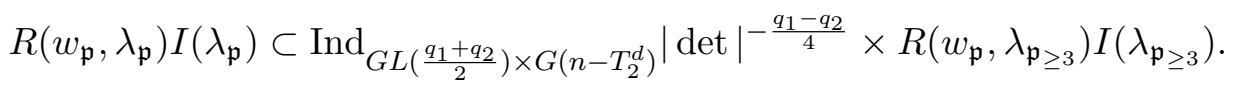

We use induction: Let $Y \in \operatorname{Unip}\left(\mathfrak{p}_{\geq 3}\right)$ and consider

$$
W_{Y}=\operatorname{Ind}_{G L\left(\frac{q_{1}+q_{2}}{2}\right) \times G\left(n-T_{2}^{d}\right)}|\operatorname{det}|^{\frac{q_{1}-q_{2}}{4}} \times Y .
$$

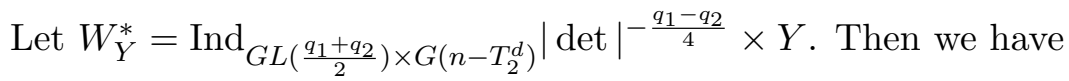

$$
j W_{Y}^{*}=\operatorname{Ind}_{G L\left(\frac{q_{1}+q_{2}}{2}\right) \times G\left(n-T_{2}^{d}\right)} S t\left(\frac{q_{1}+q_{2}}{2}\right) \otimes|\operatorname{det}|^{\frac{q_{1}-q_{2}}{4}} \times j Y .
$$

Proposition 2.6. $j W_{Y}^{*}$ is reducible and its subrepresentations are tempered.

Consider the following commutative diagram:

$$
\begin{aligned}
& Z_{Y}=\operatorname{Ind}_{G L(1) \times \cdots \times G L(1) \times G L\left(q_{2}\right) \times G\left(n-\frac{q_{1}+q_{2}}{2}\right)}||^{\frac{q_{1}-1}{2}} \longrightarrow W_{Y} \\
& \times||^{\frac{q_{1}-3}{2}} \times \cdots \times||^{\frac{q_{2}+1}{2}} \times 1 \times Y \\
& R_{Y}^{\prime} \downarrow \\
& Z_{Y}^{*}=\operatorname{Ind}_{G L(1) \times \cdots \times G L(1) \times G L\left(q_{1}\right) \times G\left(n-\frac{q_{1}+q_{2}}{2}\right)}||^{-\frac{q_{1}-1}{2}} \\
& \times||^{-\frac{q_{1}-3}{2}} \times \cdots \times\left.\right|^{-\frac{q_{2}+1}{2}} \times 1 \times Y
\end{aligned}
$$

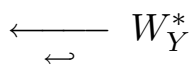

where $R_{Y}^{\prime}$ is the normalized intertwining operator. Its image is $R\left(w_{\mathfrak{p}}, \lambda_{\mathfrak{p}}\right) I\left(\lambda_{\mathfrak{p}}\right) \cap Z_{Y}^{*}$. By Proposition 2.4, $\operatorname{Ind}_{G L\left(q_{2}\right) \times G\left(n-\frac{q_{1}+q_{2}}{2}\right)} 1 \times Y$ is semisimple with length 2 .

Proposition 2.7. The image of $R_{Y}^{\prime}$ is semi-simple with length $\leq 2$.

Proof. Let $X$ be a subrepresentation of $Z_{Y}^{*}$. Let $\xi=||^{-\frac{q_{1}-1}{2}} \times||^{-\frac{q_{1}-3}{2}} \times$ $\cdots \times||^{-\frac{q_{2}+1}{2}}$. Consider the subrepresentation of the Jacquet module of $X$ with respect to $M=\underbrace{G L(1) \times \cdots \times G L(1)} \times G\left(n-\frac{q_{1}-q_{2}}{2}\right)$ such that the $\frac{q_{1}-q_{2}}{2}$ 
copies of $G L(1)$ acts, after semi-simplification, according to $\xi$, i.e., the space of generalized weight $\xi$. We denote the space by $\operatorname{Jac}_{\xi} X$. Then we have

$$
\operatorname{Jac}_{\xi} X \subset \operatorname{Jac}_{\xi} Z_{Y}^{*}=\xi \times \operatorname{Ind}_{G L\left(q_{2}\right) \times G\left(n-\frac{q_{1}+q_{2}}{2}\right)} 1 \times Y .
$$

Therefore our assertion follows.

Let Ind $_{G L\left(q_{2}\right) \times G\left(n-\frac{q_{1}+q_{2}}{2}\right)} 1 \times Y=X_{1}+X_{2}$. Then by [M1, Cor. 0.10.2], the normalized intertwining operators

$$
\begin{aligned}
& \operatorname{Ind}_{G L(1) \times \cdots \times G L(1) \times G\left(n-\frac{q_{1}-q_{2}}{2}\right)}||^{\frac{q_{1}-1}{2}} \times||^{\frac{q_{1}-3}{2}} \times \cdots \times||^{\frac{q_{2}+1}{2}} \times X_{i} \\
& \longrightarrow \operatorname{Ind}_{G L(1) \times \cdots \times G L(1) \times G\left(n-\frac{q_{1}-q_{2}}{2}\right)}||^{-\frac{q_{1}-1}{2}} \times||^{-\frac{q_{1}-3}{2}} \times \cdots \times||^{-\frac{q_{2}+1}{2}} \times X_{i}
\end{aligned}
$$

are non-vanishing. Therefore:

Proposition 2.8. The image of $R_{Y}^{\prime}$ is a sum of two irreducible representations.

Proposition 2.9. Let $R_{Y}\left(\sigma_{1}, x\right)$ be the operator:

$$
\operatorname{Ind}_{G L\left(q_{2}\right) \times G\left(n-\frac{q_{1}+q_{2}}{2}\right)}|\operatorname{det}|^{x} \times Y \longrightarrow \operatorname{Ind}_{G L\left(q_{2}\right) \times G\left(n-\frac{q_{1}+q_{2}}{2}\right)}|\operatorname{det}|^{-x} \times Y .
$$

$R_{Y}\left(\sigma_{1}, x\right)$ is holomorphic at $x=0$ and let $R_{Y}\left(\sigma_{1}, 0\right)=R_{Y}\left(\sigma_{1}\right)$. Then $R_{Y}\left(\sigma_{1}\right)^{2}=\mathrm{id}$ and $R_{Y}\left(\sigma_{1}\right)$ is not the identity since $\operatorname{Ind}_{G L\left(q_{2}\right) \times G\left(n-\frac{q_{1}+q_{2}}{2}\right)} 1 \times Y$ is reducible. Let $R\left(\sigma_{1}\right)$ be the sum of $R_{Y}\left(\sigma_{1}\right)$ as $Y$ runs through $\operatorname{Unip}\left(\mathfrak{p}_{\geq 2}\right)$. Then it defines an intertwining operator for $R\left(w_{\mathfrak{p}}, \lambda_{\mathfrak{p}}\right) I\left(\lambda_{\mathfrak{p}}\right)$.

In the same way, we can define $R\left(\sigma_{k}\right)$. For each $\sigma \in \operatorname{Stab}\left(\lambda_{\mathfrak{p}}, \uparrow \mathfrak{p}\right)$, we define $R(\sigma)$ in a canonical way $([\mathbf{M} 1,686])$ : If $\sigma=\sigma_{p_{1}} \cdots \sigma_{p_{r}} \sigma_{1} \cdots \sigma_{k}$, then $R(\sigma)=R\left(\sigma_{p_{1}}\right) \cdots R\left(\sigma_{p_{r}}\right) R\left(\sigma_{1}\right) \cdots R\left(\sigma_{k}\right)$. We note that $R\left(\sigma w_{\mathfrak{p}}, \lambda_{\mathfrak{p}}\right)=$ $R(\sigma) R\left(w_{\mathfrak{p}}, \lambda_{\mathfrak{p}}\right)$ for $\sigma \in \operatorname{Stab}\left(\lambda_{\mathfrak{p}}, \uparrow \mathfrak{p}\right)$.

\section{Theorem 2.10.}

(1) $\sigma \longmapsto R(\sigma)$ is a homomorphism of the group $\operatorname{Stab}\left(\lambda_{\mathfrak{p}}, \uparrow \mathfrak{p}\right)$ into the group of the intertwining operators of $R\left(w_{\mathfrak{p}}, \lambda_{\mathfrak{p}}\right) I\left(\lambda_{\mathfrak{p}}\right)$.

(2) For $X \in \operatorname{Unip}(\mathfrak{p})$, let $R(\sigma) X=\eta_{X}^{\mathfrak{p}}(\sigma) X$. Then $\eta_{X}^{\mathfrak{p}}$ defines a character of $\operatorname{Stab}\left(\lambda_{\mathfrak{p}}, \uparrow \mathfrak{p}\right)$.

(3) If $p_{i}=p_{j}$, then $\eta_{X}^{\mathfrak{p}}\left(\sigma\left(p_{i}\right)\right)=\eta_{X}^{\mathfrak{p}}\left(\sigma\left(p_{j}\right)\right)$. If $p_{i}=q_{2 k-1}$ or $p_{i}=q_{2 k}$, then $\eta_{X}^{\mathfrak{p}}\left(\sigma\left(p_{i}\right)\right)=\eta_{X}^{\mathfrak{p}}\left(\sigma_{k}\right)$. Recall in Proposition 2.4 that these happen precisely when $\operatorname{Ind}_{G L\left(p_{i}\right) \times G(m)} 1 \times X^{\prime}$ is irreducible for an appropriate $m$. Therefore by Lemma 2.1, $\eta_{X}^{\mathfrak{p}}$ defines a character of $\bar{A}(\mathfrak{p})$.

(4) By Proposition 2.5 and 2.9, passing to quotient, $X \longmapsto \eta_{X}^{\mathfrak{p}}$ gives rise to an isomorphism $\operatorname{Unip}(\mathfrak{p}) \simeq \widehat{\overline{A(p)}}$ which is extended canonically to

$$
\operatorname{Unip}(O) \simeq \operatorname{Springer}(O),
$$


by $X \longmapsto \eta_{X}$ in the sense that $\left|\operatorname{Unip}(\mathfrak{p}) \cap \operatorname{Unip}\left(\mathfrak{p}^{\prime}\right)\right|=\left|\widehat{\overline{A(\mathfrak{p})}} \cap \widehat{\overline{A\left(\mathfrak{p}^{\prime}\right)}}\right|$ and for $X \in \operatorname{Unip}(\mathfrak{p}) \cap \operatorname{Unip}\left(\mathfrak{p}^{\prime}\right), \eta_{X}^{\mathfrak{p}}=\eta_{X}^{\mathfrak{p}^{\prime}}$.

(5) If $\mathfrak{p} \simeq \mathfrak{p}^{\prime}$, then $\operatorname{Unip}(\mathfrak{p}) \simeq \widehat{\overline{A(\mathfrak{p})}}=\widehat{\overline{A\left(\mathfrak{p}^{\prime}\right)}} \simeq \operatorname{Unip}\left(\mathfrak{p}^{\prime}\right)$. In other words, up to isomorphism, $\operatorname{Unip}(\mathfrak{p})$ depends only on the equivalence class of $\mathfrak{p}$.

Especially, if $\mathfrak{p}=\left(; q_{1}, \ldots, q_{s}\right) \in P(O), O$ a distinguished unipotent orbit, then we have:

Corollary 2.11. (1) $\bar{A}(\mathfrak{p})$ is isomorphic to $\operatorname{Stab}\left(\lambda_{\mathfrak{p}}, \uparrow \mathfrak{p}\right)$.

(2) $\sigma \longmapsto R(\sigma)$ is an isomorphism of the group $\operatorname{Stab}\left(\lambda_{\mathfrak{p}}, \uparrow \mathfrak{p}\right)$ onto the group of the intertwining operators of $R\left(w_{\mathfrak{p}}, \lambda_{\mathfrak{p}}\right) I\left(\lambda_{\mathfrak{p}}\right)$.

We think of $\eta \in \widehat{\overline{A(\mathfrak{p})}}$ as an element of $\widehat{A(O)}$ such that $\left.\eta\right|_{K_{\mathfrak{p}}}=1$.

2.2. Global Theory. Let $O$ be a unipotent orbit and $\mathfrak{p}=\left(p_{1}, \ldots, p_{r}\right.$; $\left.q_{1}, q_{2}, \ldots, q_{s}\right) \in P(O)$. Let $S_{\mathfrak{p}}$ be the set of positive roots defined as follows:

$$
\begin{cases}e_{j}-e_{j+1}, & \text { for } \sum_{i=1}^{k} p_{i}<j<\sum_{i=1}^{k+1} p_{i}, \\ e_{j}-e_{j+1}, & \text { for } T_{i}^{d}<j \leq T_{i}^{f}-1 \text { and } \\ & e_{T_{i}^{d}+\frac{q_{2 i}-q_{2 i-1}}{2}+e_{T_{i}^{f}}, \text { where } 1 \leq i \leq\left[\frac{s}{2}\right],} \\ e_{j}-e_{j+1}, & \text { for } T_{\frac{s+1}{2}}^{d}<j<n \\ 2 e_{n}, & \text { if } G=S p(2 n), s \text { is odd and } q_{s}>1 \\ e_{n}, & \text { if } G=S O(2 n+1), s \text { is odd. }\end{cases}
$$

We note that $S_{\mathfrak{p}} \subset\left\{\alpha>0 \mid w_{\mathfrak{p}} \alpha<0,\left\langle\lambda_{\mathfrak{p}}, \alpha^{\vee}\right\rangle=1\right\}$ and $S_{\mathfrak{p}}$ has exactly $n-r$ elements. We will take the iterated residue of the Eisenstein series along the $n$ singular hyperplanes $\left\langle\lambda_{\mathfrak{p}}, \alpha^{\vee}\right\rangle=1$ for $\alpha \in S_{\mathfrak{p}}$.

Definition 2.2.1. For $\mathfrak{p}=\left(p_{1}, \ldots, p_{r} ; q_{1}, q_{2}, \ldots, q_{s}\right) \in P(O)$, we define

$$
\begin{aligned}
M_{\mathfrak{p}}=G L\left(p_{1}\right) \times \cdots \times & G L\left(p_{r}\right) \times G\left(n-T_{1}^{d}\right), \\
M_{\mathfrak{p}}^{\prime}=G L\left(p_{1}\right) \times \cdots \times & G L\left(p_{r}\right) \times G L\left(\frac{q_{1}+q_{2}}{2}\right) \times \cdots \\
& \times G L\left(\frac{q_{2\left[\frac{s+1}{2}\right]-1}+q_{2\left[\frac{s+1}{2}\right]}}{2}\right) .
\end{aligned}
$$

If $s$ is odd, we put the convention that $\frac{q_{2\left[\frac{s+1}{2}\right]-1}+q_{2\left[\frac{s+1}{2}\right]}}{2}$ is $\frac{q_{s}-1}{2}$ if $G=S p(2 n)$, and $\frac{q_{s}}{2}$ if $G=S O(2 n+1)$.

Definition 2.2.2. Let $V(\mathfrak{p})$ (resp. $V^{\prime}(\mathfrak{p})$ ) be the set of elements of the form $\lambda_{\mathfrak{p}}+\eta$, where $\eta$ is a character of $M_{\mathfrak{p}}(\mathbb{A})\left(\right.$ resp. $\left.M_{\mathfrak{p}}^{\prime}(\mathbb{A})\right)$. Note that if $r=0, V(\mathfrak{p})=\left\{\lambda_{\mathfrak{p}}\right\}$. We note that $V^{\prime}(\mathfrak{p})$ is the intersection of the singular hyperplanes $\left\langle\lambda, \alpha^{\vee}\right\rangle=1$, where $\alpha \in\left\{e_{j}-e_{j+1}\right.$ for $\sum_{i=1}^{k} p_{i}<j<\sum_{i=1}^{k+1} p_{i}$, $T_{i}^{d}<j \leq T_{i}^{f}-1, i=1, \ldots,\left[\frac{s}{2}\right]$ and $\left.T_{s}^{f}<j<n\right\}$. 
We denote the element in $V^{\prime}(\mathfrak{p})$ as

$$
\begin{aligned}
& \lambda_{\mathfrak{p}}\left(x_{1}, \ldots, x_{r}, z_{1}, \ldots, z_{\left[\frac{s+1}{2}\right]}\right) \\
& =\lambda_{\mathfrak{p}}+(\underbrace{x_{1}, \ldots, x_{1}}_{p_{1}}, \ldots, \underbrace{x_{r}, \ldots, x_{r}}_{p_{r}}, \underbrace{z_{1}, \ldots, z_{1}}_{\frac{q_{1}+q_{2}}{2}}, \ldots, \underbrace{2}_{\frac{q_{2\left[\frac{s+1}{2}\right]-1}+q_{2\left[\frac{s+1}{2}\right]}}{z_{\left[\frac{s+1}{2}\right]}, \ldots, z_{\left[\frac{s+1}{2}\right]}}} .
\end{aligned}
$$

Definition 2.2.3. For $1 \leq k \leq\left[\frac{s+1}{2}\right]$, we define

$$
\begin{aligned}
V_{k}^{\prime}(\mathfrak{p})=\left\{\lambda_{\mathfrak{p}}\left(x_{1}, \ldots, x_{r}, z_{1}, \ldots, z_{\left[\frac{s+1}{2}\right]}\right) \in\right. & V^{\prime}(\mathfrak{p}), \\
& \text { such that } \left.z_{i}=0 \text { for all } i>k\right\} .
\end{aligned}
$$

In particular, $V_{0}^{\prime}(\mathfrak{p})=V(\mathfrak{p})$ and $V_{\left[\frac{s+1}{2}\right]}^{\prime}=V^{\prime}(\mathfrak{p})$.

Definition 2.2.4. We define $W(\uparrow, \mathfrak{p})$ to be the set of the Weyl group elements which send the positive roots of $M_{\mathfrak{p}}^{\prime}$ to the positive roots of $M_{\mathfrak{p}}^{\prime}$.

Let

$$
d(\mathfrak{p}, \lambda)=\prod_{\alpha \in S_{\mathfrak{p}}}\left(\left\langle\lambda_{\mathfrak{p}}, \alpha^{\vee}\right\rangle-1\right) .
$$

Let Unip be the submodule of $\otimes_{v}^{\prime} R_{v}\left(w_{\mathfrak{p}}, \lambda_{\mathfrak{p}}\right) I_{v}\left(\lambda_{\mathfrak{p}}\right)$ which is the sum of irreducible subrepresentations of type $\otimes_{v}^{\prime} X_{v}$, where $X_{v} \in \operatorname{Unip}(\mathfrak{p})$ for all $v$ and there does not exist $\mathfrak{p}^{\prime}>\mathfrak{p}$ and $X_{v} \in \operatorname{Unip}\left(\mathfrak{p}^{\prime}\right)$ for all $v$.

Let $\operatorname{proj}_{[\mathfrak{p}]}$ be the $G(\mathbb{A})$-projection $\otimes_{v}^{\prime} R_{v}\left(w_{\mathfrak{p}}, \lambda_{\mathfrak{p}}\right) I_{v}\left(\lambda_{\mathfrak{p}}\right) \longmapsto$ Unip. For $\phi \in P W$, the set of Paley-Wiener type functions, let

$$
l_{\mathfrak{p}}(\phi, \lambda)=\sum_{w \in W} r(w,-\lambda) R\left(w_{\mathfrak{p}} w^{-1}, w \lambda\right) \phi(w \lambda) .
$$

Then we have:

(1) $r\left(w_{\mathfrak{p}}, \lambda\right) d(\mathfrak{p}, \lambda)$ is holomorphic at $\lambda=\lambda_{\mathfrak{p}}$ and its value is non-zero.

(2) The poles of $l_{\mathfrak{p}}(\phi, \lambda)$ in a neighborhood of $\lambda_{\mathfrak{p}}$ are contained in the local intertwining operators.

(3) $r(w,-\lambda)$ is identically zero on $V^{\prime}(\mathfrak{p})$ if $w \notin W(\uparrow, \mathfrak{p})$. So the restriction of $l_{\mathfrak{p}}(\phi, \lambda)$ to $V^{\prime}(\mathfrak{p})$ is given by

$$
l_{\mathfrak{p}}(\phi, \lambda)=\sum_{w \in W(\uparrow, \mathfrak{p})} r(w,-\lambda) R\left(w_{\mathfrak{p}} w^{-1}, w \lambda\right) \phi(w \lambda) .
$$

(4) $l_{\mathfrak{p}}(\phi, \lambda)_{V(\mathfrak{p})}$ can be defined inductively by restricting it to $V_{k}^{\prime}(\mathfrak{p})$ from $k=\left[\frac{s+1}{2}\right]-1$ to $k=0$.

(5) $l_{\mathfrak{p}}(\phi, \lambda)_{V(\mathfrak{p})}$ is holomorphic at $\lambda_{\mathfrak{p}}$ and $l_{\mathfrak{p}}\left(\phi, \lambda_{\mathfrak{p}}\right) \in \otimes_{v}^{\prime} R_{v}\left(w_{\mathfrak{p}}, \lambda_{\mathfrak{p}}\right) I_{v}\left(\lambda_{\mathfrak{p}}\right)$. This depends only on $\phi$ and the equivalence class of $\mathfrak{p}$. Let $l_{[\mathfrak{p}]}\left(\phi, \lambda_{\mathfrak{p}}\right)=$ $\operatorname{proj}_{[\mathfrak{p}]}\left(\phi, \lambda_{\mathfrak{p}}\right)$. 
(6) Let $\langle$,$\rangle be the inner product in L^{2}(G(F) \backslash G(\mathbb{A}))$. Then

$$
\begin{aligned}
\left\langle\theta_{\phi^{\prime}}, \theta_{\phi}\right\rangle=\lim _{T \rightarrow \infty} \sum_{O \subset G^{*}(n)} \sum_{\mathfrak{p} \in P(O)} c_{\mathfrak{p}} \int_{\lambda \in V(\mathfrak{p}), \operatorname{Re} \lambda=\lambda_{\mathfrak{p}},\|\operatorname{Im} \lambda\|^{2} \leq T} \\
\left\langle\left\langle l_{[\mathfrak{p}]}^{\prime}\left(\phi^{\prime}, \bar{\lambda}\right), l_{[\mathfrak{p}]}(\phi, \lambda)\right\rangle_{V(\mathfrak{p})}\left(r\left(w_{\mathfrak{p}}, \lambda\right) d(\mathfrak{p}, \lambda)\right)_{V(\mathfrak{p})},\right.
\end{aligned}
$$

where $O$ runs through the unipotent orbits in $G^{*}(n)$ and $\mathfrak{p}$ runs through the set of representatives in each equivalence classes in $P(O)$.

(7) For $\phi \in P W$, suppose $l_{[\mathfrak{p}]}\left(\phi, \lambda_{\mathfrak{p}}\right)$ generates an irreducible representation. Then for all $v$ finite places, let $X_{v}$ be the local representation of $G_{v}$ generated by $l_{[\mathfrak{p}]}\left(\phi, \lambda_{\mathfrak{p}}\right)$. Then $X_{v} \in \operatorname{Unip}(\mathfrak{p})$ and $\prod_{v} \eta_{X_{v}}=1$.

(8) Conversely, suppose $\mathfrak{p}=\left(p_{1}, \ldots, p_{r} ; q_{1}, \ldots, q_{s}\right) \in P(O)$ such that $p_{i} \neq p_{j}$ for $i, j \in[1, r]$ and $\pi=\otimes_{v}^{\prime} X_{v}$ is an irreducible automorphic representation which satisfies the following: (a) $X_{v} \in \operatorname{Unip}(\mathfrak{p})$ for all $v$, (b) $X_{v}$ is spherical almost everywhere and at archimedean places, and (c) $\prod_{v} \eta_{X_{v}}=1$. Then there exists $\phi \in P W$ such that the representation generated by $l_{[\mathfrak{p}]}\left(\phi, \lambda_{\mathfrak{p}}\right)$ is isomorphic to $\pi$.

(9) In fact, for an appropriate $\phi \in P W$,

$$
l_{\mathfrak{p}}\left(\phi, \lambda_{\mathfrak{p}}\right)=\sum_{\tau \in \operatorname{Stab}\left(\lambda_{\mathfrak{p}}, \uparrow \mathfrak{p}\right)} R\left(\tau^{-1}\right) R\left(w_{\mathfrak{p}}, \lambda_{\mathfrak{p}}\right) \phi\left(\lambda_{\mathfrak{p}}\right)
$$

\section{Arbitrary character case.}

By conjugation, we can assume $\chi=\chi(\underbrace{\mu_{1}, \ldots, \mu_{1}}_{r_{1}}, \ldots, \underbrace{\mu_{k}, \ldots, \mu_{k}}_{r_{k}}, \underbrace{1, \ldots, 1}_{r_{0}})$, $r_{0}+\cdots+r_{k}=n, r_{1} \geq \cdots \geq r_{k}, \mu_{i}$ 's are distinct local quadratic characters. Here $k \leq 3$ (Recall that we are dealing with a $p$-adic field with odd residual characteristic and hence there are only three non-trivial distinct quadratic characters.) Set $\mu_{0}=1$. We use the following notation throughout this section:

(1) If $G=S p(2 n), G^{\prime}=G_{1}^{\prime} \times \cdots \times G_{k}^{\prime} \times G_{0}^{\prime}$, where $G_{i}^{\prime}=O\left(2 r_{i}\right)$ for $i=1, \ldots, k, G_{0}^{\prime}=S p\left(2 r_{0}\right)$. Also we denote $G_{i}^{*}=O\left(2 r_{i}, \mathbb{C}\right)$ for $i=$ $1, \ldots, k, G_{0}^{*}=O\left(2 r_{0}+1, \mathbb{C}\right)$.

(2) If $G=S O(2 n+1), G^{\prime}=G_{1}^{\prime} \times \cdots \times G_{k}^{\prime} \times G_{0}^{\prime}$, where $G_{i}^{\prime}=S O\left(2 r_{i}+1\right)$ for $i=1, \ldots, k, G_{0}^{\prime}=S O\left(2 r_{0}+1\right)$. Also we denote $G_{i}^{*}=S p\left(2 r_{i}, \mathbb{C}\right)$ for $i=1, \ldots, k, G_{0}^{*}=S p\left(2 r_{0}, \mathbb{C}\right)$.

(3) If $G=O(2 n), G^{\prime}=G_{1}^{\prime} \times \cdots \times G_{k}^{\prime} \times G_{0}^{\prime}$, where $G_{i}^{\prime}=O\left(2 r_{i}\right)$ for $i=1, \ldots, k, G_{0}^{\prime}=O\left(2 r_{0}\right)$. Also we denote $G_{i}^{*}=O\left(2 r_{i}, \mathbb{C}\right)$ for $i=$ $1, \ldots, k, G_{0}^{*}=O\left(2 r_{0}, \mathbb{C}\right)$.

We need to first generalize Mœglin's results to an arbitrary chain. 
Definition 3.1. By a segment attached to $(a, b)$, we mean a descending sequence of characters

$$
\lambda_{(a, b)}=||^{\lambda_{1}} \times||^{\lambda_{2}} \times \cdots \times||^{\lambda_{n}},
$$

where $\lambda_{t}=\frac{a+1}{2}-t, n=\frac{a+b}{2}$. We sometimes write it as $\lambda_{(a, b)}=\left(\lambda_{1}, \ldots, \lambda_{n}\right)$. We put the convention that $a>b>0(a, b$ are odd in the case of $S p(2 n)$ and $O(2 n)$, even in the case of $S O(2 n+1))$. To $(a), a \geq 3$, we attach a segment

$$
\lambda_{(a)}=||^{\frac{a-1}{2}} \times||^{\frac{a-3}{2}} \times \cdots \times||^{1} .
$$

We write it as $\lambda_{(a)}=\left(\frac{a-1}{2}, \frac{a-3}{2}, \ldots, 1\right)$. By a chain we mean an ordered union of segments. We put a convention that the segment attached to $(a)$ appears at the end. It can appear only in the case of $S p(2 n)$ and $S O(2 n+1)$. A chain is denoted by $\mathfrak{p}=\left(a_{1}, b_{1}, a_{2}, b_{2}, \ldots, a_{s}, b_{s}, a_{s+1}\right)$, where $\left(a_{i}, b_{i}\right)$ is a segment and $\lambda_{\mathfrak{p}}=\lambda_{\left(a_{1}, b_{1}\right)} \cdots \lambda_{\left(a_{s}, b_{s}\right)} \lambda_{\left(a_{s+1}\right)}$ with an obvious meaning.

Remark 3.1. We note that $\lambda_{(a, b)}$ is the intersection of the $n$ singular hyperplanes $e_{1}-e_{2}=1, e_{2}-e_{3}=1, \ldots, e_{n-1}-e_{n}=1, e_{\frac{a-b}{2}}+e_{n}=1$.

Remark 3.2. Suppose we ignore the ordering in $\mathfrak{p}$. Then it corresponds to a unipotent orbit $O$ in $G^{*}(n)$. When $O$ is a distinguished orbit, Mœglin's case is that $a_{i}, b_{i}$ are all distinct and satisfy two additional conditions:

(1) there does not exist $1 \leq k \leq s$ such that $a_{i}>a_{k}>b_{i}>b_{k}$.

(2) if there exists $a_{k}$ or $b_{k}$ such that $a_{j}>a_{k}>b_{j}$ or $a_{j}>b_{k}>b_{j}$, then $k<j$.

Let $\mathfrak{p}=\left(a_{1}, b_{1}, \ldots, a_{s}, b_{s}, a_{s+1}\right)$. If $\left\{a_{i}, b_{i}\right\} \cap\left\{a_{i+1}, b_{i+1}\right\} \neq \emptyset$, then we could permute $\left(a_{i}, b_{i}\right)$ and $\left(a_{i+1}, b_{i+1}\right)$ by [M1, Proposition 0.9.1]. But if $\left\{a_{i}, b_{i}\right\} \cap\left\{a_{i+1}, b_{i+1}\right\}=\emptyset$, then we cannot permute $\left\{a_{i}, b_{i}\right\}$ and $\left\{a_{i+1}, b_{i+1}\right\}$ in general. From now on we assume that in a chain $\mathfrak{p}$,

if $\left\{a_{i}, b_{i}\right\} \cap\left\{a_{j}, b_{j}\right\}=\emptyset$ for $i<j$, it does not happen that $a_{i}>a_{j}>b_{j}>b_{i}$, in order to use [M1, Cor. 0.10.2] on non-vanishing of normalized intertwining operators. We note that the condition (3.1) is just a rephrasing of the condition (2) in Remark 3.2.

Remark 3.3. Let $O$ be a unipotent orbit obtained by ignoring the ordering in a chain $\mathfrak{p}=\left(a_{1}, b_{1}, \ldots, a_{s}, b_{s}, a_{s+1}\right)$. Suppose either $O$ is not distinguished or $\mathfrak{p}$ does not satisfy condition (1) in Remark 3.2. Then $\mathfrak{p}$ can be written as $\mathfrak{p}=\mathfrak{p}_{1} \times \cdots \mathfrak{p}_{k} \times \mathfrak{p}_{0}$, where $\mathfrak{p}_{i} \in P\left(O_{i}\right)$ and $O_{i}$ is a distinguished unipotent orbit in $G_{i}^{*}$ for $i=0,1, \ldots, k$. It means that it comes from global consideration when $\chi=\chi(\underbrace{\mu_{1}, \ldots, \mu_{1}}_{r_{1}}, \ldots, \underbrace{\mu_{k}, \ldots, \mu_{k}}_{r_{k}}, \underbrace{1, \ldots, 1}_{r_{0}})$, where $\mu_{i}, i=1, \ldots, k$ are non-trivial quadratic grössencharacters such that $\mu_{i v}=1$ for $i=1, \ldots, k$ for a given non-archimedean place $v$. 
For a segment $(a, b)$, we define the Weyl group elements $w_{(a, b)}, \sigma_{(a, b)}$ as follows (see [M1, p. 660]):

$$
\begin{aligned}
w_{(a, b)}(t) & =-t, \quad \text { if } 1 \leq t \leq \frac{a-b}{2}, \\
w_{(a, b)}\left(t+\frac{a-b}{2}\right) & =\frac{a+b}{2}+1-t, \quad \text { if } 1 \leq t \leq b, \\
\sigma_{(a, b)}(t) & =t, \quad \text { if } 1 \leq t \leq \frac{a-b}{2}, \\
\sigma_{(a, b)}\left(t+\frac{a-b}{2}\right) & =-\left(\frac{a+b}{2}+1-t\right), \quad \text { if } 1 \leq t \leq b .
\end{aligned}
$$

We note that

(1) $\sigma_{(a, b)} w_{(a, b)}=w_{(a, b)} \sigma_{(a, b)}$ is the longest element $w_{0}$ of the Weyl group of $S p_{2 n}, n=\frac{a+b}{2}$, i.e., $w_{0}=c_{1} c_{2} \cdots c_{n}$, where $c_{i}$ 's are sign changes.

(2) $\sigma_{(a, b)} \lambda_{(a, b)}=\lambda_{(a, b)}$.

(3) $l\left(\sigma_{(a, b)} w_{(a, b)}\right)=l\left(w_{(a, b)}\right)+l\left(\sigma_{(a, b)}\right)$.

(4) If $q=1, \sigma_{(a, 1)}=c_{n}$ and $w_{(a, 1)}=c_{1} \cdots c_{n-1}$.

For a segment attached to $(a)$, we define $w_{(a)}=c_{1} c_{2} \cdots c_{\frac{a-1}{2}}$ and $\sigma_{(a)}=1$. $\lambda_{(a)}$ is the intersection of the $\frac{a-1}{2}$ singular hyperplanes $e_{1}-e_{2}=1, e_{2}-e_{3}=1$, $\ldots, e_{\frac{a-3}{2}}-e_{\frac{a-1}{2}}=1$.

For a chain $\mathfrak{p}=\left(a_{1}, b_{1}, \ldots, a_{s}, b_{s}, a_{s+1}\right)$, we define $w_{\mathfrak{p}}$ as $w_{\mathfrak{p}}=$ $w_{\left(a_{1}, b_{1}\right)} \cdots w_{\left(a_{s}, b_{s}\right)} w_{\left(a_{s+1}\right)}$ with an obvious meaning and $\operatorname{Stab}\left(\lambda_{\mathfrak{p}}, \uparrow \mathfrak{p}\right)$ as the group generated by $\sigma_{\left(a_{i}, b_{i}\right)}$ for $i=1, \ldots, s$.

In order to apply induction, we define $\mathfrak{p}_{\geq i}=\left(a_{i}, b_{i}, \ldots, a_{s}, b_{s}, a_{s+1}\right)$. Let, for $1 \leq i \leq s, T_{i}^{d}=\sum_{k=1}^{i-1} \frac{a_{k}+b_{k}}{2}$ and $T_{i}^{f}=\sum_{k=1}^{i} \frac{a_{k}+b_{k}}{2}$.

Definition 3.2. For a chain $\mathfrak{p}=\left(a_{1}, b_{1}, \ldots, a_{s}, b_{s}, a_{s+1}\right)$, we define a Levi subgroup $M_{\mathfrak{p}}^{\prime}=G L\left(\frac{a_{1}+b_{1}}{2}\right) \times \cdots \times G L\left(\frac{a_{s}+b_{s}}{2}\right) \times G L\left(\left[\frac{a_{s+1}}{2}\right]\right)$ and degenerate principal series

$$
\begin{aligned}
\bar{I}\left(\lambda_{\mathfrak{p}}, \chi\right) & =\operatorname{Ind}_{M_{\mathfrak{p}}^{\prime}} \bar{\chi} \otimes|\operatorname{det}|^{\frac{a_{1}-b_{1}}{4}} \times \cdots \times|\operatorname{det}|^{\frac{a_{s}-b_{s}}{4}} \times|\operatorname{det}|^{\frac{1}{2}\left[\frac{a_{s+1}+1}{2}\right]}, \\
\bar{I}\left(-\lambda_{\mathfrak{p}}, \chi\right) & =\operatorname{Ind}_{M_{\mathfrak{p}}^{\prime}} \bar{\chi} \otimes|\operatorname{det}|^{-\frac{a_{1}-b_{1}}{4}} \times \cdots \times|\operatorname{det}|^{-\frac{a_{s}-b_{s}}{4}} \times|\operatorname{det}|^{-\frac{1}{2}\left[\frac{a_{s+1}+1}{2}\right]},
\end{aligned}
$$

where $\bar{\chi}$ is the character of $M_{\mathfrak{p}}^{\prime}$ induced by $\chi$.

If we set $w_{\mathfrak{p}}^{\prime}$ to be the longest Weyl group element of $M_{\mathfrak{p}}^{\prime}$, then $\bar{I}\left(\lambda_{\mathfrak{p}}, \chi\right)$ is the image of the normalized intertwining operator $R\left(w_{\mathfrak{p}}^{\prime}, \lambda_{\mathfrak{p}}, \chi\right)$.

The normalized intertwining operator $R\left(w_{\mathfrak{p}}, \lambda, \chi\right)$ is not holomorphic at $\lambda_{\mathfrak{p}}$. In order to define $R\left(w_{\mathfrak{p}}, \lambda_{\mathfrak{p}}, \chi\right)$, we need:

Proposition 3.1. For each segment $\left(a_{i}, b_{i}\right), R\left(w_{\left(a_{i}, b_{i}\right)}, \lambda_{\left(a_{1}, b_{1}\right)} \cdots \lambda_{\left(a_{i}, b_{i}\right)}, \chi\right)$ defines a holomorphic intertwining operator from $\operatorname{Ind}_{G L(1) \times \cdots \times G L(1) \times G\left(n-T_{i}^{f}\right)} \chi_{\left(a_{1}, b_{1}\right)} \lambda_{\left(a_{1}, b_{1}\right)} \cdots \chi_{\left(a_{i}, b_{i}\right)} \lambda_{\left(a_{i}, b_{i}\right)} \times \bar{I}\left(-\lambda_{\mathfrak{p}_{\geq i+1}}, \chi\right)$, 
into

$$
\begin{aligned}
\operatorname{Ind}_{G L(1) \times \cdots \times G L(1) \times G\left(n-T_{i}^{f}\right)} \chi_{\left(a_{1}, b_{1}\right)} & \lambda_{\left(a_{1}, b_{1}\right)} \cdots \chi_{\left(a_{i-1}, b_{i-1}\right)} \lambda_{\left(a_{i-1}, b_{i-1}\right)} \\
& \times w_{\left(a_{i}, b_{i}\right)} \chi_{\left(a_{i}, b_{i}\right)} \lambda_{\left(a_{i}, b_{i}\right)} \times \bar{I}\left(-\lambda_{\mathfrak{p}_{\geq i+1}}, \chi\right) .
\end{aligned}
$$

Its image is included in

$$
\begin{aligned}
& \operatorname{Ind}_{G L(1) \times \cdots \times G L(1) \times G\left(n-T_{i+1}^{f}\right)} \chi_{\left(a_{1}, b_{1}\right)} \lambda_{\left(a_{1}, b_{1}\right)} \cdots \\
& \quad \chi_{\left(a_{i-1}, b_{i-1}\right)} \lambda_{\left(a_{i-1}, b_{i-1}\right)} \times \bar{I}\left(-\lambda_{\mathfrak{p}_{\geq i}}, \chi\right) .
\end{aligned}
$$

Proof. The argument is like that in [M1, 0.13]; the introduction of quadratic characters does not create any new complications.

We define the normalized intertwining operator $R\left(w_{\mathfrak{p}}, \lambda_{\mathfrak{p}}, \chi\right)$ as the composition of the above operators. Then

$$
\begin{aligned}
R\left(w_{\mathfrak{p}}, \lambda_{\mathfrak{p}}, \chi\right) \subset \bar{I}\left(-\lambda_{\mathfrak{p}}, \chi\right) & \\
R\left(w_{\mathfrak{p}}, \lambda_{\mathfrak{p}}, \chi\right) \subset \operatorname{Ind}_{G L\left(\frac{a_{1}+b_{1}}{2}\right) \times G\left(n-T_{1}^{f}\right)} \mu_{1}|\operatorname{det}|^{-\frac{a_{1}-b_{1}}{4}} & \quad \times R\left(w_{\mathfrak{p}_{\geq 2}}, \lambda_{\mathfrak{p} \geq 2}, \chi\right) I\left(\lambda_{\mathfrak{p}_{\geq 2}}, \chi\right) .
\end{aligned}
$$

Here $\chi$ in $R\left(w_{\mathfrak{p}_{\geq 2}}, \lambda_{\mathfrak{p}_{\geq 2}}, \chi\right) I\left(\lambda_{\mathfrak{p}_{\geq 2}}, \chi\right)$ should be interpreted appropriately.

Lemma 3.2. The normalized intertwining operator $R\left(w_{\mathfrak{p}}, \lambda_{\mathfrak{p}}, \chi\right)$ does not vanish identically.

Proof. Let $\lambda_{O}$ be the conjugate of $\lambda_{\mathfrak{p}}$ which is in the closure of the positive Weyl chamber. Let $w_{1}$ be a Weyl group element such that $\lambda_{\mathfrak{p}}=w_{1} \lambda_{O}$. Consider the following commutative diagram.

$$
\begin{aligned}
& I\left(\lambda_{O}, w_{1}^{-1} \chi\right) \stackrel{R\left(w_{O}, \lambda_{O}, w_{1}^{-1} \chi\right)}{\longrightarrow} I\left(-\lambda_{O}, w_{1}^{-1} \chi\right) \\
& \downarrow R\left(w_{1}, \lambda_{O}, w_{1}^{-1} \chi\right) \quad \uparrow R\left(w_{1}^{-1},-\lambda_{\mathfrak{p}}, \chi\right) \\
& I\left(\lambda_{\mathfrak{p}}, \chi\right) \stackrel{R\left(w_{\mathfrak{p}}, \lambda_{\mathfrak{p}}, \chi\right)}{\longrightarrow} \quad I\left(-\lambda_{\mathfrak{p}}, \chi\right) .
\end{aligned}
$$

Here $R\left(w_{O}, \lambda_{O}, w_{1}^{-1} \chi\right)$ is the intertwining operator on $\operatorname{Ind}_{P}^{G} \lambda_{O} \otimes \operatorname{Ind}_{B}^{M} w_{1}^{-1} \chi$, where $P=M N$ is the parabolic subgroup such that $\lambda_{O}$ is in the positive Weyl chamber with respect to $P$. Then it is non-vanishing. Note that all the normalized intertwining operators are holomorphic. Therefore, $R\left(w_{\mathfrak{p}}, \lambda_{\mathfrak{p}}, \chi\right)$ is non-vanishing.

We first reduce to the case $\chi=1$. 
3.1. Review of the results of Barbasch-Moy [B-Mo2]. We now consider the case when $\chi$ is unramified. The following discussion is based on [B-Mo2].

Let $G=S O(2 n+1), \theta=||{ }^{a_{1}} \mu \times \cdots \times||^{a_{n_{1}}} \mu \times||^{b_{1}} \times \cdots \times||^{b_{n_{0}}}$, a character of $T \subset G\left(n_{0}+n_{1}=n\right)$, where $\mu$ is the non-trivial unramified quadratic character and $a_{1}, \ldots, a_{n_{1}}, b_{1}, \ldots, b_{n_{0}} \in \mathbb{R}$. Let $H^{\prime}=H_{1}^{\prime} \times H_{0}^{\prime}$, with $H_{1}^{\prime}=$ $S O\left(2 n_{1}+1\right), H_{0}^{\prime}=S O\left(2 n_{0}+1\right)$. Set $\theta^{\prime}=\theta_{1}^{\prime} \times \theta_{0}^{\prime}$ with $\theta_{1}^{\prime}=||^{a_{1}} \times \cdots \times||^{a_{n_{1}}}$, $\theta_{0}^{\prime}=||^{b_{1}} \times \cdots \times||^{b_{n_{0}}}$ characters of $T_{1}^{\prime} \subset H_{1}^{\prime}, T_{0}^{\prime} \subset H_{0}^{\prime}$. If $\tau$ (resp. $\tau^{\prime}, \tau_{1}^{\prime}$, $\left.\tau_{0}^{\prime}\right)$ denotes the infinitesimal character associated to subquotients of $\operatorname{Ind}_{B}^{G} \theta$ (resp. $\operatorname{Ind}_{B^{\prime}}^{H^{\prime}} \theta^{\prime}, \operatorname{Ind}_{B_{1}^{\prime}}^{H_{1}^{\prime}} \theta_{1}^{\prime}, \operatorname{Ind}_{B_{0}^{\prime}}^{H_{0}^{\prime}} \theta_{0}^{\prime}$ ), then the results of [B-Mo2] tell us that there is an equivalence of categories

$$
\mathcal{R}(G, \tau) \simeq \mathcal{R}\left(H^{\prime}, \tau^{\prime}\right) \simeq \mathcal{R}\left(H_{1}^{\prime} \times H_{0}^{\prime}, \tau_{1}^{\prime} \times \tau_{0}^{\prime}\right),
$$

where $\mathcal{R}(G, \tau)$ denotes the category of smooth finite-length representations of $G$ with infinitesimal character $\tau$.

Suppose $G=S p(2 n)$. Then the same discussion as above applies, except that in this case $H_{1}^{\prime}=O\left(2 n_{1}\right)$ and $H_{0}^{\prime}=S p\left(2 n_{0}\right)$.

Ultimately, we are going to apply the results of [B-Mo2] to those of $[\mathbf{R}]$ to deal with representations of $S p(2 n)$ and $S O(2 n+1)$. Therefore, we also need to discuss [B-Mo2] for $O(2 n)$ (cf. Example 1.1). Unfortunately, the results of [B-Mo2] do not apply to disconnected groups, nor does there appear to be any obvious way to extend the results of [B-Mo2] from $S O(2 n)$ to $O(2 n)$. Thus, we are forced to assume the results of [B-Mo2] hold for $O(2 n)$ as well (cf. Assumption 3.1.1 below). The above discussion then applies to $G=O(2 n)$ as well. In this case, $H_{1}^{\prime}=O\left(2 n_{1}\right)$ and $H_{0}^{\prime}=O\left(2 n_{0}\right)$.

Assumption 3.1.1. The results in Sections 1-6 of [B-Mo2] hold for $O(2 n)$.

Next, we note that this equivalence respects induction, in a suitable sense. Let $G$ be one of the groups above $(S O(2 n+1), S p(2 n), O(2 n))$ and $M$ a standard Levi subgroup of $G$. Let $M^{\prime}, H^{\prime}$ be the groups corresponding to $M, G$ under [B-Mo2]. Let $\tau_{M}, \tau_{M^{\prime}}^{\prime}$ be infinitesimal characters for $M, M^{\prime}$ which correspond under [B-Mo2], $\tau, \tau^{\prime}$ the infinitesimal characters for $G, H^{\prime}$ obtained by induction. Then by [B-Mo2, Theorem 6.2], the following diagram commutes:

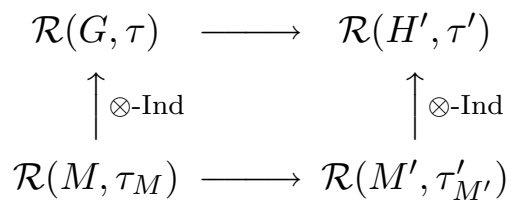

where $\otimes$-Ind denotes induction defined via tensor product at the Hecke algebra level.

Example 3.1.1. Let $\alpha_{1}, \ldots, \alpha_{k} \in \mathbb{R}$ and

$$
\pi=\operatorname{Ind}_{P}^{G}\left(\left|\operatorname{det}_{m_{1}}\right|^{\alpha_{1}} \mu \circ \operatorname{det}_{m_{1}} \times \cdots\right.
$$




$$
\begin{gathered}
\left.\times\left|\operatorname{det}_{m_{j}}\right|^{\alpha_{j}} \mu \circ \operatorname{det}_{m_{j}} \times\left|\operatorname{det}_{m_{j+1}}\right|^{\alpha_{j+1}} \times \cdots \times\left|\operatorname{det}_{m_{k}}\right|^{\alpha_{k}}\right) \\
\pi^{\prime}=\operatorname{Ind}_{P^{\prime}}^{H^{\prime}}\left(\left|\operatorname{det}_{m_{1}}\right|^{\alpha_{1}} \times \cdots \times\left|\operatorname{det}_{m_{j}}\right|^{\alpha_{j}} \times\left|\operatorname{det}_{m_{j+1}}\right|^{\alpha_{j+1}} \times \cdots \times\left|\operatorname{det}_{m_{k}}\right|^{\alpha_{k}}\right),
\end{gathered}
$$

where $P, P^{\prime}$ are standard parabolics of $G, H^{\prime}$ whose Levi factors both have the form $G L\left(m_{1}\right) \times \cdots \times G L\left(m_{k}\right)$. Observe that by [Ja1, Proposition 2.1.2] (which may be extended to cover $O(2 n)$; we may choose $w_{0}=c_{1} c_{2} \cdots c_{n}$, and similarly for smaller rank orthogonal groups appearing in standard Levi subgroups), we have

$$
\begin{aligned}
\pi \cong \otimes & -\operatorname{Ind}_{P}^{G}\left(\left|\operatorname{det}_{m_{1}}\right|^{-\alpha_{1}} \mu \circ \operatorname{det}_{m_{1}} \times \cdots\right. \\
& \left.\times\left|\operatorname{det}_{m_{j}}\right|^{-\alpha_{j}} \mu \circ \operatorname{det}_{m_{j}} \times\left|\operatorname{det}_{m_{j+1}}\right|^{-\alpha_{j+1}} \times \cdots \times\left|\operatorname{det}_{m_{k}}\right|^{-\alpha_{k}}\right) \\
\pi^{\prime} \cong \otimes & -\operatorname{Ind}_{P^{\prime}}^{H^{\prime}}\left(\left|\operatorname{det}_{m_{1}}\right|^{-\alpha_{1}} \times \cdots\right. \\
& \left.\times\left|\operatorname{det}_{m_{j}}\right|^{-\alpha_{j}} \times\left|\operatorname{det}_{m_{j+1}}\right|^{-\alpha_{j+1}} \times \cdots \times\left|\operatorname{det}_{m_{k}}\right|^{-\alpha_{k}}\right) .
\end{aligned}
$$

Since the inducing representation for $\pi$ is the unique irreducible subrepresentation of

$$
\begin{aligned}
\operatorname{Ind}_{B_{M}}^{M} & \left(\left(||^{\alpha_{1}+\frac{-m_{1}+1}{2}} \mu \times \cdots \times||^{\alpha_{1}+\frac{m_{1}-1}{2}} \mu\right) \times \cdots\right. \\
& \times\left(||^{\alpha_{j}+\frac{-m_{j}+1}{2}} \mu \times \cdots \times||^{\alpha_{j}+\frac{m_{j}-1}{2}} \mu\right) \\
& \times\left(||^{\alpha_{j+1}+\frac{-m_{j+1}+1}{2}} \times \cdots \times||^{\alpha_{j+1}+\frac{m_{j+1}-1}{2}}\right) \times \cdots \\
& \left.\times\left(||^{\alpha_{k}+\frac{-m_{k}+1}{2}} \times \cdots \times||^{\alpha_{k}+\frac{m_{k}-1}{2}}\right)\right),
\end{aligned}
$$

and similarly for $\pi^{\prime}$, we see that the inducing representations correspond under $\mathcal{R}\left(M, \tau_{M}\right) \simeq \mathcal{R}\left(M^{\prime}, \tau_{M^{\prime}}^{\prime}\right)$. By the discussion above, this means that $\pi$ and $\pi^{\prime}$ correspond under $\mathcal{R}(G, \tau) \simeq \mathcal{R}\left(H^{\prime}, \tau^{\prime}\right)$.

3.2. Matching of images of intertwining operators under Hecke algebra isomorphisms. Let $\chi=\chi(\underbrace{\mu_{1}, \ldots, \mu_{1}}_{r_{1}}, \ldots, \underbrace{\mu_{k}, \ldots, \mu_{k}}_{r_{k}}, \underbrace{1, \ldots, 1}_{r_{0}})$, where $r_{0}+\cdots+r_{k}=n, r_{1} \geq \cdots \geq r_{k}$, and the $\mu_{i}$ 's are distinct local quadratic characters. Let $G^{\prime}=G_{1}^{\prime} \times \cdots \times G_{k}^{\prime} \times G_{0}^{\prime}$. Combining the Hecke algebra isomorphisms of Roche and the graded algebra isomorphisms of Barbasch-Moy, we get an equivalence of categories, which gives rise to a correspondence between subquotients of the induced representation $I^{G}(\lambda, \chi)$ and subquotients of the induced representation $I^{G^{\prime}}(\lambda, 1)$ (cf. Example 1.1 and previous section). Note that if we write $\lambda=\lambda_{1} \times \cdots \times \lambda_{k} \times \lambda_{0}$ with $\lambda_{1}=||^{a_{1}} \times \cdots \times||^{a_{r}}, a_{1}, \ldots, a_{r} \in \mathbb{R}$, etc. we have $I^{G^{\prime}}(\lambda, 1) \cong$ $I^{G_{1}^{\prime}}\left(\lambda_{1}, 1\right) \times \cdots \times I^{G_{k}^{\prime}}\left(\lambda_{k}, 1\right) \times I^{G_{0}^{\prime}}\left(\lambda_{0}, 1\right)$. The correspondence preserves temperedness, square-integrability, etc. The correspondence behaves well with respect to intertwining operators: 
Proposition 3.2.1. The above correspondence behaves well with respect to intertwining operators, i.e., $R\left(w_{\mathfrak{p}}, \lambda_{\mathfrak{p}}, \chi\right) I\left(\lambda_{\mathfrak{p}}, \chi\right)$ corresponds to

$$
R\left(w_{\mathfrak{p}_{1}}, \lambda_{\mathfrak{p}_{1}}\right) I^{G_{1}^{\prime}}\left(\lambda_{\mathfrak{p}_{1}}\right) \times \cdots \times R\left(w_{\mathfrak{p}_{k}}, \lambda_{\mathfrak{p}_{k}}\right) I^{G_{k}^{\prime}}\left(\lambda_{\mathfrak{p}_{k}}\right) \times R\left(w_{\mathfrak{p}_{0}}, \lambda_{\mathfrak{p}_{0}}\right) I^{G_{0}^{\prime}}\left(\lambda_{\mathfrak{p}_{0}}\right),
$$

where $w_{\mathfrak{p}}=w_{\mathfrak{p}_{1}} \times \cdots \times w_{\mathfrak{p}_{k}} \times w_{\mathfrak{p}_{0}}$ and $\lambda_{\mathfrak{p}}=\lambda_{\mathfrak{p}_{1}} \times \cdots \times \lambda_{\mathfrak{p}_{k}} \times \lambda_{\mathfrak{p}_{0}}$.

We prove this in this section. The arguments used in this section are based largely on $[\mathbf{C a}],[\mathbf{C a} 2],[\mathbf{R e} 2]$ (with their presentation also influenced by $[\operatorname{Re} 1])$.

Since we are assuming $F$ has odd residual characteristic here, we have that $F$ admits three non-trivial quadratic characters. Let $\mu$, resp. $\mu_{n r}$, denote a ramified, resp. unramified, non-trivial quadratic character (so that $\mu, \mu_{n r}, \mu \mu_{n r}$ are the three non-trivial quadratic characters). If we fix a uniformizer $\varpi$, we may assume $\mu$ is the ramified quadratic character satisfying $\mu(\varpi)=1$. For convenience, assume $\chi$ (cf. Section 1) has the form

$$
\chi=\underbrace{\mu \times \cdots \times \mu}_{n_{1}} \times \underbrace{1 \times \cdots \times 1}_{n_{0}} .
$$

(As in Example 1.1, it is actually $\left.\chi\right|_{T(\mathcal{O})}$ that is needed in Roche's construction.) Then we have $J_{\chi}=I=$ Iwahori subgroup and

$$
\mathcal{H}(G, \rho) \simeq \mathcal{H}\left(\tilde{H}, 1_{I_{H}}\right) .
$$

Here $\rho=\rho_{\chi}$ as in Section 1. It will be convenient to write $H^{\prime}$ (resp., $\left.I^{\prime}, B^{\prime}\right)$ for $\tilde{H}$ (resp., $\left.I_{H}, B_{H}\right)$. Recall the decomposition $H^{\prime}=H_{1}^{\prime} \times H_{0}^{\prime}$ from Example 1.1.

Now, $\mathcal{H}(G, \rho)$ has linear basis $\left\{T_{w}\right\}_{w \in W_{\chi}}$, where $T_{w}$ is supported on $I w I$. If we identify $W_{\chi}$ with representatives in $G$ chosen as in the proof of Lemma $9.3[\mathbf{R}]$ (which in turn is based on $[\mathbf{M r}]$ ), we can normalize $T_{w}$ so that it is 1 at $w$. Also, observe that $\bar{W}_{\chi}=\bar{W}^{\prime}$, which may be identified with $\bar{W}\left(H_{1}^{\prime}\right) \times \bar{W}\left(H_{0}^{\prime}\right)$. We let $w_{0}$ denote the longest element of $\bar{W}$, and note that $w_{0} \in \bar{W}_{\chi}$.

If $\pi=\operatorname{Ind}_{B}^{G}(\lambda \chi)$ with $\lambda$ unramified, then $V_{\pi}^{I, \chi}$ has as basis $\left\{f_{w}\right\}_{w \in \bar{W}_{\chi}}$, where

$$
f_{w}(g)= \begin{cases}\delta^{\frac{1}{2}}(t) \lambda(t) \chi(t) \chi(i), & \text { if } g=(t u) w i \in B w I \\ 0, & \text { if not. }\end{cases}
$$

Similarly, if $\pi^{\prime}=\operatorname{Ind}_{B^{\prime}}^{H^{\prime}} \lambda$, then $V_{\pi^{\prime}}^{I^{\prime}}$ has basis $\left\{f_{w}^{\prime}\right\}_{w \in \overline{W^{\prime}}}$, where

$$
f_{w}^{\prime}\left(g^{\prime}\right)= \begin{cases}\delta^{\prime \frac{1}{2}}\left(t^{\prime}\right) \lambda\left(t^{\prime}\right), & \text { if } g^{\prime}=\left(t^{\prime} u^{\prime}\right) w i^{\prime} \in B^{\prime} w I^{\prime} \\ 0, & \text { if not. }\end{cases}
$$

If we let $T_{w}^{\prime}, w \in W^{\prime}$, denote the characteristic function of $I^{\prime} w I^{\prime}$, we have the following: 
Lemma 3.2.2. Let $s \in \overline{W^{\prime}}$ be the reflection associated to the $\chi$-simple root $\alpha \in \Pi_{\chi}$. For $w \in \overline{W^{\prime}}$, we have

$$
\pi^{\prime}\left(T_{s}^{\prime}\right) f_{w}^{\prime}= \begin{cases}f_{w s}^{\prime}, & \text { if } w \alpha>0, \\ q f_{w s}^{\prime}+(q-1) f_{w}^{\prime}, & \text { if } w \alpha<0 .\end{cases}
$$

Further, for $c \in C_{\chi}$,

$$
\pi^{\prime}\left(T_{c}\right) f_{w}^{\prime}=f_{w c^{-1}}^{\prime}
$$

Proof. Since it is well-known how to do such calculations (and straightforward), we omit the details.

Corollary 3.2.3. Let $\mathcal{H}(K, \rho) \subset \mathcal{H}(G, \rho)$ denote the subalgebra consisting of functions supported on $K$. Then, $f_{w_{0}}$ generates $V_{\pi}^{I, \chi}$ under the action of $\mathcal{H}(K, \rho)$.

Proof. Observe that the corresponding result for $\pi^{\prime}$ is straightforward: From the preceding lemma, $f_{w}^{\prime}=\pi^{\prime}\left(T_{w^{-1}}^{\prime}\right) f_{1}^{\prime}$. Therefore, $f_{1}^{\prime}=\pi^{\prime}\left(T_{w_{0}^{-1}}^{\prime}{ }^{-1}\right) f_{w_{0}}^{\prime}$. Thus, $f_{w}^{\prime}=\pi^{\prime}\left(T_{w^{-1}}^{\prime}\right) \pi^{\prime}\left(T_{w_{0}^{-1}}^{\prime}{ }^{-1}\right) f_{w_{0}}^{\prime}$. To extend this to cover $\pi$, observe that $\left.f_{w}^{\prime}\right|_{K^{\prime}}=T_{w}^{\prime}$ and $\left.f_{w}\right|_{K}=T_{w}$. Therefore, for $w_{1}, w_{2} \in \bar{W}_{\chi}$, if $T_{w_{2}}^{\prime} *$ $T_{w_{1}^{-1}}^{\prime}=\sum c_{w} T_{w}^{\prime}$, then $\pi^{\prime}\left(T_{w_{1}}^{\prime}\right) f_{w_{2}}^{\prime}=\sum c_{w} f_{w}^{\prime}$, and similarly for $\pi$. Suppose $\Psi: T_{w} \mapsto a_{w} T_{w}^{\prime}$. If $T_{w_{1}^{-1}}^{\prime} * T_{w_{2}}^{\prime}=\sum c_{w} T_{w}^{\prime}$, one can then conclude that $\pi\left(T_{w_{1}}\right) f_{w_{2}}=\sum c_{w} a_{w_{1}} a_{w_{2}} a_{w}^{-1} f_{w}$. The corollary follows.

If $B=T U$ is the Levi factorization of $B$, we use $\pi_{U}$ to denote the (unnormalized) Jacquet module of $\pi$ with respect to $U$. It has

space: $\left(V_{\pi}\right)_{U}=V_{\pi} / V_{\pi}(U)$,

$$
\text { where } V_{\pi}(U)=\operatorname{span}\left\{\pi(u) v-v \mid u \in U, v \in V_{\pi}\right\} \text {, }
$$

action: $\pi_{U}(t)\left(v+V_{\pi}(U)\right)=\pi(t) v+V_{\pi}(U)$.

We have the following:

Lemma 3.2.4. The restriction of the quotient map $V_{\pi} \longrightarrow\left(V_{\pi}\right)_{U}$ to $V_{\pi}^{I, \rho}$ gives rise to an isomorphism $V_{\pi}^{I, \rho} \simeq\left(V_{\pi}\right)_{U}^{T_{0}, \chi}$ as vector spaces.

Proof. The proof is essentially the same as that done by Casselman for unramified principal series, so we just give a sketch here.

First, one checks that if $v \in V_{\pi}^{U_{1}^{-} T_{0}, \chi}$, then $v-\pi\left(e_{\rho}\right) v \in V_{\pi}(U)$ (note that $\left.e_{\rho}=T_{1}\right)$. Consequently, $\left(V_{\pi}^{I, \rho}\right)_{U}=\left(V_{\pi}^{U_{1}^{-} T_{0}, \chi}\right)_{U}$. Next, we choose a finitedimensional subspace $X \subset V_{\pi}^{T_{0}, \chi}$ which maps onto $\left(V_{\pi}^{T_{0}, \chi}\right)_{U}$. If we take a compact subgroup $U_{k}^{-} \subset U^{-}$which acts trivially on $X$ and $t \in T$ such that $t^{-1} U_{1}^{-} t \subset U_{k}^{-}$, we get $\pi(t) X \subset V_{\pi}^{T_{0} U_{1}^{-}, \chi}$. Therefore, $\left(V_{\pi}^{T_{0}, \chi}\right)_{U}=\pi_{U}(t) X_{U} \subset$ $\left(V_{\pi}^{T_{0} U_{1}^{-}, \chi}\right)_{U}=\left(V_{\pi}^{I, \rho}\right)_{U}$, giving surjectivity. Injectivity then follows from a comparison of dimensions. 
Lemma 3.2.5. For $t \in T^{-}, \pi\left(T_{t}\right) f_{w_{0}}=\delta^{-\frac{1}{2}}(t)\left(w_{0} \lambda\right)(t)\left(w_{0} \chi\right)(t) f_{w_{0}}$. (This result also holds when $G=O(2 n)$ and $w_{0}=c_{1} c_{2} \cdots c_{n}$.)

Proof. Recall that we have a $B$-stable filtration $V_{\pi}=\bigcup_{w \in \bar{W}}\left(V_{\pi}\right)_{w}$, where

$$
\left(V_{\pi}\right)_{w}=\left\{f \in V_{\pi} \mid \operatorname{supp} f \subset \bigcup_{x \geq w} B x B\right\},
$$

(where $\geq$ denotes the Bruhat order). This gives rise to a $T$-filtration $\left(V_{\pi}\right)_{U}=$ $\bigcup_{w \in \bar{W}}\left(\left(V_{\pi}\right)_{w}\right)_{U}$. By Lemma 6.3.5 [Ca] (also, see the proof of Lemma 2.12 $[\mathbf{B}-\mathbf{Z}]), T$ acts on $\left(\left(V_{\pi}\right)_{w_{0}}\right)_{U}$ by the character $\delta^{\frac{1}{2}} w_{0}(\lambda \chi)$. Since $B w_{0} I \subset$ $B w_{0} B$, we see that $f_{w_{0}}$ is a basis for $V_{\pi}^{I, \rho} \cap\left(V_{\pi}\right)_{w_{0}}$. Thus, by Lemma 3.2.4, $f_{w_{0}}+V_{\pi}(U)$ is a basis for $\left(\left(V_{\pi}\right)_{w_{0}}\right)_{U}$. In particular,

$$
\pi(t)\left(f_{w_{0}}+V_{\pi}(U)\right)=\delta^{\frac{1}{2}}(t)\left(w_{0} \lambda\right)(t)\left(w_{0} \chi\right)(t) f_{w_{0}}+V_{\pi}(U) .
$$

Next, the same basic argument used in [Ca2, Proposition 2.5] tells us that $|I t I|^{-1} \pi\left(T_{t}\right) f_{w_{0}}$ and $\pi(t) f_{w_{0}}$ have the same image in $\left(V_{\pi}\right)_{U}$. Since $|I t I|=$ $\delta^{-1}(t)([\mathbf{C a}$, Lemma 1.5.1]), we see that

$$
\begin{aligned}
\pi\left(T_{t}\right) f_{w_{0}}+V_{\pi}(U) & =\delta^{-1}(t) \pi(t) f_{w_{0}}+V_{\pi}(U) \\
& =\delta^{-\frac{1}{2}}(t)\left(w_{0} \lambda\right)(t)\left(w_{0} \chi\right)(t) f_{w_{0}}+V_{\pi}(U) .
\end{aligned}
$$

Finally, if one writes $\pi\left(T_{t}\right) f_{w_{0}}=\sum_{w \in \bar{W}_{\chi}} c_{w} f_{w}$, taking Jacquet modules gives $\pi\left(T_{t}\right) f_{w_{0}}+V_{\pi}(U)=\sum_{w \in \bar{W}_{\chi}} c_{w} f_{w}+V_{\pi}(U)$. It is now immediate from the preceding lemma and the equality above that $c_{w_{0}}=\delta^{-\frac{1}{2}}(t)\left(w_{0} \lambda\right)(t)\left(w_{0} \chi\right)(t) f_{w_{0}}$ and $c_{w}=0$ for $w \neq w_{0}$.

For $G=O(2 n)$, let $\lambda, \chi$ be as above and $w_{0}=c_{1} c_{2} \cdots c_{n}$. Let $\bar{G}=S O(2 n)$ and $\bar{w}_{0} \in \bar{W}(\bar{G})$ of maximal length. The preceding argument then tells us that for $\bar{\pi}=\operatorname{Ind}_{B}^{\bar{G}} \lambda \chi, \bar{\pi}\left(T_{t}\right) f_{\bar{w}_{0}}=\delta^{-\frac{1}{2}}(t)\left(\bar{w}_{0} \lambda\right)(t)\left(\bar{w}_{0} \chi\right)(t) f_{\bar{w}_{0}}$ when $t \in T^{-}$. If $\bar{w}_{0}=w_{0}(n$ even $)$, the $O(2 n)$ result is immediate. If $\bar{w}_{0}=w_{0} c_{n}(n$ odd $)$, it follows easily from the fact that $\pi\left(e_{c_{n}}\right) f_{\bar{w}_{0}}=f_{w_{0}}$ and $\delta \circ c_{n}=\delta$.

For $\lambda, \chi$ as above, let $\pi=\operatorname{Ind}_{B}^{G}(\lambda \chi)$ and $\pi^{\prime}=\operatorname{Ind}_{B^{\prime}}^{H^{\prime}}(\lambda)$. If $\lambda=||^{x_{1}} \times \cdots \times$ ||$^{x_{1}} \times||^{y_{1}} \times \cdots \times||^{y_{n_{0}}}$, we set $\lambda_{1}=||^{x_{1}} \times \cdots \times||^{x_{n_{1}}}$ and $\lambda_{0}=||^{y_{1}} \times \cdots \times||^{y_{n_{0}}}$ (so that $\lambda=\lambda_{1} \times \lambda_{0}$ ). We note that $\pi^{\prime} \simeq \pi_{1}^{\prime} \otimes \pi_{0}^{\prime}$, where $\pi_{1}^{\prime}=\operatorname{Ind}_{B_{1}^{\prime}}^{H_{1}^{\prime}} \lambda_{1}$ and $\pi_{0}^{\prime}=\operatorname{Ind}_{B_{0}^{\prime}}^{H_{0}^{\prime}} \lambda_{0}$. We define a map $\mathfrak{M}: V_{\pi}^{I, \chi} \longmapsto V_{\pi^{\prime}}^{I^{\prime}}$ as follows: Let $\mathfrak{M}: f_{w_{0}} \longmapsto f_{w_{0}}^{\prime}$. (Note that under the identification $\bar{W}_{\chi}=\bar{W}^{\prime}=\bar{W}\left(H_{1}^{\prime}\right) \times$ $\bar{W}\left(H_{0}^{\prime}\right), w_{0}$ corresponds to $w_{0,1} \cdot w_{0,0}$, with $w_{0, i} \in \bar{W}\left(H_{i}^{\prime}\right)$ consisting of all sign changes. Under the identification $\pi^{\prime} \simeq \pi_{1}^{\prime} \otimes \pi_{0}^{\prime}$, we have $\left.f_{w_{0}}^{\prime} \longmapsto f_{w_{0,1}}^{\prime} \otimes f_{w_{0,0}}^{\prime}.\right)$ Then by Corollary 3.2.3, we can extend $\mathfrak{M}$ to get a linear isomorphism satisfying $\pi^{\prime}(\Psi(h))(\mathfrak{M} f)=\mathfrak{M}(\pi(h) f)$ for all $h \in \mathcal{H}(K, \rho), f \in V_{\pi}^{I, \chi}$. We claim that the equivalence of categories $\mathcal{R}_{\chi}(G) \simeq \mathcal{R}_{1}\left(H^{\prime}\right)$ comes from the map $\mathfrak{M}$. More precisely, we have the following: 
Proposition 3.2.6. The pair $(\mathcal{H}(G, \rho), \pi)$ is equivalent to $\left(\mathcal{H}\left(H^{\prime}, 1\right), \pi^{\prime}\right)$ under $(\Psi, \mathfrak{M})$. In particular,

$$
\pi^{\prime}(\Psi(h))(\mathfrak{M} f)=\mathfrak{M}(\pi(h) f)
$$

for all $h \in \mathcal{H}(G, \rho), f \in V_{\pi}^{I, \chi}$.

Proof. By definition, $\pi^{\prime}(\Psi(h))(\mathfrak{M} f)=\mathfrak{M}(\pi(h) f)$ holds for $h \in \mathcal{H}(K, \rho)$. Take $y \in Y^{+}$. Then $\Psi: \delta^{\frac{1}{2}}(y) T_{y} \longmapsto \delta^{\prime \frac{1}{2}}(y) T_{y}^{\prime}$ (cf. proof of Lemma $9.3[\mathbf{R}]$ ). We note that by definition, $T_{y}=T_{y(\tilde{w})}$. Since the extension of $\chi$ from $T(\mathcal{O})$ to $T$ satisfies $\chi(y(\tilde{w}))=1$ for all $y \in Y$, it follows from Lemma 3.2.5 that $\pi^{\prime}\left(\Psi\left(T_{y}\right)\right)\left(\mathfrak{M} f_{w_{0}}\right)=\mathfrak{M}\left(\pi\left(T_{y}\right) f_{w_{0}}\right)$ for $y \in Y^{+}$. Therefore, $\pi^{\prime}\left(\Psi\left(T_{y}\right)\right)(\mathfrak{M} f)=$ $\mathfrak{M}\left(\pi\left(T_{y}\right) f\right)$ for all $y \in Y, f \in V_{\pi}^{I, \chi}$. The proposition follows.

We now give a technical lemma which we will need below. In the lemma, we use $l_{G}$ to denote length for $\bar{W}(G), l_{\tilde{H}}$ for length for $\bar{W}(\tilde{H})$.

Lemma 3.2.7. Let $G, \chi$ be as above, $M$ the Levi factor of a standard parabolic subgroup of $G$. Then, there exists a set $\bar{W}_{\chi}^{T M} \subset \bar{W}_{\chi}$ such that the following all hold:

(1) $\bar{W}_{\chi}^{T M}$ is a set of representatives for $\bar{W}_{\chi}(M) \backslash \bar{W}_{\chi}$.

(2) For $x \in \bar{W}_{\chi}(M), w \in \bar{W}_{\chi}^{T M}$, we have $l_{G}(x w)=l_{G}(x)+l_{G}(w)$.

(3) For $x \in \bar{W}_{\chi}(M), w \in \bar{W}_{\chi}^{T M}$, we have $l_{\tilde{H}}(x w)=l_{\tilde{H}}(x)+l_{\tilde{H}}(w)$.

Proof. For explicitness, let $G=S p(2 n)$. Write $\chi=\underbrace{\mu \times \cdots \times \mu}_{n_{1}} \times \underbrace{1 \times \cdots \times 1}_{n_{0}}$ and $M \simeq G L\left(m_{1}\right) \times \cdots \times G L\left(m_{l}\right) \times S p\left(2 m_{0}\right)$, with $n_{0}+n_{1}=m_{0}+m_{1}+$ $\cdots+m_{l}=n$. Observe that $\tilde{H}=H \rtimes C_{\chi}$ with $C_{\chi}=\left\{1, c_{n_{1}}\right\}$, where $c_{n_{1}}$ denotes the $n_{1}$ th sign change. Let $\tilde{L}$ be the subgroup of $\tilde{H}$ corresponding to $M$ (cf. Lemma 1.4). We define

$$
\begin{aligned}
& \bar{W}^{T L}(H)=\left\{w \in \bar{W}(H) \mid w^{-1} \alpha>0 \text { for all } \alpha \in \Phi_{L}^{+}\right\} \\
& \bar{W}^{T L}(\tilde{H})=\left\{w \in \bar{W}(\tilde{H}) \mid w^{-1} \alpha>0 \text { for all } \alpha \in \Phi_{L}^{+}\right\} .
\end{aligned}
$$

It is known that if $x \in \bar{W}(L), w \in \bar{W}^{T L}(H)$, then $l_{\tilde{H}}(x w)=l_{\tilde{H}}(x)+l_{\tilde{H}}(w)$; since $l_{\tilde{H}}\left(c_{n_{1}}\right)=0$, this result clearly extends to $w \in \bar{W}^{T L}(\tilde{H})$. We consider two cases.

Case 1: $n_{1} \leq m_{1}+\cdots+m_{l}$.

In this case, $\tilde{L}=L$ is connected. Set $\bar{W}_{\chi}^{T M}=\bar{W}^{T L}(\tilde{H})$. Since $\bar{W}_{\chi}(M)=$ $\bar{W}(L)$, property (3) is immediate. The first property follows easily from the fact that $\bar{W}^{T L}(H)$ is a set of representatives for $\bar{W}(L) \backslash \bar{W}(H)$ and $\bar{W}^{T L}(\tilde{H})=\bar{W}^{T L}(H) \cup \bar{W}^{T L}(H) c_{n_{1}}$. 
Finally, for the second property, one can directly check that there is a standard Levi $M^{\prime}$ of $G$ such that $\Phi_{M, \chi}^{+}=\Phi_{L}^{+}=\Phi_{M^{\prime}}^{+}\left(\right.$if $n_{0}=m_{1}+\cdots+m_{i}$ for some $i, M^{\prime}=M$; otherwise, $\left.M^{\prime}<M\right)$. Then $\bar{W}_{\chi}(M)=\bar{W}\left(M^{\prime}\right)$ and $\bar{W}_{\chi}^{T M}=\bar{W}^{T L}(\tilde{H}) \subset \bar{W}^{T M^{\prime}}$. The result follows.

Case 2: $n_{1}>m_{1}+\cdots+m_{l}$.

In this case, $\tilde{L}=L \rtimes C_{\chi}$ is disconnected. Set $\bar{W}_{\chi}^{T M}=\bar{W}^{T M} \cap \bar{W}_{\chi}=$ $\left\{w \in \bar{W}_{\chi} \mid w^{-1} \alpha>0\right.$ for all $\left.\alpha \in \Phi_{M}^{+}\right\}$. As a first step, we check that $\bar{W}^{T L}(\tilde{H})=\bar{W}_{\chi}^{T M} \cup c_{n_{1}} \bar{W}_{\chi}^{T M}$. It follows easily from the definitions that $\bar{W}_{\chi}^{T M} \cup c_{n_{1}} \bar{W}_{\chi}^{T M} \subset \bar{W}^{T L}(\tilde{H})$. For the reverse containment, observe that $w \in \bar{W}^{T L}(\tilde{H})$ has $w \in \bar{W}_{\chi}$. Further, $w^{-1} \Phi_{M, \chi}^{+}=w^{-1} \Phi_{L}^{+} \subset \Phi_{H}^{+}=\Phi_{\chi}^{+}$. Now, the only simple root in $\Pi_{M}$ which is not in $\Phi_{M, \chi}^{+}$is

$$
\alpha_{n_{1}}= \begin{cases}e_{n_{1}}-e_{n_{1}+1}, & \text { if } n_{1}<n \\ 2 e_{n}, & \text { if } n_{1}=n .\end{cases}
$$

There are two possibilities: $w^{-1} \alpha_{n_{1}}>0$ or $w^{-1} \alpha_{n_{1}}<0$. If $w^{-1} \alpha_{n_{1}}>0$, then $w^{-1} \Pi_{M} \subset \Phi^{+}$, implying $w \in \bar{W}^{T M}$, as needed. If $w^{-1} \alpha_{n_{1}}<0$, we claim $w^{-1} c_{n_{1}} \alpha_{n_{1}}>0$. (This is clear for $n_{1}=n$. Suppose $n_{1}<n$. Observe that $w \in \bar{W}_{\chi}$ implies $w e_{n_{1}}= \pm e_{j}$ with $j \leq n_{1}$ and $w e_{n_{1}+1}= \pm e_{k}$ with $k>n_{1}$. Further, since $w \in \bar{W}^{T L}, w\left(2 e_{n_{1}+1}\right)>0$, so $w e_{n_{1}+1}=e_{k}$. The claim follows.) Also $c_{n_{1}}\left(\Pi_{M}-\left\{\alpha_{n_{1}}\right\}\right) \subset c_{n_{1}} \Phi_{M, \chi}^{+} \subset \Phi_{M, \chi}^{+}$(noting that $\left.c_{n_{1}} \in \bar{W}(\tilde{L})=\bar{W}_{\chi}(M)\right)$. Thus, $w^{-1} c_{n_{1}} \Pi_{M} \subset \Phi^{+}$, implying $c_{n_{1}} w \in \bar{W}^{T M}$, as needed. Therefore, $\bar{W}^{T L}(\tilde{H})=\bar{W}_{\chi}^{T M} \cup c_{n_{1}} \bar{W}_{\chi}^{T M}$.

Since $\left|\bar{W}_{\chi}^{T M}\right|=|\bar{W}(\tilde{H})| /|\bar{W}(\tilde{L})|$, the first property is equivalent to $\bar{W}(\tilde{L}) \bar{W}_{\chi}^{T M}=\bar{W}(\tilde{H})$. We calculate:

$$
\begin{aligned}
\bar{W}(\tilde{L}) \bar{W}_{\chi}^{T M} & =\left(\bar{W}(L) \cup \bar{W}(L) c_{n_{1}}\right) \bar{W}_{\chi}^{T M} \\
& =\bar{W}(L)\left(\bar{W}_{\chi}^{T M} \cup c_{n_{1}} \bar{W}_{\chi}^{T M}\right) \\
& =\bar{W}(L) \bar{W}^{T L}(\tilde{H}) .
\end{aligned}
$$

That $\bar{W}(L) \bar{W}^{T L}(\tilde{H})=\bar{W}(\tilde{H})$ follows easily from $\bar{W}(L) \bar{W}^{T L}(H)=\bar{W}(H)$.

The second property follows immediately from $\bar{W}_{\chi}(M) \subset \bar{W}(M)$ and $\bar{W}_{\chi}^{T M} \subset \bar{W}^{T M}$.

To check the third property, write $x=x_{H} c_{x}, w=c_{w} w_{H}$ with $c_{x}, c_{w} \in C_{\chi}$ and $x_{H}, w_{H} \in \bar{W}(H)$. Then since $l_{\tilde{H}}\left(c_{n_{1}}\right)=0$, we have $l_{\tilde{H}}(x)=l_{\tilde{H}}\left(x_{H}\right)$, $l_{\tilde{H}}(w)=l_{\tilde{H}}\left(w_{H}\right)$. Set $x_{H}^{\prime}=\left(c_{x} c_{w}\right)^{-1} x_{H}\left(c_{x} c_{w}\right)$. Then $l_{\tilde{H}}\left(x_{H}^{\prime}\right)=l_{\tilde{H}}(x)$. Since $x_{H} \in \bar{W}(L)$ and $w_{H} \in \bar{W}^{T L}(H)$, we have $l_{\tilde{H}}\left(x_{H}^{\prime}\right)+l_{\tilde{H}}\left(w_{H}\right)=$ $l_{\tilde{H}}\left(x_{H}^{\prime} w_{H}\right)=l_{\tilde{H}}\left(c_{x} c_{w} x_{H}^{\prime} w_{H}\right)=l_{\tilde{H}}(x w)$. The result follows. 
The case of $G=S O(2 n+1)$ is much easier; since $H=\tilde{H}$ is connected, the argument is just that of Case 1 above.

Lemma 3.2.8. Suppose $M$ is a standard Levi for $G, \tilde{L}$ the corresponding subgroup of $\tilde{H}$. If $\nu \hookrightarrow \operatorname{Ind}_{B_{M}}^{M} \lambda \chi$ has space $V_{\nu}^{I_{M}, \rho_{M}} \subset V_{\operatorname{Ind}_{B_{M}}^{M} \lambda \chi}^{I_{M}, \rho_{M}}$, let $V_{\nu^{\prime}}^{I_{L}^{\prime}} \subset$ $V_{\operatorname{Ind}_{B_{L}}^{\tilde{L}_{L}}}^{I_{L}^{\prime}}$ be its image under $\mathfrak{M}_{M}$ (with $\nu^{\prime}$ denoting the restriction of $\operatorname{Ind}_{B_{L}}^{\tilde{L}} \lambda$ to $\left.V_{\nu^{\prime}}^{I_{L}^{\prime}}\right)$. Then the image of $V_{\operatorname{Ind}_{P_{M}}^{I, \rho}{ }^{I}} \subset V_{\pi}^{I, \rho}$ under $\mathfrak{M}$ is $V_{\operatorname{Ind}_{P_{\tilde{L}}^{\tilde{H}}}^{I^{\prime}} \nu^{\prime}} \subset V_{\pi^{\prime}}^{I^{\prime}}$.

Remark 3.2.1. Certainly, the restriction of $\pi^{\prime}$ to $\mathfrak{M} V_{\operatorname{Ind}_{P_{M}}^{I}{ }^{I}, \rho}$ is equivalent to $\operatorname{Ind}_{P_{\tilde{L}}}^{\tilde{H}} \nu^{\prime}$. But for our purposes, it is necessary to know that the subspaces actually match up.

Proof of Lemma 3.2.8. First we need to identify the subspaces for $\operatorname{Ind}_{P_{M}}^{G} \nu$ and $\operatorname{Ind}_{P_{\tilde{L}}}^{\tilde{H}} \nu^{\prime}$. Suppose $\left\{f_{i}^{M}\right\}_{i=1, \ldots, k}=\left\{\sum_{x \in \bar{W}_{\chi}(M)} b_{x}^{i} f_{x}^{M}\right\}_{i=1, \ldots, k}$ is a basis for $V_{\nu}^{I_{M}, \rho_{M}} \subset V_{\operatorname{Ind}_{B_{M}}^{M} \lambda \chi}^{I_{M}, \rho_{M}}$. Let $f_{i}=\sum_{x \in \bar{W}_{\chi}(M)} b_{x}^{i} f_{x} \in V_{\pi}^{I, \rho}$. Then a basis for $V_{\operatorname{Ind}_{P_{M}}^{I, \rho} \lambda}^{I, \rho} V_{\pi}^{I, \rho}$ is

$$
\left\{\pi\left(T_{w^{-1}}\right) f_{i}\right\}_{\substack{i=1, \ldots, k \\ w \in \bar{W}_{\chi}^{T M}}}=\left\{\sum_{x \in \bar{W}_{\chi}(M)} b_{x}^{i} f_{x w}\right\}_{\substack{i=1, \ldots, k \\ w \in \bar{W}_{\chi}^{T M}}},
$$

with $\bar{W}_{\chi}^{T M}$ as in the preceding lemma. The proof of this is essentially the same as that of [Ja1, Lemma 2.1.4] (noting that $\bar{W}_{\chi}^{T M}$ is a set of representatives for $\bar{W}(M) \backslash \bar{W}(G))$. Similarly, if $\left\{f_{i}^{\prime \tilde{L}^{2}}\right\}_{i=1, \ldots, j}=\left\{\sum_{x \in \bar{W}(L)} c_{x}^{i} f_{x}^{\prime \tilde{L}}\right\}_{i=1, \ldots, j}$ is a basis for $V_{\nu^{\prime}}^{I_{L}^{\prime}} \subset V_{\operatorname{Ind}_{B_{L}}^{\tilde{L}} \lambda}^{I_{L}^{\prime}}$, then $\operatorname{Ind}_{P_{\tilde{L}}}^{\tilde{H}} \nu^{\prime}$ has basis

$$
\left\{\pi^{\prime}\left(T_{w^{-1}}^{\prime}\right) f_{i}^{\prime}\right\}_{\substack{i=1, \ldots, j \\ w \in \bar{W}^{T} \bar{L}}}=\left\{\sum_{x \in \bar{W}(L)} c_{x}^{i} f_{x w}^{\prime}\right\}_{\substack{i=1, \ldots, j \\ w \in \bar{W}^{T \bar{L}}}} .
$$

Here we are taking $\bar{W}^{T \tilde{L}}=\bar{W}_{\chi}^{T M}$. (By the preceding lemma, $\bar{W}_{\chi}^{T M}$ has the properties we need; we change notation only for appearance's sake.)

We now check how subspaces match up. First observe that $\mathfrak{M}: f_{w} \longmapsto$ $\pi^{\prime}\left(\Psi\left(T_{w^{-1}}\right) \Psi\left(T_{w_{0}^{-1}}^{-1}\right)\right) f_{w_{0}}^{\prime}$. Therefore, if $\Psi: T_{w} \longmapsto a_{w} T_{w}^{\prime}$, we see that $\mathfrak{M} f_{w}=$ $m(w) f_{w}^{\prime}$, where $m(w)=a_{w^{-1}} a_{w_{0}^{-1}}^{-1}$. Similarly, using Proposition 1.6, we get $\mathfrak{M}_{M} f_{w}^{M}=m_{M}(w) f_{w}^{\prime \tilde{L}}$ with $m_{M}(w)=a_{w^{-1}} a_{w_{0, M}^{-1}}^{-1}\left(w_{0, M}\right.$ the longest element 
of $\left.\bar{W}_{\chi}(M)\right)$. If $\left\{f_{i}^{M}\right\}_{i=1, \ldots, k}$ as above is a basis for $V_{\nu}^{I_{M}, \rho_{M}}$, then $V_{\operatorname{Ind}_{P_{M}}^{G} \lambda}^{I, \rho}$ has basis $\left\{\sum_{x \in \bar{W}_{\chi}(M)} b_{x}^{i} f_{x w}\right\}_{\substack{i=1, \ldots, k \\ w \in \bar{W}_{\chi}^{T M}}}$. Therefore, $\mathfrak{M} V_{\operatorname{Ind}_{P_{M}}^{I, \rho}}^{I, \rho}$ has basis (using $\left.\bar{W}_{\chi}(M)=\bar{W}(\tilde{L}), \bar{W}_{\chi}^{T M}=\bar{W}^{T \tilde{L}}\right)$

$$
\left\{\sum_{x \in \bar{W}(\tilde{L})} b_{x}^{i} \pi^{\prime}\left(\Psi\left(T_{w^{-1} x^{-1}}\right) \Psi\left(T_{w_{0}^{-1}}^{-1}\right)\right) f_{w_{0}}^{\prime}\right\}_{\substack{i=1, \ldots, k \\ w \in \bar{W}^{T} \tilde{L}}} .
$$

On the other hand, $V_{\nu^{\prime}}^{I_{L}^{\prime}}$ has basis

$$
\left\{\mathfrak{M}_{M} f_{i}^{M}\right\}_{i=1, \ldots, k}=\left\{\sum_{x \in \bar{W}(\tilde{L})} b_{x}^{i} m_{M}(x) f_{x}^{\prime \tilde{L}}\right\}_{i=1, \ldots, k} .
$$

Therefore, $V_{\operatorname{Ind}_{P_{\tilde{L}}}^{\tilde{H}} \nu^{\prime}}^{I^{\prime}}$ has basis

$$
\left\{\sum_{x \in \bar{W}(\tilde{L})} b_{x}^{i} m_{M}(x) f_{x w}^{\prime}\right\}_{\substack{i=1, \ldots, k \\ w \in \bar{W}^{T \tilde{L}}}} .
$$

Finally, by Lemma 3.2.7, for $x \in \bar{W}_{\chi}(M)$ and $w \in \bar{W}_{\chi}^{T M}$, we have $T_{w^{-1} x^{-1}}=$ $T_{w^{-1}} T_{x^{-1}}$ and $T_{w^{-1} x^{-1}}^{\prime}=T_{w^{-1}}^{\prime} T_{x^{-1}}^{\prime}$. From this, one sees that $a_{w^{-1} x^{-1}}=$ $a_{w^{-1}} a_{x^{-1}}$. Therefore, $m(x w)=\left(a_{w^{-1}} \frac{a_{w_{0, M}^{-1}}}{a_{w_{0}^{-1}}}\right) m_{M}(x)$. The conclusion follows.

Let $R\left(w_{\mathfrak{p}}, \lambda_{\mathfrak{p}}, \chi\right)$ denote the normalized standard intertwining operator defined earlier. Since $w_{\mathfrak{p}} \in \bar{W}_{\chi}$, we may identify $w_{\mathfrak{p}} \in \bar{W}^{\prime}$ with $w_{\mathfrak{p}_{1}}$. $w_{\mathfrak{p}_{0}} \in \bar{W}\left(H_{1}^{\prime}\right) \times \bar{W}\left(H_{0}^{\prime}\right)\left(\mathfrak{p}_{1}, \mathfrak{p}_{0}\right.$ are ordered partitions for $\left.H_{1}^{\prime}, H_{0}^{\prime}\right)$. If we use $H^{\prime}=H_{1}^{\prime} \times H_{0}^{\prime}$ to identify the degenerate principal series $I_{H^{\prime}}\left(\lambda_{\mathfrak{p}}, 1\right)$ with $I_{H_{1}^{\prime}}\left(\lambda_{\mathfrak{p}_{1}}, 1\right) \otimes I_{H_{0}^{\prime}}\left(\lambda_{\mathfrak{p}_{0}}, 1\right)$, we have

$$
R^{\prime}\left(w_{\mathfrak{p}}, \lambda_{\mathfrak{p}}, 1\right)=R_{1}^{\prime}\left(w_{\mathfrak{p}_{1}}, \lambda_{\mathfrak{p}_{1}}, 1\right) \otimes R_{0}^{\prime}\left(w_{\mathfrak{p}_{0}}, \lambda_{\mathfrak{p}_{0}}, 1\right)
$$

for the corresponding intertwining operators.

Proposition 3.2.9. $R\left(w_{\mathfrak{p}}, \lambda_{\mathfrak{p}}, \chi\right)$ is a non-zero multiple of

$$
\mathfrak{M}^{-1} \circ R_{1}^{\prime}\left(w_{\mathfrak{p}_{1}}, \lambda_{\mathfrak{p}_{1}}, 1\right) \otimes R_{0}^{\prime}\left(w_{\mathfrak{p}_{0}}, \lambda_{\mathfrak{p}_{0}}, 1\right) \circ \mathfrak{M}
$$

Proof. We argue as in $[\mathbf{R e} 2]$. Let $x=\left(x_{1}, \ldots, x_{s}\right), y=\left(y_{1}, \ldots, y_{t+1}\right)$ and set

$$
\lambda_{\mathfrak{p}_{1}}+x=|\operatorname{det}|^{\frac{a_{1}-b_{1}}{4}+x_{1}} \times \cdots \times|\operatorname{det}|^{\frac{a_{s}-b_{s}}{4}+x_{s}}
$$




$$
\lambda_{\mathfrak{p}_{0}}+y=|\operatorname{det}|^{\frac{c_{1}-d_{1}}{4}+y_{1}} \times \cdots \times|\operatorname{det}|^{\frac{c_{t}-d_{t}}{4}+y_{t}} \times|\operatorname{det}|^{\frac{c_{t+1}}{4}+y_{t+1}}
$$

and $\lambda_{\mathfrak{p}}+(x, y)=\left(\lambda_{\mathfrak{p}_{1}}+x\right) \times\left(\lambda_{\mathfrak{p}_{0}}+y\right)$. Since $\bar{I}\left(\lambda_{\mathfrak{p}}+(x, y), \chi\right)$ and $\bar{I}_{H_{1}^{\prime}}^{\prime}\left(\lambda_{\mathfrak{p}_{1}}+\right.$ $x, 1), \bar{I}_{H_{0}^{\prime}}^{\prime}\left(\lambda_{\mathfrak{p}_{0}}+y, 1\right)$ are irreducible representations for $(x, y) \neq(0,0)$ near zero, Schur's lemma for $p$-adic groups tells us

$$
\begin{aligned}
& R\left(w_{\mathfrak{p}}, \lambda_{\mathfrak{p}}+(x, y), \chi\right) \\
& =c(x, y) \mathfrak{M}^{-1} \circ R_{1}^{\prime}\left(w_{\mathfrak{p}_{1}}, \lambda_{\mathfrak{p}_{1}}+x, 1\right) \otimes R_{0}^{\prime}\left(w_{\mathfrak{p}_{0}}, \lambda_{\mathfrak{p}_{0}}+y, 1\right) \circ \mathfrak{M}
\end{aligned}
$$

for such $(x, y)(c(x, y)$ scalar). Now, observe that $\mathfrak{M}$ is independent of $(x, y)$. By Proposition 3.1, Lemma 3.2 and [M1], $R\left(w_{\mathfrak{p}}, \lambda_{\mathfrak{p}}+(x, y), \chi\right)$ and $\mathfrak{M}^{-1} \circ R_{1}^{\prime}\left(w_{\mathfrak{p}_{1}}, \lambda_{\mathfrak{p}_{1}}+x, 1\right) \otimes R_{0}^{\prime}\left(w_{\mathfrak{p}_{0}}, \lambda_{\mathfrak{p}_{0}}+y, 1\right) \circ \mathfrak{M}$ are both holomorphic and non-zero at $(x, y)=(0,0)$. By analytic continuation, $(*)$ holds at $(x, y)=$ $(0,0)$. Further, we see that $c(x, y)$ must be holomorphic and non-vanishing at $(x, y)=(0,0)$. The proposition follows.

The results above begin the process of separating the effects of the four characters of order $\leq 2$, essentially allowing us to deal with $1, \mu_{n r}$ separately from $\mu, \mu \mu_{n r}$ (with $\mu, \mu_{n r}$ associated to $1, \mu_{n r}$ for $H_{1}^{\prime}$ ). To finish this process, we need to separate the effects of 1 and $\mu_{n r}$ for both symplectic and orthogonal groups. To do this, we use an argument similar to that above, only with $[\mathbf{B}-\mathbf{M o 2}]$ playing the role that $[\mathbf{R}]$ played above. We give the argument for the symplectic case; the orthogonal case is similar.

Let $\chi=(\underbrace{\mu_{n r}, \ldots, \mu_{n r}}_{n_{1}}, \underbrace{1, \ldots, 1}_{n_{0}})$ and $\lambda=||^{x_{1}} \times \cdots \times||^{x_{n_{1}}} \times\left.||\right|^{y_{1}} \times \cdots \times||^{y_{n_{0}}}$ with $x_{1}, \ldots, x_{n_{1}}, y_{1}, \ldots, y_{n_{0}} \in \mathbb{R}$. Again, set $H^{\prime}=H_{1}^{\prime} \times H_{0}^{\prime}$. Let $\pi=$ $\operatorname{Ind}_{B}^{G}(\lambda \chi)$ and $\pi^{\prime}=\operatorname{Ind}_{B^{\prime}}^{H^{\prime}}(\lambda)=\left(\operatorname{Ind}_{B_{1}^{\prime}}^{H_{1}^{\prime}} \lambda_{1}\right) \otimes\left(\operatorname{Ind}_{B_{0}^{\prime}}^{H_{0}^{\prime}} \lambda_{0}\right)=\pi_{1}^{\prime} \otimes \pi_{0}^{\prime}$ (with $\lambda_{1}=||^{x_{1}} \times \cdots \times||^{x_{n_{1}}}$ and $\left.\lambda_{0}=||^{y_{1}} \times \cdots \times||^{y_{n_{0}}}\right)$. We let $\tau, \tau^{\prime}, \tau_{1}^{\prime}, \tau_{0}^{\prime}$ denote the infinitesimal characters of $\pi, \pi^{\prime}, \pi_{1}^{\prime}, \pi_{0}^{\prime}$, resp.

We define a map $\mathfrak{M}_{\tau}: V_{\pi}^{I} \longrightarrow V_{\pi^{\prime}}^{I^{\prime}}$ (unlike the situation above, $\mathfrak{M}_{\tau}$ is not independent of $\lambda$ ). Let $\mathfrak{M}_{\tau}: f_{w_{0}} \longmapsto f_{w_{0}}^{\prime}$. If $q, q^{\prime}$ denote the quotient maps $q: \mathcal{H}(G) \longrightarrow \mathcal{H}_{\tau}(G), q^{\prime}: \mathcal{H}\left(H^{\prime}\right) \longrightarrow \mathcal{H}_{\tau^{\prime}}\left(H^{\prime}\right)$ (cf. [B-Mo2, p. 619]), then we certainly have $f_{w_{0}}, f_{w_{0}}^{\prime}$ generating $V_{\pi}^{I}, V_{\pi^{\prime}}^{I^{\prime}}$, under the action of $\pi(q(\mathcal{H}(K))), \pi^{\prime}\left(q^{\prime}\left(\mathcal{H}\left(K^{\prime}\right)\right)\right)$. Thus, as above, we may extend $\mathfrak{M}_{\tau}$ to get a linear isomorphism satisfying $\pi^{\prime}\left(\Psi_{\tau}(h)\right)\left(\mathfrak{M}_{\tau} f\right)=\mathfrak{M}_{\tau}(\pi(h) f)$ for all $h \in q(\mathcal{H}(K)), f \in V_{\pi}^{I}$. Here $\Psi: \mathcal{H}_{\tau}(G) \longrightarrow \mathcal{H}_{\tau^{\prime}}\left(H^{\prime}\right)$ is the isomorphism of quotient algebras obtained by composing the isomorphisms in [B-Mo2]. With notation as above, we have the following:

Lemma 3.2.10. $\mathfrak{M}_{\tau}$ has the following properties:

(1) $\pi^{\prime}\left(\Psi_{\tau}(h)\right)\left(\mathfrak{M}_{\tau} f\right)=\mathfrak{M}_{\tau}(\pi(h) f)$ for all $h \in \mathcal{H}(G), f \in V_{\pi}^{I}$.

(2) $\mathfrak{M}_{\tau}$ is real analytic in $x_{1}, \ldots, x_{n_{1}}, y_{1}, \ldots, y_{n_{0}}$. 
Proof. The proof of (1) is similar to Proposition 3.2.6 above. For $h \in$ $q(\mathcal{H}(K))$, it holds by definition. For $t, t^{\prime}$ corresponding to the same element of $Y$, we have that $\Psi_{\tau}: \delta^{\frac{1}{2}}(t) q\left(T_{t}\right) \longmapsto \chi(t) \delta^{\frac{1}{2}}\left(t^{\prime}\right) q^{\prime}\left(T_{t^{\prime}}\right)$ (a consequence of [B-Mo2, (4.4)] and the construction of $\Psi_{\tau}$ ). With this observation, the rest of (1) follows as in the proof of Proposition 3.2.6.

For (2), if $w \in \bar{W}_{\chi}=\bar{W}\left(H^{\prime}\right)$, we have

$$
\mathfrak{M}_{\tau}\left(f_{w}\right)=\pi^{\prime}\left(\Psi_{\tau}\left(q\left(T_{w}\right)\right)\right) f_{w_{0}}^{\prime} .
$$

It is sufficient to show $\Psi_{\tau}\left(q\left(T_{w}\right)\right)$ is analytic, which follows from [B-Mo2, Theorem 4.3].

Let $R\left(w_{\mathfrak{p}}, \lambda_{\mathfrak{p}}, \chi\right)$ be the normalized standard intertwining operator defined earlier. As before, we can write $w_{\mathfrak{p}}=w_{\mathfrak{p}_{1}} w_{\mathfrak{p}_{0}} \in \bar{W}\left(H_{1}^{\prime}\right) \times \bar{W}\left(H_{0}^{\prime}\right)$, and $\mathfrak{p}_{i}$ is an ordered partition for $H_{i}^{\prime}$. We decompose $\lambda_{\mathfrak{p}}=\lambda_{\mathfrak{p}_{1}} \times \lambda_{\mathfrak{p}_{0}}$ as above. Let $R^{\prime}\left(w_{\mathfrak{p}}, \lambda_{\mathfrak{p}}, 1\right)=R^{\prime}\left(w_{\mathfrak{p}_{1}}, \lambda_{\mathfrak{p}_{1}}, 1\right) \otimes R^{\prime}\left(w_{\mathfrak{p}_{0}}, \lambda_{\mathfrak{p}_{0}}, 1\right)$ be the corresponding intertwining operator for $H^{\prime}$.

Proposition 3.2.11. $R\left(w_{\mathfrak{p}}, \lambda_{\mathfrak{p}}, \chi\right)=\mathfrak{M}_{\tau} \circ R^{\prime}\left(w_{\mathfrak{p}_{1}}, \lambda_{\mathfrak{p}_{1}}, 1\right) \otimes R^{\prime}\left(w_{\mathfrak{p}_{0}}, \lambda_{\mathfrak{p}_{0}}, 1\right) \circ$ $\mathfrak{M}_{\tau}^{-1}$.

Proof. We again argue as in $[\mathbf{R e} 2]$. With notation as in the proof of Proposition 3.2.9, we again have that if $(x, y) \neq 0$ near 0 ,

$$
\begin{aligned}
& R\left(w_{\mathfrak{p}}, \lambda_{\mathfrak{p}}+(x, y), \chi\right) \\
& =\mathfrak{M}_{\tau(x, y)} \circ R^{\prime}\left(w_{\mathfrak{p}_{1}}, \lambda_{\mathfrak{p}_{1}}+x, 1\right) \otimes R^{\prime}\left(w_{\mathfrak{p}_{0}}, \lambda_{\mathfrak{p}_{0}}+y, 1\right) \circ \mathfrak{M}_{\tau(x, y)}^{-1},
\end{aligned}
$$

where $\tau(x, y)$ is the infinitesimal character associated to $\operatorname{Ind}_{B}^{G}\left(\lambda_{\mathfrak{p}}+(x, y)\right) \chi$. In this case, there is no need to introduce a scalar $c(x, y)$-the action of $(* *)$ on $K$-fixed vectors tells us we actually have equality. By Proposition 3.1 and the preceding lemma, both sides of $(* *)$ are analytic in $(x, y)$. Therefore by analytic continuation, $(* *)$ holds at $(0,0)$, as needed.

Remark 3.2.2. Hecke algebras are not available for archimedean places. Also Roche's results are not available for the place $v, v \mid 2$.

Remark 3.2.3. Above, we have used the results of Barbasch-Moy and Roche to identify $\operatorname{Ind}_{B}^{G} \lambda \chi$ with $\operatorname{Ind}_{B_{1}^{\prime}}^{G_{1}^{\prime}} \lambda_{1} \otimes \operatorname{Ind}_{B_{2}^{\prime}}^{G_{2}^{\prime}} \lambda_{2} \otimes \operatorname{Ind}_{B_{3}^{\prime}}^{G_{3}^{\prime}} \lambda_{3} \otimes \operatorname{Ind}_{B_{0}^{\prime}}^{G_{0}^{\prime}} \lambda_{0}$, where $\chi=(\underbrace{\mu, \ldots, \mu}_{r_{1}}, \underbrace{\mu \mu_{n r}, \ldots, \mu \mu_{n r}}_{r_{2}}, \underbrace{\mu_{n r}, \ldots, \mu_{n r}}_{r_{3}}, \underbrace{1, \ldots, 1}_{r_{0}})$ and $\lambda=\lambda_{1} \times$ $\lambda_{2} \times \lambda_{3} \times \lambda_{0}$. The same isomorphisms allow us to identify $\operatorname{Ind}_{B_{1}^{\prime}}^{G_{1}^{\prime}} \lambda_{1}$ with $\operatorname{Ind}_{B_{1}}^{G_{1}} \lambda_{1} \chi_{1}$, where $\chi_{1}=(\underbrace{\mu, \ldots, \mu}_{r_{1}})$ and $G_{1}=G\left(r_{1}\right)$ and similarly for $G_{2}^{\prime}, G_{3}^{\prime}$. Thus, we may also identify $\operatorname{Ind}_{B}^{G} \lambda \chi$ with $\operatorname{Ind}_{B_{1}}^{G_{1}} \lambda_{1} \chi_{1} \otimes \operatorname{Ind}_{B_{2}}^{G_{2}} \lambda_{2} \chi_{2} \otimes$ $\operatorname{Ind}_{B_{3}}^{G_{3}} \lambda_{3} \chi_{3} \otimes \operatorname{Ind}_{B_{0}^{\prime}}^{G_{0}^{\prime}} \lambda_{0}$ (with $G_{0}=G_{0}^{\prime}$ ). This correspondence is done in 
general in [Ja3]. However, there are two basic obstacles to using [Ja3] here. The first is that, as with $[\mathbf{A u 1}],[\mathbf{A u 2}],[\mathbf{S c - S t}]$, the results in $[\mathbf{J a 3}]$ are done in the Grothendieck group setting, hence do not deal with composition series. The second is that we deal with $\operatorname{Ind}_{B_{1}^{\prime}}^{G_{1}^{\prime}} \lambda_{1}$, etc., by using Mœglin's results. To work with $\operatorname{Ind}_{B_{1}}^{G_{1}} \lambda_{1} \chi_{1}$, etc., we would have to establish the corresponding results ourselves.

To reduce our problem to that covered by Møglin's results, we will also need the following proposition. (We continue to use $j$ for the IwahoriMatsumoto involution.)

Proposition 3.2.12. Suppose $\pi$ and $\pi^{\prime}=\pi_{1}^{\prime} \times \pi_{2}^{\prime} \times \pi_{3}^{\prime} \times \pi_{0}^{\prime}$ are corresponding irreducible representations. Then, $j(\pi)$ is tempered (resp., squareintegrable) if and only if $j\left(\pi_{1}^{\prime}\right), j\left(\pi_{2}^{\prime}\right), j\left(\pi_{3}^{\prime}\right), j\left(\pi_{0}^{\prime}\right)$ are all tempered (resp., square-integrable).

Proof. Note that $j\left(\pi^{\prime}\right)$ is tempered (resp., square-integrable) if and only if $j\left(\pi_{1}^{\prime}\right), j\left(\pi_{2}^{\prime}\right), j\left(\pi_{3}^{\prime}\right), j\left(\pi_{0}^{\prime}\right)$ are all tempered (resp., square-integrable).

Let us write $\theta \in \operatorname{Jac}(\pi)$ if $\theta$ appears in the normalized Jacquet module of $\pi$ (with respect to the Borel subgroup) with multiplicity at least one. Observe that by the abelianness of $T$ and Frobenius reciprocity, we have $\theta \in \operatorname{Jac}(\pi)$ if and only if $\pi \hookrightarrow \operatorname{Ind}_{B}^{G} \theta$. Therefore, by Theorem 1.8 and Frobenius reciprocity, we see that $\theta \in \operatorname{Jac}(\pi)$ if and only if $\theta^{-1} \in \operatorname{Jac}(j(\pi))$. Similarly, $\theta_{i} \in \operatorname{Jac}\left(\pi_{i}^{\prime}\right)$ if and only if $\theta_{i}^{-1} \in \operatorname{Jac}\left(j\left(\pi_{i}^{\prime}\right)\right)$.

For notational convenience, let $\mu^{(k)}=\underbrace{\mu \times \cdots \times \mu}_{k}$. Let $\mu_{1}=\mu, \mu_{2}=\mu \mu_{n r}$, $\mu_{3}=\mu_{n r}, \mu_{0}=1$, as above. We claim that $\lambda_{1} \mu_{1}^{\left(r_{1}\right)} \times \lambda_{2} \mu_{2}^{\left(r_{2}\right)} \times \lambda_{3} \mu_{3}^{\left(r_{3}\right)} \times$ $\lambda_{0} \mu_{0}^{\left(r_{0}\right)} \in \operatorname{Jac}(\pi)$ if and only if $\lambda_{i} \in \operatorname{Jac}\left(\pi_{i}^{\prime}\right)$ for $i=0,1,2,3$. First, let $G_{0}^{\prime \prime}=G\left(r_{3}+r_{0}\right)$ and

$$
G_{1}^{\prime \prime}= \begin{cases}O\left(2\left(r_{1}+r_{2}\right)\right), & \text { if } G=S p(2 n), O(2 n) \\ S O\left(2\left(r_{1}+r_{2}\right)+1\right), & \text { if } G=S O(2 n+1) .\end{cases}
$$

Suppose that $\pi$ corresponds to $\pi_{1}^{\prime \prime} \times \pi_{0}^{\prime \prime}$ under Roche's isomorphism. We then argue as follows: $\left(\lambda_{1} \mu_{1}^{\left(r_{1}\right)} \times \lambda_{2} \mu_{2}^{\left(r_{2}\right)}\right) \times\left(\lambda_{3} \mu_{3}^{\left(r_{3}\right)} \times \lambda_{0} \mu_{0}^{\left(r_{0}\right)}\right) \in \operatorname{Jac}(\pi)$ if and only if $\pi \hookrightarrow \operatorname{Ind}_{B}^{G}\left(\left(\lambda_{1} \mu_{1}^{\left(r_{1}\right)} \times \lambda_{2} \mu_{2}^{\left(r_{2}\right)}\right) \times\left(\lambda_{3} \mu_{3}^{\left(r_{3}\right)} \times \lambda_{0} \mu_{0}^{\left(r_{0}\right)}\right)\right)$ if and only if $\pi_{1}^{\prime \prime} \hookrightarrow \operatorname{Ind}_{B_{1}^{\prime \prime}}^{G^{\prime \prime}}\left(\lambda_{1} \times \lambda_{2} \mu_{n r}^{\left(r_{2}\right)}\right)$ and $\pi_{0}^{\prime \prime} \hookrightarrow \operatorname{Ind}_{B_{0}^{\prime \prime}}^{G_{0}^{\prime \prime}}\left(\lambda_{3} \mu_{n r}^{\left(r_{3}\right)} \times \lambda_{0}\right)$ (cf. [R, Theorem 9.5]) if and only if $\lambda_{1} \times \lambda_{2} \mu_{n r}^{\left(r_{2}\right)} \in \operatorname{Jac}\left(\pi_{1}^{\prime \prime}\right)$ and $\lambda_{3} \mu_{n r}^{\left(r_{3}\right)} \times \lambda_{0} \in \operatorname{Jac}\left(\pi_{0}^{\prime \prime}\right)$. We use the same basic argument in conjunction with the results of Barbasch-Moy, making a few minor modifications to cover induction via tensor product. We argue as follows for $\pi_{1}^{\prime \prime}: \lambda_{1} \times \lambda_{2} \mu_{n r}^{\left(r_{2}\right)} \in \operatorname{Jac}\left(\pi_{1}^{\prime \prime}\right)$ if and only if $\pi_{1}^{\prime \prime} \hookrightarrow$ $\operatorname{Ind}_{B_{1}^{\prime \prime}}^{G_{1 \prime}^{\prime \prime}}\left(\lambda_{1} \times \lambda_{2} \mu_{n r}^{\left(r_{2}\right)}\right)$ if and only if $\pi_{1}^{\prime \prime} \hookrightarrow \otimes-\operatorname{Ind}_{B_{1}^{\prime \prime}}^{G^{\prime \prime}}\left(\lambda_{1}^{-1} \times \lambda_{2}^{-1} \mu_{n r}^{\left(r_{2}\right)}\right)$ if and only 
if $\pi_{1}^{\prime} \hookrightarrow \otimes-\operatorname{Ind}_{B_{1}^{\prime}}^{G_{1}^{\prime}} \lambda_{1}^{-1}$ and $\pi_{2}^{\prime} \hookrightarrow \otimes-\operatorname{Ind}_{B_{2}^{\prime}}^{G_{2}^{\prime}} \lambda_{2}^{-1}$ (cf. [B-Mo2, Theorem 6.2]) if and only if $\lambda_{1} \in \operatorname{Jac}\left(\pi_{1}^{\prime}\right)$ and $\lambda_{2} \in \operatorname{Jac}\left(\pi_{2}^{\prime}\right)$. The argument for $\pi_{0}^{\prime \prime}$ is similar. This verifies our claim.

Next we claim that any $\theta \in \operatorname{Jac}(\pi)$ has the form $\operatorname{sh}\left(\lambda_{1} \mu_{1}^{\left(r_{1}\right)}, \lambda_{2} \mu_{2}^{\left(r_{2}\right)}\right.$, $\left.\lambda_{3} \mu_{3}^{\left(r_{3}\right)}, \lambda_{0} \mu_{0}^{\left(r_{0}\right)}\right)$ for some $\lambda_{1}, \lambda_{2}, \lambda_{3}, \lambda_{0}$ with $\lambda_{i} \in \operatorname{Jac}\left(\pi_{i}^{\prime}\right), i=0,1,2,3$. Here, sh denotes a shuffle, used in the usual sense (e.g., see [Ja3, Definition 3.1]). This claim follows immediately from the discussion above and [Ja3, Lemma 5.4].

Since $\theta \in \operatorname{Jac}(\pi)$ if and only if $\theta^{-1} \in \operatorname{Jac}(j(\pi))$, the inequalities required by the Casselman criteria for $j(\pi)$ to be tempered (resp., square-integrable) have the same form as those in [M1, Remarque 1.3.5] $\left(\pi_{1}^{\prime}, \pi_{2}^{\prime}, \pi_{3}^{\prime}, \pi_{0}^{\prime}\right.$ are already covered by [M1, Remarque 1.3.5]). Further, observe that $\lambda_{1}, \lambda_{2}, \lambda_{3}, \lambda_{0}$ each satisfy the inequalities of [M1, Remarque 1.3.5] if and only if $\operatorname{sh}\left(\lambda_{1} \mu_{1}^{\left(r_{1}\right)}, \lambda_{2} \mu_{2}^{\left(r_{2}\right)}, \lambda_{3} \mu_{3}^{\left(r_{3}\right)}, \lambda_{0} \mu_{0}^{\left(r_{0}\right)}\right)$ satisfies the inequalities of [M1, Remarque 1.3.5] for every shuffle sh. The proof of this is straightforward; essentially identical to that of [Ja3, Corollary 8.3]. Thus, $j(\pi)$ is tempered (resp., square-integrable) if and only if $j\left(\pi_{1}^{\prime}\right), j\left(\pi_{2}^{\prime}\right), j\left(\pi_{3}^{\prime}\right), j\left(\pi_{0}^{\prime}\right)$ are all tempered (resp., square-integrable), as needed.

Remark 3.2.4. We may also use the above result to classify the squareintegrable (resp., tempered) representations of $S p(2 n, F), S O(2 n+1, F)$ supported on the Borel subgroup (at least for $F$ having odd residual characteristic). By [Ta3, Theorem 6.2], such a square-integrable (resp., tempered) representation has cuspidal support contained in $\left\{||^{\alpha} \mu\right\}_{\alpha \in \mathbb{R}, \mu^{2}=1}$. Now, to classify such representations, it suffices to classify the representations $\pi$ with cuspidal support in $\left\{||^{\alpha} \mu\right\}_{\alpha \in \mathbb{R}, \mu^{2}=1}$ such that $j(\pi)$ is square-integrable (resp., tempered). By the preceding proposition, it suffices to classify the corresponding representations $\pi_{1}^{\prime}, \pi_{2}^{\prime}, \pi_{3}^{\prime}, \pi_{0}^{\prime}$ of $G_{1}^{\prime}, G_{2}^{\prime}, G_{3}^{\prime}, G_{0}^{\prime}$. This is done in [M1].

3.3. The definition of $\operatorname{Unip}(\mathfrak{p}, \chi)$. We prove:

\section{Proposition 3.3.1.}

(1) $R\left(w_{\mathfrak{p}}, \lambda_{\mathfrak{p}}, \chi\right) I\left(\lambda_{\mathfrak{p}}, \chi\right)$ is semi-simple and the generalized Iwahori-Matsumoto involution of its direct summands is tempered. Let $\operatorname{Unip}(\mathfrak{p}, \chi)$ be the set of direct summands of $R\left(w_{\mathfrak{p}}, \lambda_{\mathfrak{p}}, \chi\right) I\left(\lambda_{\mathfrak{p}}, \chi\right)$.

(2) Under the Hecke algebra isomorphism, $\operatorname{Unip}(\mathfrak{p}, \chi)$ corresponds to a subset of $\operatorname{Unip}\left(O_{1}\right) \times \cdots \times \operatorname{Unip}\left(O_{k}\right) \times \operatorname{Unip}\left(O_{0}\right)$.

Proof. By Proposition 3.2.1, it is enough to prove for $\chi=1$. For simplicity, we denote $\operatorname{Unip}(\mathfrak{p}, 1)=\operatorname{Unip}(\mathfrak{p})$. Let $O$ be the unipotent orbit obtained from $\mathfrak{p}$ by ignoring the ordering: We will prove $\operatorname{Unip}(\mathfrak{p}) \subset \operatorname{Unip}(O)$, where $O$ is a unipotent orbit obtained from $\mathfrak{p}$ by ignoring the ordering.

If $\mathfrak{p}=\left(a_{1}, b_{1}, \ldots, a_{s}, b_{s}, a_{s+1}\right)$ satisfies the two conditions in Remark 3.2, then we are in Mœglin's situation. So it is clear by Mœglin's result. 
Otherwise, by Remark 3.3, such chain can be written as $\mathfrak{p}=\mathfrak{p}_{1} \times \cdots \times \mathfrak{p}_{k} \times$ $\mathfrak{p}_{0}$, where $\mathfrak{p}_{i} \in P\left(O_{i}\right)$ and $O_{i}$ is a distinguished unipotent orbit in $G_{i}^{*}$ for $i=$ $0,1, \ldots, k$. Let $\mu_{i}, i=1, \ldots, k$ be non-trivial quadratic grössencharacters such that $\mu_{i v}=1$ for $i=1, \ldots, k$ for a given non-archimedean place $v$. Let $\chi=\chi(\underbrace{\mu_{1}, \ldots, \mu_{1}}_{r_{1}}, \ldots, \underbrace{\mu_{k}, \ldots, \mu_{k}}_{r_{k}}, \underbrace{1, \ldots, 1}_{r_{0}})$.

Consider the pseudo-Eisenstein series attached to $\chi$ from [Ki3]:

$$
\begin{aligned}
& l_{\mathfrak{p}}(\phi, \lambda, \chi)=\prod_{i=0}^{k} \sum_{w_{i} \in W_{i}} r\left(w_{i},-\lambda_{i}, \Phi_{i}\right) R\left(w_{\mathfrak{p}} w_{k}^{-1} \cdots w_{0}^{-1}, w_{0} \cdots w_{k} \lambda, \chi\right) \\
& \sum_{d \in D} r\left(d w_{1} \cdots w_{k} w_{0},-\lambda, \Phi_{D}\right) R\left(d^{-1}, d w_{1} \cdots w_{k} w_{0} \lambda, d \chi\right) \phi\left(d w_{1} \cdots w_{k} w_{0} \lambda\right),
\end{aligned}
$$

where $\Phi_{i}$ 's are given by:

$$
\begin{aligned}
\Phi_{1}= & \left\{e_{i} \pm e_{j}, \quad 1 \leq i<j \leq r_{1}\right\} \\
\Phi_{2}= & \left\{e_{r_{1}+i} \pm e_{r_{1}+j}, \quad 1 \leq i<j \leq r_{2}\right\} \\
& \vdots \\
\Phi_{k}= & \left\{e_{r_{1}+\cdots+r_{k-1}+i} \pm e_{r_{1}+\cdots+r_{k-1}+j}, \quad 1 \leq i<j \leq r_{k}\right\} \\
\Phi_{0}=\left\{e_{r_{1}+\cdots+r_{k}+i} \pm e_{r_{1}+\cdots+r_{k}+j,},\right. & \left.1 \leq i<j \leq r_{0}, \quad 2 e_{r_{1}+\cdots+r_{k}+i}, \quad i=1, \ldots, r_{0}\right\} \\
& \quad 1 \leq i, \\
\Phi_{D}= & \Phi^{+}-\bigcup_{i=0}^{k} \Phi_{k} .
\end{aligned}
$$

We note that the above is for $G=S p(2 n)$. If $G=S O(2 n+1)$, we need to add, to $\Phi_{i}, e_{r_{1}+\cdots+r_{i-1}+j}, \quad j=1, \ldots, r_{i}$, for $i=1, \ldots, k$ and in $\Phi_{0}$, $2 e_{r_{1}+\cdots+r_{k}+i}$ should be $e_{r_{1}+\cdots+r_{k}+i}$. If $G=O(2 n)$, then $\Phi_{0}$ does not have the roots $2 e_{r_{1}+\cdots+r_{k}+i}, \quad i=1, \ldots, r_{0}$.

Also $D$ is the set of distinguished coset representatives for $\theta=\Delta-$ $\left\{e_{r_{1}}-e_{r_{1}+1}, e_{r_{1}+r_{2}}-e_{r_{1}+r_{2}+1}, \ldots, e_{r_{1}+\cdots+r_{k}}-e_{r_{1}+\cdots+r_{k}+1}\right\} \subset \Delta=\left\{e_{1}-\right.$ $\left.e_{2}, \ldots, e_{n-1}-e_{n}\right\}$ and $W_{i}$ is the Weyl group of $G_{i}^{\prime}$ for $i=0,1, \ldots, k$. Let $\lambda=\lambda_{1}+\cdots+\lambda_{k}+\lambda_{0}$, where $\lambda_{i}=a_{r_{1}+\cdots+r_{i-1}+1} e_{r_{1}+\cdots+r_{i-1}+1}+\cdots+$ $a_{r_{1}+\cdots+r_{i}} e_{r_{1}+\cdots+r_{i}}$ for $i=1, \ldots, k$ and $\lambda_{0}=a_{r_{1}+\cdots+r_{k}+1} e_{r_{1}+\cdots+r_{k}+1}+\cdots+$ $a_{n} e_{n}$.

Now we substitute $\chi=1$ and we show that $l_{\mathfrak{p}}\left(\phi, \lambda_{\mathfrak{p}}, \chi=1\right)$ is well-defined. Mœglin showed that $r\left(w_{i},-\lambda_{i}, \Phi_{i}\right)$ is identically zero on $V^{\prime}\left(\mathfrak{p}_{i}\right)$ if $w_{i} \notin W(\uparrow$ , $\left.\mathfrak{p}_{i}\right)$. Since the local intertwining operators $R\left(w_{\mathfrak{p}}, \lambda_{\mathfrak{p}}\right)$ are well-defined by Proposition 3.1 , the only thing we need to show is that $r\left(d w_{1} \cdots w_{k} w_{0},-\lambda\right.$, $\left.\Phi_{D}\right)$ is holomorphic at $\lambda_{\mathfrak{p}}$ for $w_{i} \in W\left(\uparrow, \mathfrak{p}_{i}\right)$ even if $\chi=1$. Recall that for non-trivial $\chi, \chi \circ \alpha$ is non-trivial for $\alpha \in \Phi_{D}$ and so it is holomorphic. 
For this, we need to show that if for $w_{l} \in W\left(\uparrow, \mathfrak{p}_{l}\right), w_{l}\left(e_{r_{1}+\cdots+r_{l-1}+i}\right)<0$ for some $i=1, \ldots, r_{l}$, then $w_{l}\left(e_{r_{1}+\cdots+r_{l-1}+i}\right)=-e_{r_{1}+\cdots+r_{l}+1-i}$. Then the poles and zeros in $r\left(d w_{1} \cdots w_{k} w_{0},-\lambda, \Phi_{D}\right)$ cancel each other. We show it for $w_{1} \in W\left(\uparrow, \mathfrak{p}_{1}\right)$. The remaining cases are similar. Suppose $w_{1}\left(e_{i}\right)=-e_{j}$ for $1 \leq i, j \leq r_{1}$. Recall the definition of $W\left(\uparrow, \mathfrak{p}_{1}\right)$. It is the set of the Weyl group elements of $G_{1}^{\prime}$ which send $\left\{e_{i}-e_{j}, 1 \leq i<j \leq r_{1}\right\}$ to itself. Then consider $w_{1}\left(e_{p}-e_{i}\right)=w_{1}\left(e_{p}\right)+e_{j}$. So $w_{1}\left(e_{p}\right)=-e_{q}$, where $q>j$. Consider $w_{1}\left(e_{i}-e_{p}\right)=-e_{j}+w_{1}\left(e_{p}\right)$. Then $w_{1}\left(e_{p}\right)=-e_{q}$, where $q<j$. Therefore we can see that $w_{1}\left(e_{p}\right)=-e_{r_{1}+1-p}$.

Since $l_{\mathfrak{p}}\left(\phi, \lambda_{\mathfrak{p}}, \chi=1\right)$ is well-defined, by Mœglin's inner product formula (Section 2.2), it belongs to the residual spectrum $L^{2}(G(F) \backslash G(\mathbb{A}))_{(T, 1)}$. Its local components are precisely the image of intertwining operator $R\left(w_{\mathfrak{p}}, \lambda_{\mathfrak{p}}, \chi_{v}=1\right) I\left(\lambda_{\mathfrak{p}}, \chi_{v}=1\right)$. Even though Proposition 2.8 is no longer true since the normalized intertwining operators could vanish, we can show that the image of intertwining operator $R\left(w_{\mathfrak{p}}, \lambda_{\mathfrak{p}}, \chi_{v}=1\right) I\left(\lambda_{\mathfrak{p}}, \chi_{v}=1\right)$ is semi-simple in the same way as in Proposition 2.7. Then by Møglin [M1, p. 734], it is included in $\operatorname{Unip}(O)$, where $O$ is the unipotent orbit obtained by ignoring the ordering in $\mathfrak{p}$.

3.4. Parametrization of $\operatorname{Unip}(\mathfrak{p}, \chi)$. Next we parametrize $\operatorname{Unip}(\mathfrak{p}, \chi)$.

3.4.1. The case $\chi=1$. Because of Proposition 3.3.1, we can still define $R\left(\sigma_{\left(a_{i}, b_{i}\right)}\right)$ by Proposition 2.9 and the following still holds.

Proposition 3.4.1. $\sigma_{\left(a_{i}, b_{i}\right)} \longmapsto R\left(\sigma_{\left(a_{i}, b_{i}\right)}\right)$ is a homomorphism of the group $\left\{i d, \sigma_{\left(a_{i}, b_{i}\right)}\right\}$ into the group of the intertwining operators of $R\left(w_{\mathfrak{p}}, \lambda_{\mathfrak{p}}\right) I\left(\lambda_{\mathfrak{p}}\right)$.

This means the following: For $X \in \operatorname{Unip}(\mathfrak{p})$, let $R\left(\sigma_{\left(a_{i}, b_{i}\right)}\right) X=$ $\eta_{X}^{\mathfrak{p}}\left(\sigma_{\left(a_{i}, b_{i}\right)}\right) X$. Then $\eta_{X}^{\mathfrak{p}}$ defines a character of $A(O)$ such that $\eta_{X}^{\mathfrak{p}}\left(\sigma\left(a_{i}\right)\right)=$ $\eta_{X}^{\mathfrak{p}}\left(\sigma\left(b_{i}\right)\right)$.

Since $\operatorname{Unip}(\mathfrak{p}) \subset \operatorname{Unip}(O), \eta_{X}^{\mathfrak{p}} \in \operatorname{Springer}(O)$. Therefore we have:

Theorem 3.4.2. $\operatorname{Unip}(\mathfrak{p})$ is parametrized by

$$
\begin{aligned}
C(\mathfrak{p})=\{\eta \in \text { Springer }(O): & \eta\left(\sigma\left(a_{i}\right)\right)=\eta\left(\sigma\left(b_{i}\right)\right), \\
& \left.i=1, \ldots, s, \eta\left(\sigma\left(a_{s+1}\right)\right)=1\right\} .
\end{aligned}
$$

Example 3.4.1. Let $G=S p(8)$ and $\mathfrak{p}=(5,1,3)$. Then $\lambda_{\mathfrak{p}}=(2,1,0,1)$ and $w_{\mathfrak{p}}=c_{1} c_{2} c_{4} . O=(5,3,1)$ and Springer $(O)$ has 3 elements, namely, $\eta \in$ Springer $(O)$ if and only if $\eta(\sigma(5))=\eta(\sigma(3)), \eta(\sigma(1))=1$ or $\eta(\sigma(3))=$ $\eta(\sigma(1)), \eta(\sigma(5))=1$. Therefore, $\{\eta \in$ Springer $(O): \eta(\sigma(5))=\eta(\sigma(1))$, $\eta(\sigma(3))=1\}$ has only the trivial character.

In order to apply the above theorem to the global situation, let $O_{1}, O_{2}$ be two distinguished unipotent orbits in $G_{1}^{*}$ and $G_{2}^{*}$, resp. (If $G=S p(2 n)$, then $G_{1}^{*}=O\left(2 r_{1}, \mathbb{C}\right)$ and $G_{2}^{*}=O\left(2 r_{2}+1, \mathbb{C}\right)$. If $G=S O(2 n+1)$, then 
$G_{1}^{*}=S p\left(2 r_{1}, \mathbb{C}\right)$ and $G_{2}^{*}=S p\left(2 r_{2}, \mathbb{C}\right)$. If $G=O(2 n)$, then $G_{1}^{*}=O\left(2 r_{1}, \mathbb{C}\right)$ and $G_{2}^{*}=O\left(2 r_{2}, \mathbb{C}\right)$.) Then we get a unipotent orbit $O$ in $G^{*}$ by combining $O_{1}$ and $O_{2}$. Further we have canonical embedding $\widehat{A\left(O_{i}\right)} \subset \widehat{A(O)}$.

For $\mathfrak{p}_{i} \in P\left(O_{i}\right), i=1,2$, we get a chain $\mathfrak{p}_{1} \times \mathfrak{p}_{2}$ by shuffling the segments in $\mathfrak{p}_{1}$ and $\mathfrak{p}_{2}$ so that it satisfies (3.1), and thus we get $\operatorname{Unip}\left(\mathfrak{p}_{1} \times \mathfrak{p}_{2}\right)$. Let $\operatorname{Unip}\left(O_{1}, O_{2}\right)$ be the union of $\operatorname{Unip}\left(\mathfrak{p}_{1} \times \mathfrak{p}_{2}\right)$ as $\mathfrak{p}_{i}$ runs through $P\left(O_{i}\right)$ for $i=1,2$. It is a subset of $\operatorname{Unip}(O)$. Then we have:

Theorem 3.4.3. Unip $\left(O_{1}, O_{2}\right)$ is parametrized by

$$
\begin{aligned}
C\left(O_{1}, O_{2}\right)=\left\{\eta \in \text { Springer }(O):\left.\eta\right|_{A\left(O_{1}\right)} \in \operatorname{Springer}\left(O_{1}\right),\right. \\
\\
\left.\left.\eta\right|_{A\left(O_{2}\right)} \in \operatorname{Springer}\left(O_{2}\right)\right\} .
\end{aligned}
$$

This can be generalized easily. Let $O_{i}$ be distinguished unipotent orbits in $G_{i}^{*}$ for $i=0,1, \ldots, k$. Let $O$ be the unipotent orbit of $G^{*}$, obtained by combining $O_{i}$ 's. Then we can define $\operatorname{Unip}\left(O_{1}, \cdots, O_{k}, O_{0}\right)$ and it is a subset of $\operatorname{Unip}(O)$ and:

Theorem 3.4.4. Unip $\left(O_{1}, \cdots, O_{k}, O_{0}\right)$ is parametrized by

$$
\begin{aligned}
C\left(O_{1}, \ldots, O_{k}, O_{0}\right)=\left\{\eta \in \text { Springer }(O):\left.\eta\right|_{A\left(O_{i}\right)} \in \operatorname{Springer}\left(O_{i}\right),\right. \\
\text { for } i=0, \ldots, k\} .
\end{aligned}
$$

Corollary 3.4.5. Let $G=S p(2 n)$ and let $O_{1}=\left(q_{1}, \ldots, q_{s-1}, 1\right)$, s even, be a distinguished unipotent orbit of $O(2 n, \mathbb{C})$ and $O_{0}=(1)$. Then $\operatorname{Unip}\left(O_{1}, O_{0}\right)$ is parametrized by Springer $\left(O^{\prime}\right)$, where $O^{\prime}=\left(q_{1}, \ldots, q_{s-1}\right)$.

3.4.2. The case $\chi=\chi(\mu, \ldots, \mu), \mu$ non-trivial quadratic. Let $\mathfrak{p}=$ $\left(a_{1}, b_{1}, \ldots, a_{s}, b_{s}\right)$. In this case the above theorems for $\chi=1$ case hold. We need to use the generalized Iwahori-Matsumoto involution in Section 1. We can define $R\left(\sigma_{\left(a_{i}, b_{i}\right)}, \mu\right)$ in the similar way as in Proposition 2.9.

Let $\operatorname{Unip}(\mathfrak{p}, \mu)$ be the set of components of $R\left(w_{\mathfrak{p}}, \lambda_{\mathfrak{p}}, \chi\right) I\left(\lambda_{\mathfrak{p}}, \chi\right)$. Let $O$ be a unipotent orbit obtained from $\mathfrak{p}$ by ignoring the ordering. Then:

Theorem 3.4.6. $\operatorname{Unip}(\mathfrak{p}, \mu)$ is parametrized by

$$
C(\mathfrak{p})=\left\{\eta \in \text { Springer }(O): \eta\left(\sigma\left(a_{i}\right)\right)=\eta\left(\sigma\left(b_{i}\right)\right), i=1, \ldots, s\right\} .
$$

Let $O_{i}$ be distinguished unipotent orbits in $G_{i}^{*}$ for $i=1, \ldots, k$. Let $O$ be the unipotent orbit of $G^{*}$, obtained by combining $O_{i}$ 's. Then we can define $\operatorname{Unip}\left(O_{1}, \cdots, O_{k}, \mu\right)$ and:

Theorem 3.4.7. $\operatorname{Unip}\left(O_{1}, \cdots, O_{k}, \mu\right)$ is parametrized by

$$
\begin{aligned}
C\left(O_{1}, \ldots, O_{k}, \mu\right)=\left\{\eta \in \text { Springer }(O):\left.\eta\right|_{A\left(O_{i}\right)} \in \operatorname{Springer}\left(O_{i}\right),\right. \\
\text { for } i=1, \ldots, k\} .
\end{aligned}
$$


3.4.3. The general case. It is enough to consider the case, $\chi=$ $\chi(\underbrace{\mu, \ldots, \mu}_{r_{1}}, \underbrace{1, \ldots, 1}_{r_{0}})$, where $\mu$ is a non-trivial quadratic character. Let $\mathfrak{p}_{i}$, $i=1,2$, be chains in $G_{1}^{*}, G_{2}^{*}$, resp, from which we get unipotent orbits $O_{1}$, $\mathrm{O}_{2}$, by ignoring the ordering in $\mathfrak{p}_{i}$ 's. (If $G=S p(2 n)$, then $G_{1}^{*}=O\left(2 r_{1}, \mathbb{C}\right.$ ) and $G_{2}^{*}=O\left(2 r_{0}+1, \mathbb{C}\right)$. If $G=S O(2 n+1)$, then $G_{1}^{*}=S p\left(2 r_{1}, \mathbb{C}\right)$ and $G_{2}^{*}=S p\left(2 r_{0}, \mathbb{C}\right)$. If $G=O(2 n)$, then $G_{1}^{*}=O\left(2 r_{1}, \mathbb{C}\right)$ and $G_{2}^{*}=O\left(2 r_{0}, \mathbb{C}\right)$.) Let $\mathfrak{p}=\mathfrak{p}_{1} \times \mathfrak{p}_{2}$.

We obtain $R\left(\sigma_{i}\right)$ 's and $R\left(\sigma_{j}, \mu\right)$ 's. Let $\operatorname{Unip}(\mathfrak{p}, \chi)$ be the set of components of $R\left(w_{\mathfrak{p}}, \lambda_{\mathfrak{p}}, \chi\right) I\left(\lambda_{\mathfrak{p}}, \chi_{v}\right)$. Then:

Theorem 3.4.8. $\operatorname{Unip}(\mathfrak{p}, \chi)$ is parametrized by $C\left(\mathfrak{p}_{1}\right) \times C\left(\mathfrak{p}_{2}\right)$.

Let $\chi=\chi(\underbrace{\mu_{1}, \ldots, \mu_{1}}_{r_{1}}, \ldots, \underbrace{\mu_{k}, \ldots, \mu_{k}}_{r_{k}}, \underbrace{1, \ldots, 1}_{r_{0}}), r_{0}+\cdots+r_{k}=n, r_{1} \geq$ $\cdots \geq r_{k}, \mu_{i}$ 's are distinct non-trivial quadratic characters. Set $\mu_{0}=1$. Let $\mathfrak{p}_{i}, i=0,1, \ldots, k$, be chains in $G_{i}^{*}$, from which we get unipotent orbits $O_{1}, \ldots, O_{k}, O_{0}$, by ignoring the ordering in $\mathfrak{p}_{i}$ 's. Let $\mathfrak{p}=\mathfrak{p}_{1} \times \cdots \mathfrak{p}_{k} \times \mathfrak{p}_{0}$. Then:

Theorem 3.4.9. $\operatorname{Unip}(\mathfrak{p}, \chi)$ is parametrized by $C\left(\mathfrak{p}_{1}\right) \times \cdots \times C\left(\mathfrak{p}_{k}\right) \times C\left(\mathfrak{p}_{0}\right)$.

In order to apply the above theorem to the global situation, let $\chi=$ $\chi(\underbrace{\mu_{1}, \ldots, \mu_{1}}_{r_{1}}, \ldots, \underbrace{\mu_{k}, \ldots, \mu_{k}}_{r_{k}}, \underbrace{1, \ldots, 1}_{r_{0}}), r_{0}+\cdots+r_{k}=n, r_{1} \geq \cdots \geq r_{k} \geq$ $2, \mu_{i}$ 's are distinct non-trivial quadratic grössencharacters. Let $O_{i}$ be a distinguished unipotent orbit in $G_{i}^{*}$ for $i=0,1, \ldots, k$. Let $\mathfrak{p}_{i} \in P\left(O_{i}\right)$ for $i=0, \ldots, k$ and $\mathfrak{p}=\mathfrak{p}_{1} \times \cdots \times \mathfrak{p}_{k} \times \mathfrak{p}_{0}$. Then we can shuffle the segments in $\mathfrak{p}$ so that it satisfies the condition (3.1). We still call it $\mathfrak{p}$. For a nonarchimedean place $v$, let $\operatorname{Unip}\left(O_{1}, \ldots, O_{k}, O_{0}, \chi_{v}\right)$ be the set of union of $\operatorname{Unip}\left(\mathfrak{p}_{1}, \ldots, \mathfrak{p}_{k}, \mathfrak{p}_{0}, \chi_{v}\right)$ as $\mathfrak{p}_{i}$ runs through $P\left(O_{i}\right)$ for $i=0, \ldots, k$.

Theorem 3.4.10. $\Pi_{r e s_{v}}=\operatorname{Unip}\left(O_{1}, \ldots, O_{k}, O_{0}, \chi_{v}\right)$ is parametrized by

$$
\begin{aligned}
C\left(O_{1}, \ldots, O_{k}, O_{0}, \chi_{v}\right)=[ & \text { Springer }\left(O_{1}\right) \times \cdots \\
\times & \left.\operatorname{Springer}\left(O_{k}\right) \times \operatorname{Springer}\left(O_{0}\right)\right],
\end{aligned}
$$

where [ ] is defined as follows: If $\mu_{1 v}=\mu_{2 v} \neq \mu_{i v}$ for $i=0,3, \ldots, k$, then we replace Springer $\left(O_{1}\right) \times$ Springer $\left(O_{2}\right)$ by

$C\left(O_{1}, O_{2}, \mu_{1 v}\right)=\left\{\eta \in\right.$ Springer $(O):\left.\eta\right|_{A\left(O_{i}\right)} \in \operatorname{Springer}\left(O_{i}\right)$, for $\left.i=1,2\right\}$, where $O$ is the unipotent orbit of $G_{12}^{*}$ obtained by combining $O_{1}, O_{2}$, where

$$
G_{12}^{*}= \begin{cases}O\left(2\left(r_{1}+r_{2}\right), \mathbb{C}\right), & \text { if } G=S p(2 n), O(2 n) \\ S p\left(2\left(r_{1}+r_{2}\right), \mathbb{C}\right), & \text { if } G=S O(2 n+1) .\end{cases}
$$


Example 3.4.2. Let $G=S p(28)$ and $\chi=\chi(\underbrace{\mu, \ldots, \mu}_{10}, \underbrace{1, \ldots, 1}_{4}), \mu$ is a quadratic grössencharacter. Let $O_{1}=(9,7,3,1)$ be a unipotent orbit in $\mathrm{O}_{20}(\mathbb{C})$ and $\mathrm{O}_{2}=(5,3,1)$ a unipotent orbit in $O_{9}(\mathbb{C})$. Then for a nonarchimedean place $v$, if $\mu_{v} \neq 1$, then $\Pi_{r e s_{v}}$ is parametrized by Springer $\left(O_{1}\right) \times$ Springer $\left(\mathrm{O}_{2}\right)$. It has 18 elements. Let $\mu_{v}=1$. Let $O=(9,7,5,3,3,1,1)$. Then $A(O)$ is an abelian group generated by order 2 elements $\sigma(1), \sigma(3)$, $\sigma(5), \sigma(7), \sigma(9)$. Consequently

$$
\begin{aligned}
\text { Springer }(O)=\{\eta \in \widehat{A(O)}: \eta(\sigma(9)) & =\eta(\sigma(7)), \eta(\sigma(5))=1 \\
& \text { or } \eta(\sigma(7))=\eta(\sigma(5)), \eta(\sigma(9))=1\} .
\end{aligned}
$$

Then $C\left(O_{1}, O_{2}\right)=\left\{\eta \in\right.$ Springer $(O):\left.\eta\right|_{A\left(O_{i}\right)} \in$ Springer $\left.\left(O_{i}\right), i=1,2\right\}$. $C\left(O_{1}, O_{2}\right)$ has 5 elements.

\begin{tabular}{|c|c|c|c|c|c|}
\hline$\sigma(9)$ & 1 & -1 & 1 & 1 & -1 \\
\hline$\sigma(7)$ & 1 & -1 & -1 & 1 & -1 \\
\hline$\sigma(5)$ & 1 & 1 & -1 & 1 & 1 \\
\hline$\sigma(3)$ & 1 & 1 & -1 & -1 & -1 \\
\hline$\sigma(1)$ & 1 & 1 & 1 & -1 & -1 \\
\hline
\end{tabular}

Remark 3.4.1. Let $\lambda_{O}$ be the conjugate of $\lambda_{\mathfrak{p}}$ which is the closure of the positive Weyl chamber as in Lemma 3.2. Then by inducing in stages, $I\left(\lambda_{O}, w_{1}^{-1} \chi_{v}\right)=\operatorname{Ind}_{P}^{G} \lambda_{O} \otimes \operatorname{Ind}_{B}^{M} w_{1}^{-1} \chi_{v}$, where $P=M N$ is the parabolic subgroup such that $\lambda_{O}$ is in the positive Weyl chamber with respect to $P$. Then we can consider the Knapp-Stein $R$-group of $\operatorname{Ind}_{B}^{M} w_{1}^{-1} \chi_{v}$. It is a subset of (3.2). In fact, the Knapp-Stein $R$-group is spanned by the order 2 elements $c_{r_{1}+\cdots+r_{i}}$ for $\mu_{i v} \neq 1$. Therefore, we can think of (3.2) as a generalization of the Knapp-Stein $R$-group.

\section{References}

[A1] J. Arthur, On some problems suggested by the trace formula in 'Lie group representations', II, Lecture Notes in Math., 1041, Springer-Verlag, (1984), 1-49, MR 85k:11025, Zbl 541.22011.

[A2] Unipotent automorphic representations: conjectures, in 'Orbites unipotentes det représentations II. Groupes p-adiques et réels', Astérisque, 171-172 (1989), 13-71, MR 91f:22030, Zbl 728.22014.

[Au1] A.-M. Aubert, Dualité dans le groupe de Grothendieck de la catégorie des représentations lisses de longueur finie d'un groupe réductif p-adique, Trans. Amer. Math. Soc., 347 (1995), 2179-2189, MR 95i:22025, Zbl 827.22005. 
[Au2] _ Erratum à l'article "Dualité dans le groupe de Grothendieck de la catégorie des représentations lisses de longueur finie d'un groupe réductif padique”, Trans. Amer. Math. Soc., 348 (1996), 4687-4690, MR 97c:22019.

[B-Mo1] D. Barbasch and A. Moy, A unitarity criterion for p-adic groups, Inv. Math., 98 (1988), 19-37, MR 90m:22038, Zbl 676.22012.

[B-Mo2] __ Reduction to real infinitesimal character in affine Hecke algebras, J. of the AMS, 6(3) (1993), 611-635, MR 93k:22015.

[B-Z] I. Bernstein and A. Zelevinsky, Induced representations of reductive p-adic groups. I, Ann. Scient. Éc. Norm. Sup., 10 (1977) 441-472, Zbl 412.22015.

[Bo] A. Borel, Admissible representations of a semi-simple group over a local field with vectors fixed under an Iwahori subgroup, Invent. Math., 35 (1976), 233-259, MR 56 \#3196, Zbl 334.22012.

[Bu-K] C. Bushnell and P. Kutzko, Smooth representations of reductive p-adic groups: Structure theory via types, Proc. London Math. Soc., 77 (1998), 582-634, MR 2000c:22014, Zbl 911.22014.

[Ca] W. Casselman, Introduction to the theory of admissible representations of $p$-adic reductive groups, preprint.

[Ca2] The unramified principal series of p-adic groups I, Comp. Math., 40 (1980), 387-406, MR 83a:22018, Zbl 472.22004.

[G] D. Goldberg, Reducibility of induced representations for $S p(2 n)$ and $S O(n)$, Amer. J. Math., 116(5) (1994), 1101-1151, MR 95g:22016, Zbl 851.22021.

[Go] D. Goldstein, Heck algebra isomorphisms for tamely ramified characters, Ph.D. thesis, University of Chicago, 1990.

[I-M] N. Iwahori and H. Matsumoto, On some Bruhat decomposition and the structure of the Hecke rings of p-adic Chevalley groups, Publ. Math. IHES, 25 (1965), 5-48, MR 32 \#2486, Zbl 228.20015.

[Ja1] C. Jantzen Degenerate principal series for symplectic groups and odd-orthogonal groups, Mem. of AMS, 124(590) (1996), MR 97d:22020, Zbl 866.22016.

[Ja2] On the Iwahori-Matsumoto involution and applications, Ann. Scient. Éc. Norm. Sup., 28 (1995), 527-547, MR 97b:22020, Zbl 840.22030.

[Ja3] - On supports of induced representations for symplectic and odd-orthogonal groups, Amer. J. Math., 119 (1997), 1213-1262, MR 99b:22028, Zbl 888.22013.

[Ka-Lu] D. Kazhdan and G. Lusztig, Proof of the Deligne-Langlands conjecture for Hecke algebras, Invent. Math., 87 (1987), 153-215, MR 88d:11121, Zbl 613.22004.

[Ke-Sh] C.D. Keys and F. Shahidi, Artin L-functions and normalization of intertwining operators, Ann. Scient. Ec. Norm. Sup., 21 (1988), 67-89, MR 89k:22034, Zbl 654.10030.

[Ki1] H. Kim, The residual spectrum of $S p_{4}$, Comp. Math., 99(2) (1995), 129-151, MR 97c:11056, Zbl 877.11030.

[Ki2] The residual spectrum of $G_{2}$, Can. J. Math., 48(6) (1996), 1245-1272 MR 97i:11055, Zbl 879.11024.

[Ki3] _ Residual spectrum of split classical groups; contribution from Borel subgroups, Pacific J. Math., 199 (2001), 417-445. 
[Ki-Sh] H. Kim and F. Shahidi, Quadratic unipotent Arthur parameters and residual spectrum of $S p_{2 n}$, Amer. J. Math., 118(2) (1996), 401-425, MR 98f:11048, Zbl 866.11036.

[La1] R.P. Langlands, On the Functional Equations Satisfied by Eisenstein Series, Lecture Notes in Math., 544, Springer-Verlag, 1976, MR 58 \#28319, Zbl 332.10018.

[La2] - On the classification of irreducible representations of real algebraic groups, in 'Representation Theory and Harmonic Analysis on Semisimple Lie Groups', (1989), 101-170, MR 91e:22017, Zbl 741.22009.

[Lu1] G. Lusztig, Intersection cohomology complexes on a reductive group, Inv. Math., 75 (1984), 205-272, MR 86d:20050, Zbl 574.20032.

[Lu2] _ Representations of affine Hecke algebras, Astérisque, 171-172 (1989), 73-84, MR 90k:22028, Zbl 699.22027.

[M1] C. Mœglin, Représentations unipotentes et formes automorphes de carré intégrable, Forum Mathematicum, 6 (1994), 651-744, MR 95k:22024, Zbl 816.11034.

[M2] _ Orbites unipotentes et spectre discret non ramifie, Le cas des groupes classiques déployś, Comp. Math., 77 (1991), 1-54, MR 92d:11054, Zbl 809.11030.

[M3] Une conjecture sur le spectre résiduel des groupes classiques, preprint, 1994.

[M4] _ Représentations quadratiques unipotentes des groupes classiques padiques, Duke Math. J., 84 (1996), 267-332 MR 97h:22013, Zbl 864.22008.

[M5]_ Cuspidal quadratic unipotent representation, preprint, 1994.

[M6] Letters.

[M-W1] C. Møglin and J.L. Waldspurger, Spectral Decomposition and Eisenstein series, une paraphrase de l'Ecriture, Cambridge Tracts in Mathematics, 113, Cambridge University Press, 1995, MR 97d:11083, Zbl 846.11032.

[M-W2] _ Le spectre résiduel de $G L(n)$, Ann. Scient. Éc. Norm. Sup., 22 (1989), 605-674, MR 91b:22028, Zbl 696.10023.

[Mr] L. Morris, Tamely ramified intertwining algebras, Invent. Math., 114 (1993), 1-54, MR 94g:22035, Zbl 854.22022.

[Mu G. Muić, Some results on square integrable representations: Irreducibility of standard representations, IMRN, 14 (1998), 705-726, MR 99f:22031, Zbl 909.22029.

[Re1] M. Reeder, On certain Iwahori invariants in the unramified principal series, Pac. J. Math., 153 (1992), 313-342, MR 93b:22033, Zbl 804.22010.

[Re2] Nonstandard intertwining operators and the structure of unramified principal series representations, Forum Math., 9 (1997), 457-516, MR 98j:22028, Zbl 882.22020.

[R] A. Roche, Types and Hecke algebras for principal series representations of split reductive p-adic groups, Ann. Scient. Éc. Norm. Sup., 31 (1998), 361-413, MR 99d:22028, Zbl 903.22009.

[Sc-St] P. Schneider and U. Stuhler, Representation theory and sheaves on the Bruhat-Tits building, Publ. Math. IHES, 85 (1997), 97-191, MR 98m:22023, Zbl 892.22012.

[Sh1] F. Shahidi, Twisted endoscopy and reducibility of induced representations for padic groups, Duke Math. J., 66(1) (1992), 1-41, MR 93b:22034, Zbl 785.22022. 
[Sh2] - On the Ramanujan conjecture and finiteness of poles for certain Lfunctions, Ann. of Math., 127 (1988), 547-584, MR 89h:11021, Zbl 654.10029.

[Sp-V] B. Speh and D. Vogan, Reducibility of generalized principal series representations, Acta Math., 145 (1980), 227-299, MR 82c:22018, Zbl 457.22011.

[Ta1] M. Tadić, Representations of p-adic symplectic groups, Comp. Math., 90 (1994), 123-181, MR 95a:22025, Zbl 797.22008.

[Ta2] Structure arising from induction and Jacquet modules of representations of classical p-adic groups, J. of Algebra, 177 (1995), 1-33, MR 97b:22023, Zbl 874.22014.

[Ta3] - On regular square integrable representations of p-adic groups, Amer. J. Math., 120 (1998), 159-210, MR 99h:22026, Zbl 903.22008.

[Z] A. Zelevinsky, Induced representations of reductive p-adic groups II, On irreducible representations of $G L(n)$ Ann. Scient. Éc. Norm. Sup., 13 (1992), 165210, MR 83g:22012.

Received June 15, 1999. The second author was partially supported by NSF grant DMS9610387.

Dept. of Mathematics

Ohio State University

Columbus, $\mathrm{OH} 43210$

E-mail address: jantzen@math.ohio-state.edu

Dept. OF Mathematics

East Carolina University

Greenville, NC 27858

Dept. of Mathematics

SOUTHERN IllinoIS UNIVERSITY

Carbondale, IL 62901

E-mail address: henrykim@math.siu.edu 